\title{
Isomalto/malto-polysaccharides from starch \\ Reaction pathways and new characterization strategies
}

Pieter H. van der Zaal 


\section{Thesis committee}

\section{Promotor}

Prof. Dr J.H. Bitter

Professor of Biobased Chemistry \& Technology

Wageningen University \& Research

\section{Co-promotor}

Dr P.L. Buwalda

External staff member, Biobased Chemistry \& Technology

Wageningen University \& Research

Research Director at the AVEBE Food Innovation Center, Veendam

\section{Other members}

Prof. Dr W.J.H. van Berkel, Wageningen University \& Research

Dr A. Blennow, University of Copenhagen, Denmark

Prof. Dr G.J.W Euverink, University of Groningen

Dr M.A. Kabel, Wageningen University \& Research

This research was conducted under the auspices of the Graduate School VLAG (Advanced studies in Food Technology, Agrobiotechnology, Nutrition and Health Sciences). 


\section{Isomalto/malto-polysaccharides from starch \\ Reaction pathways and new characterization strategies}

\section{Pieter H. van der Zaal}

Thesis

submitted in fulfilment of the requirements for the degree of doctor

at Wageningen University

by the authority of the Rector Magnificus,

Prof. Dr A.P.J. Mol,

in the presence of the

Thesis Committee appointed by the Academic Board

to be defended in public

on Friday 14 December 2018

at 1:30 p.m. in the Aula. 
Pieter H. van der Zaal

Isomalto/malto-polysaccharides from starch - Reaction pathways and new characterization strategies, 134 pages.

$\mathrm{PhD}$ thesis, Wageningen University, Wageningen, the Netherlands (2018) With references, with summary in English

ISBN: 978-94-6343-371-6

DOI: $10.18174 / 463571$ 


\section{Table of contents}

$\begin{array}{lll}\text { Chapter } 1 & \text { General introduction } & 7\end{array}$

Chapter 2 Isomalto/malto-polysaccharide structure in relation to the 19 structural properties of starch substrates

Chapter 3 Enzymatic fingerprinting of isomalto/malto-polysaccharides

Chapter 4 The influence of linear substrates on 4,6- $\alpha$-glucanotransferase reaction dynamics during isomalto/malto-polysaccharide synthesis

Chapter 5 Directed modification of isomalto/malto-polysaccharides 

Chapter 1

General introduction 
Isomalto/malto-polysaccharides (IMMPs) have potential as a soluble food fibre (Leemhuis et al., 2014). These new fibres, IMMPs, can be made from one of the world's most abundant food sources, namely starch. It has been shown before (Leemhuis et al., 2014), that the 4,6- $\alpha$-glucanotransferase (GTFB) enzyme can modify starch in such a way that it becomes one of the desirable soluble food fibres. The enzyme cleaves glucose from the easily digestible $\alpha-(1 \rightarrow 4)$ glycosidic linkage and reattaches the glucose in a more difficult to digest $\alpha-(1 \rightarrow 6)$ linked glycosidic linkage (Bai et al., 2015; Dobruchowska et al., 2012; Leemhuis et al., 2014). Though the proof of principle has been given for several starches, the influence of the starch substrates' structural properties on the final IMMP structure and the role of additionally present sugars is currently unknown and the core topic of the research described in this thesis. The modification of starch with the GTFB enzyme results in new IMMPs which also need to be characterized. For that we have developed a new enzymatic fingerprinting method.

Before describing the results of the research, I will shortly discuss the relevance of (soluble) food fibres and the state-of-the art enzymatic modification of starch which can lead to the desired fibres.

\section{The fibre gap}

Globalization and urbanisation has resulted in an increased availability of foods rich in refined starch, sugar, salt and unhealthy fats (Hawkes, Harris, \& Gillespie, 2017). This, in combination with a widespread lack of physical activity and a generally sedentary lifestyle, has led to a sharp increase in non-communicable diseases (Kilpi et al., 2014). Non-communicable diseases such as type 2 diabetes, colorectal cancer and cardiovascular disease, are major public health problems that threaten the health and economies of all nations (Einarson, Acs, Ludwig, \& Panton, 2018). Although the causes of non-communicable diseases are complex, a large proportion of non-communicable diseases can be prevented by regular physical activity and maintaining a healthy diet (Nugent et al., 2018; World Health Organization, 2018). In order to avoid a global health crisis with major economic consequences (Einarson et al., 2018), it is important that the food industry works towards the production of healthy food products that are as enticing as their unhealthy counterparts. These products should not contribute to noncommunicable diseases and should be as available as their unhealthy counterparts.

Increased awareness of the link between non-communicable diseases and a healthy diet led already to an increased consumer demand for healthy foods, especially for products 
rich in food fibre (Stephen et al., 2017). Although the consumer is aware of the benefits of food fibres it often remains difficult to reach the recommended daily intake (Li \& Komarek, 2017). The difference between the recommended daily intake and the actual intake is often referred to as the 'fibre gap' (Zielinski et al., 2013). This is partly due to the poor organoleptic qualities and the lack of solubility of the most common food fibres, which makes them unattractive and difficult to use as a food additive (Li \& Komarek, 2017). Here lies a great opportunity for soluble food fibres, since they are easier to use as an additive and are less likely to cause organoleptic defects in the final product. These soluble fibres can be made from starch as has been shown by Leemhuis et al. (2014).

\section{Starch as starting point}

Starch is the most abundant food polysaccharide on earth, it is used for energy storage by green plants and consists purely of glucose molecules linked together in different ways. A small number of the starch's glucose monomers is present as $\alpha-(1 \rightarrow 4,6)$ linked glycosidic branching points, but the majority of starch's glucose monomers is linearly linked with $\alpha-(1 \rightarrow 4)$ glycosidic linkages (Figure 1).

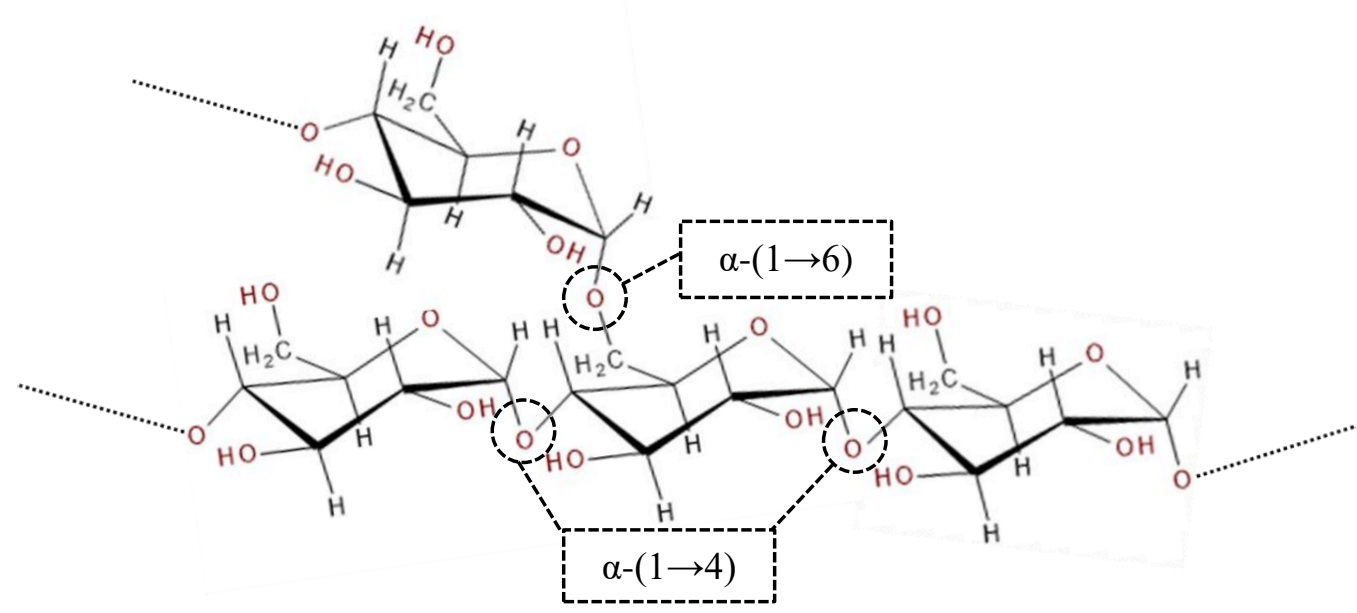

Figure 1. A depiction of an $\alpha-(1 \rightarrow 4,6)$ linked glycosidic branching point.

The $\alpha-(1 \rightarrow 4)$ - and $\alpha-(1 \rightarrow 6)$ linked glycosidic linkages are the basis for the two major starch components; amylopectin and amylose. Amylopectin is a branched polysaccharide that contains about $5 \%$ of $\alpha-(1 \rightarrow 4,6)$ linked glycosidic branching points and amylose is a primarily linear polysaccharide which consists mostly of linear $\alpha$ $(1 \rightarrow 4)$ linked glycosidic linkages (Figure 2) (Pérez \& Bertoft, 2010). Although amylose 
is a mostly linear polysaccharide, it can contain a small amount of $\alpha-(1 \rightarrow 4,6)$ linked glycosidic branching points (Takeda, Tomooka, \& Hizukuri, 1993). The amount of amylose is variable and ranges from being completely absent in the so-called 'waxy' starches to up to $80 \%$ in some amylomaize varieties (Table 1., McDonagh, 2012).

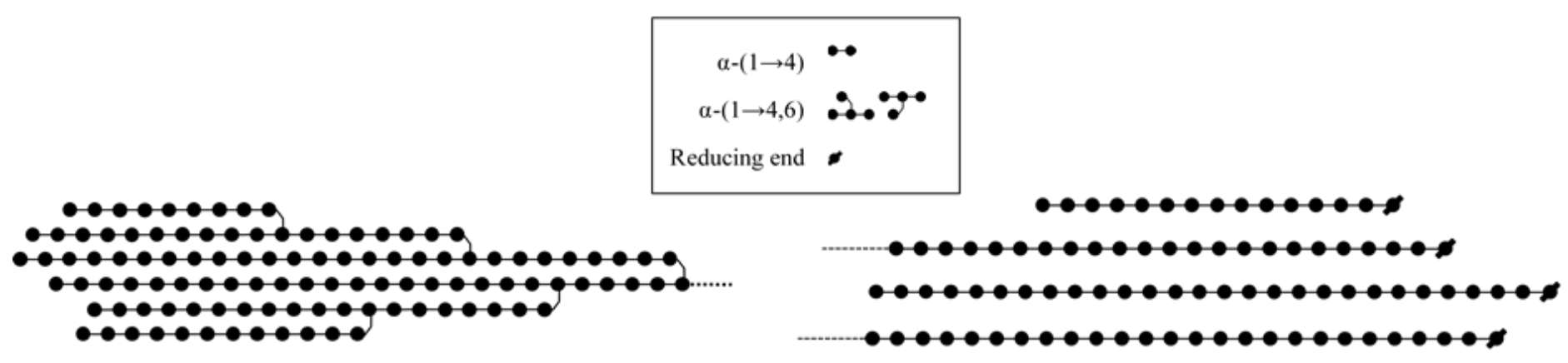

Figure 2. A simplified depiction of starch amylopectin (left) and starch amylose (right).

Table 1. Amylose content of several starches (Chen et al., 2003; Jane et al., 1999).

\begin{tabular}{|l|c|}
\hline Starch type & $\begin{array}{c}\text { Amylose } \\
\text { content } \\
\text { (apparent) }\end{array}$ \\
\hline Potato & 36 \\
Waxy potato & 0 \\
Maize & 29 \\
Waxy maize & 0 \\
Rice & 25 \\
Waxy rice & 0 \\
Sweet Potato & 19 \\
Wheat & 29 \\
Amylomaize V & 52 \\
Amylomaize VI & 68 \\
\hline
\end{tabular}


The linearity of subsequent $\alpha-(1 \rightarrow 4)$ linked glycosidic linkages, present in starch's amylopectin and amylose fractions, gives rise to the formation of single and double helices that are responsible for starch's unique techno-functional properties (Figure 3). The unique techno-functional properties of starch include starch crystallinity, retrogradation and its low solubility (Pérez \& Bertoft, 2010; Wang, Li, Copeland, Niu, \& Wang, 2015).

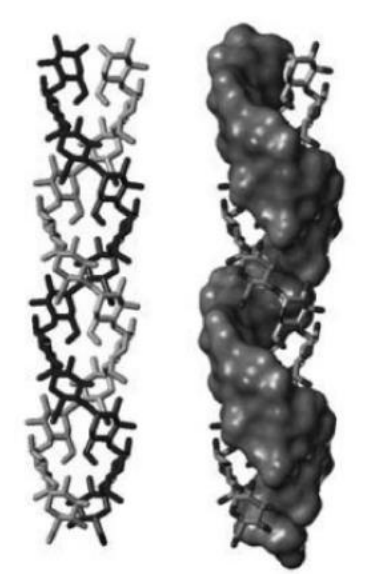

Figure 3. Schematic representation of a double helix structure commonly found in starch composed of two linear $\alpha-(1 \rightarrow 4)$ linked glycosidic chains, adapted from Pérez \& Bertoft (2010).

Even though starch only consists of glucose, there is still plenty of variety possible in starch such as granule size, amylose content and the degree of branching. Granule sizes, for example, can vary from $<1 \mu \mathrm{m}$ to $100 \mu \mathrm{m}$ and could either be homogenously or heterogeneously distributed, smooth or edged, depending on species and/or cultivar (Hoover, 2001; McDonagh, 2012; Pérez \& Bertoft, 2010). All the differences in starch are the result of the different enzymes that are active in the starch producing cultivar (Pérez \& Bertoft, 2010; Zhu, Bertoft, Szydlowski, d'Hulst, Christophe, \& Seetharaman, 2015). This natural variety of starch gives the possibility to select a desired property by selecting the right cultivar. However the options are limited since only a limited number of cultivars are readily available (for example containing a specific amount of amylose or a specific degree of branching). Therefore further modification, either enzymatic or chemical of the available starches is needed to make new products (Tomasik \& Schilling, 2004; van der Maarel \& Leemhuis, 2013). In that case the native properties of the starch will influence the properties of the final product. 


\section{Starch modifying enzymes}

Enzymes with the capability to modify starch can be divided into two distinct classes: glucanohydrolases and glucanotransferases. Glucanohydrolases are able to hydrolyse starch at different points of the starch molecule and glucanotransferases are able to transfer glucose from one starch molecule to another (van der Maarel et al., 2002). Both enzymes can show a little of the other activity since the enzymes are evolutionary quite close together. This depends mostly on substrate concentration as some glucanohydrolases are known to display transferase activity at high substrate concentrations (Kadokawa, 2011).

\section{Starch modification with glucanohydrolases}

Traditionally, most enzymatic modification of starch is done with glucanohydrolases such as $\alpha$-amylase, isoamylase, amyloglucosidase and $\beta$-amylase (van der Maarel et al., 2002). Depending on the glucanohydrolase type, the enzyme is able to cleave the glycosidic linkages of starch in different positions. Figure 4 shows a schematic representation of a starch molecule and displays the different possible points of hydrolysis depending on the type of glucanohydrolase that is used.

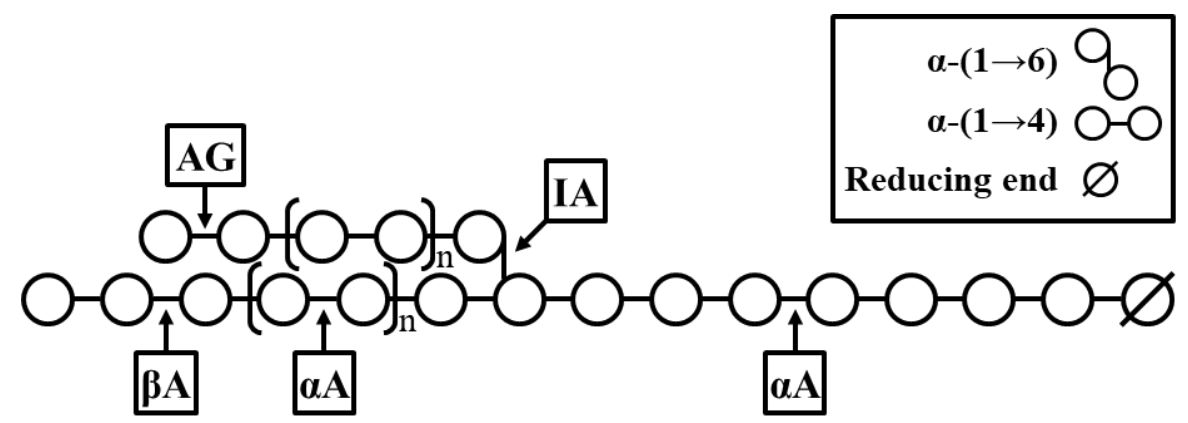

Figure 4. Schematic representation of the enzymatic activity of different glucanohydrolases on a starch molecule; $\alpha$-amylase $(\boldsymbol{\alpha A})$, isoamylase (IA), amyloglucosidase (AG) and $\beta$-amylase ( $\boldsymbol{\beta A})$.

The $\alpha$-amylase enzyme is endo-active, meaning that it is able to cleave $\alpha-(1 \rightarrow 4)$ linked glycosidic linkages in the middle of a starch molecule (Figure 4). $\alpha$-amylase activity is often accompanied with a significant drop in molecular weight, since hydrolysis with $\alpha$ amylase can occur in the middle of a starch molecule, easily halving its molecular weight with only a few hydrolytic cuts (van der Maarel et al., 2002). Isoamylase is also an endoactive enzyme, but the hydrolytic activity of isoamylase is limited to the $\alpha-(1 \rightarrow 6)$ 
glycosidic linkage of an $\alpha-(1 \rightarrow 4,6)$ glycosidic linked branching point. Isoamylase is often used to determine the chain length distribution (CLD) of branched $\alpha$-glucans such as starch amylopectin, since isoamylase only hydrolyses the branching points of its substrates (Jane, 1999). Amyloglucosidase and $\beta$-amylase are both exo-active enzymes meaning that they are only active on the 'outer' region of a starch substrate. Amyloglucosidase and $\beta$-amylase respectively cleave off glucose or maltose from the non-reducing end of starch's outer $\alpha-(1 \rightarrow 4)$ linked glycosidic chains. While starch modification with exo-active enzymes does not drastically decrease molecular weight it often results in big changes in techno-functional properties, since these exo-active enzymes are able to effectively 'shave' an amylopectin molecule (Leman, Goesaert, Vandeputte, Lagrain, \& Delcour, 2005).

\section{Glucanotransferases}

Glucanotransferases follow a slightly different mode of action compared to glucanohydrolases. While glucanohydrolases quickly hydrolyse the enzymeanhydroglucose intermediate with water, glucanotranferases are able to retain the enzyme-anhydroglucose intermediate by 'shielding' it from hydrolysis with water. This is due to the ability of glucanotransferases to create a greater affinity for the enzymeanhydroglucose intermediate to react with glycosidic acceptor molecules instead of water (Bissaro, Monsan, Fauré, \& O’Donohue, 2015). This means that glucanotransferases are able to modify the structure of $\alpha$-glucans, such as starch, by cleaving and reattaching anhydroglucose units. Glucanotransferases can create intriguing $\alpha$-glucan structures such as cyclo-amylose and $\alpha$-, $\beta$ - and $\gamma$-cyclodextrins, sometimes with surprising accuracy and repeatability (Crini, 2014). Another commercial example of a glucanotransferase enzyme is 4,4- $\alpha$-glucanotransferase, which creates a creates a disproportionation reaction between starch amylopectin and amylose, effectively elongating amylopectin chain length at the cost of the amylose fraction (Alting et al., 2009).

\section{4,6- $\alpha$-glucanotransferase}

The 4,6- $\alpha$-glucanotransferase (GTFB) enzyme that is used in this thesis displays a glucanotransferase activity that cleaves off an $\alpha-(1 \rightarrow 4)$ linked anhydroglucose unit from the non-reducing end and reattaches it in an $\alpha-(1 \rightarrow 6)$ linked position on the nonreducing end of a glycosidic acceptor molecule (Dobruchowska et al., 2012). The 
production and extraction of GTFB was optimized at the University of Groningen, methods were developed to measure the activity of GTFB and GTFB activity was studied on maltodextrins and model substrates such as amylose $\mathrm{V}$ and maltoheptaose (Bai, van der Kaaij, Leemhuis, et al., 2015; Bai, van der Kaaij, Woortman, Jin, \& Dijkhuizen, 2015). When the GTFB enzyme is used to modify starch substrates, it will have a transformative effect on the molecular structure and physicochemical properties of the produced isomalto/malto-polysaccharide, the change in this structure is exactly what we aim to investigate this thesis.

\section{The glucose puzzle}

The fact that the starch substrate and the isomalto/malto-polysaccharide product of the GTFB enzyme are both purely composed of glucose poses some challenges for analysis. Up till now, GTFB transferase activity has mostly been investigated with ${ }^{1} \mathrm{H}$ NMR spectroscopy, which was used to quantify the average amount of $\alpha-(1 \rightarrow 6)$ linked glycosidic linkages (Leemhuis et al., 2014). However, ${ }^{1} \mathrm{H}$ NMR spectroscopy cannot quantify the difference between starch's native $\alpha-(1 \rightarrow 4,6)$ linked glycosidic branching points and the linear $\alpha-(1 \rightarrow 6)$ linked glycosidic linkages introduced by the GTFB enzyme. ${ }^{1} \mathrm{H}$ NMR spectroscopy was used to identify the structure of some of the smaller isomalto/malto-oligosaccharide GTFB products (Dobruchowska et al., 2012). However, ${ }^{1} \mathrm{H}$ NMR spectroscopy cannot derive the same detailed structural information for larger polysaccharide structures. Therefore, to follow the complex reaction of starch with glucanotransferase type enzymes, we will need to develop a system of analysis that is able to pinpoint exactly where the starch molecules are modified. 


\section{Thesis aim and outline}

The aim of this research is to synthesize and characterize isomalto/maltopolysaccharides derived from the modification of starch with the GTFB enzyme and to find out how to control the production of these novel polysaccharides.

In chapter 2 we analyse the influence of the structural properties of starch-based substrates on the final IMMP structure. The produced isomalto/malto-polysaccharides were fractionated on a preparative scale using size exclusion chromatography (SEC-RI), the different fractions were the subsequently analysed with ${ }^{1} \mathrm{H}$ NMR, GPC-MALLS and methylation analysis. Methylation analysis was used to distinguish the native $\alpha-(1 \rightarrow 4,6)$ linked glycosidic branching points from the linear $\alpha-(1 \rightarrow 6)$ linked glycosidic linkages introduced by the GTFB enzyme. The extent of modification was investigated per molecular weight fraction in order to investigate the limits of GTFB modification on different substrate types.

In chapter 3 we present and demonstrate an enzymatic fingerprinting method specially developed for the analysis of isomalto/malto-polysaccharides. Isoamylase, isopullulanase, $\beta$-amylase and dextranase were selected on purity and selectivity and used separately, simultaneously or in successive order to structurally hydrolyse IMMPs. The enzymatic digests were then analysed with HPAEC and HPSEC chromatography to reveal the substructure of the produced IMMPs. This method was able to elucidate specific information on the substructure of IMMPs and pinpoint were the GTFB enzyme introduces its linear $\alpha-(1 \rightarrow 6)$ linked glycosidic linkages.

In chapter 4 we investigate the influence of chain length distribution on GTFB modification with two linear substrates which vary in chain length distribution. The linear substrates where produced by incubating waxy potato starch and amylomaltase modified potato starch with isoamylase. The reaction with GTFB was investigated over time and analysed with ${ }^{1} \mathrm{H}$ NMR, HPAEC and HPSEC chromatography. It was found that the GTFB enzyme shows more transferase activity in the presence of smaller glycosidic acceptors, such as mono and di-saccharides. The results obtained in this chapter were later used for the directed modification of IMMPs in chapter 5.

In chapter 5 we aim to control the modification of IMMPs towards different molecular weight products by the addition of a variety of mono/di-saccharide acceptors at different concentrations. The produced IMMPs were analysed with ${ }^{1} \mathrm{H}$ NMR, HPAEC, HPSEC and the amount of reducing ends was measured with a PAHBAH assay. It was found that the size of the final IMMP can be influenced by the type and concentration of added 
mono/di-saccharide acceptors, demonstrating that it is possible to control the outcome of a GTFB reaction.

In chapter 6 we discuss potential applications and the future perspectives of isomalto/malto-polysaccharides and the GTFB enzyme. 


\section{References}

Alting, A. C., van de Velde, Kanning, M. W., Burgering, M., Mulleners, L., Sein, A., ... Buwalda, P. (2009). Improved creaminess of low-fat yoghurt: The impact of amylomaltase-treated starch domains. Food Hydrocolloids, 23(3), 980-987. https://doi.org/10.1016/j.foodhyd.2008.07.011

Bai, Y., van der Kaaij, R. M., Leemhuis, H., Pijning, T., Leeuwen, S. S. van, Jin, Z., \& Dijkhuizen, L. (2015). Biochemical characterization of the Lactobacillus reuteri glycoside hydrolase family 70 GTFB type of 4,6- $\alpha$-glucanotransferase enzymes that synthesize soluble dietary starch fibers. Applied and Environmental Microbiology, 81(20), 7223-7232. https://doi.org/10.1128/AEM.01860-15

Bai, Y., van der Kaaij, R. M., Woortman, A. J. J., Jin, Z., \& Dijkhuizen, L. (2015). Characterization of the 4,6- $\alpha$-glucanotransferase GTFB enzyme of Lactobacillus reuteri 121 isolated from inclusion bodies. BMC Biotechnology, 15, 49. https://doi.org/10.1186/s12896-015-0163-7

Bissaro, B., Monsan, P., Fauré, R., \& O’Donohue, M. J. (2015). Glycosynthesis in a waterworld: new insight into the molecular basis of transglycosylation in retaining glycoside hydrolases. Biochemical Journal, 467(1), 17-35. https://doi.org/10.1042/BJ20141412

Chen, Z., Schols, H. ., \& Voragen, A. G. . (2003). Physicochemical Properties of Starches Obtained from Three Varieties of Chinese Sweet Potatoes. Food and Chemical Toxicology, 68(2), 431437. https://doi.org/10.1111/j.1365-2621.2003.tb05690.x

Crini, G. (2014). Review: A history of cyclodextrins. Chemical Reviews, 114(21), 10940-10975. https://doi.org/10.1021/cr500081p

Dobruchowska, J. M., Gerwig, G. J., Kralj, S., Grijpstra, P., Leemhuis, H., Dijkhuizen, L., \& Kamerling, J. P. (2012). Structural characterization of linear isomalto-/malto-oligomer products synthesized by the novel GTFB 4,6- $\alpha$-glucanotransferase enzyme from Lactobacillus reuteri 121. Glycobiology, 22(4), 517-528. https://doi.org/10.1093/glycob/cwr167

Einarson, T. R., Acs, A., Ludwig, C., \& Panton, U. H. (2018). The Economic Burden of Cardiovascular Disease in Type 2 Diabetes: A Systematic Review. Value in Health, 21(7), 881890. https://doi.org/10.1016/j.jval.2017.12.019

Hawkes, C., Harris, J., \& Gillespie, S. (2017). Chapter 4: Urbanization and the nutrition transition. In 2017 Global food policy report (pp. 34-41). Washington, DC: International Food Policy Research Institute (IFPRI). https://doi.org/10.2499/9780896292529

Hoover, R. (2001). Composition, molecular structure, and physicochemical properties of tuber and root starches: A review. Carbohydrate Polymers, 45(3), 253-267. https://doi.org/10.1016/S01448617(00)00260-5

Jane, J. (1999). Effects of amylopectin branch chain-length and amylose content on the gelatinization and pasting properties of starch. Cereal Chem., 52(3258), 555.

https://doi.org/10.1094/CCHEM.1999.76.5.629

Kadokawa, J. I. (2011). Precision Polysaccharide Synthesis Catalyzed by Enzymes. Chemical Reviews, 111(7), 4308-4345. https://doi.org/10.1021/cr100285v

Kilpi, F., Webber, L., Musaigner, A., Aitsi-Selmi, A., Marsh, T., Rtveladze, K., ... Brown, M. (2014). Alarming predictions for obesity and non-communicable diseases in the Middle East. Public Health Nutrition, 17(5), 1078-1086. https://doi.org/10.1017/S1368980013000840

Leemhuis, H., Dobruchowska, J. M., Ebbelaar, M., Faber, F., Buwalda, P. L., Van Der Maarel, M. J. E. C., ... Dijkhuizen, L. (2014). Isomalto/malto-polysaccharide, a novel soluble dietary fiber 
made via enzymatic conversion of starch. Journal of Agricultural and Food Chemistry, 62(49), 12034-12044. https://doi.org/10.1021/jf503970a

Leman, P., Goesaert, H., Vandeputte, G. E., Lagrain, B., \& Delcour, J. A. (2005). Maltogenic amylase has a non-typical impact on the molecular and rheological properties of starch. Carbohydrate Polymers, 62(3), 205-213. https://doi.org/10.1016/j.carbpol.2005.02.023

Li, Y. O., \& Komarek, A. R. (2017). Dietary fibre basics: Health, nutrition, analysis, and applications. Food Quality and Safety, 1(1), 47-59. https://doi.org/10.1093/fqs/fyx007

McDonagh, P. (2012). Native, modified and clean label starches in foods and beverages. Natural Food Additives, Ingredients and Flavourings. Woodhead Publishing Limited. https://doi.org/10.1016/B978-1-84569-811-9.50007-4

Nugent, R., Bertram, M. Y., Jan, S., Niessen, L. W., Sassi, F., Jamison, D. T., ... Beaglehole, R. (2018). Investing in non-communicable disease prevention and management to advance the Sustainable Development Goals. The Lancet, 391(10134), 2029-2035. https://doi.org/10.1016/S0140-6736(18)30667-6

Pérez, S., \& Bertoft, E. (2010). The molecular structures of starch components and their contribution to the architecture of starch granules: A comprehensive review. Starch - Stärke, 62. https://doi.org/10.1002/star.201000013

Stephen, A. M., Champ, M. M. J., Cloran, S. J., Fleith, M., Van Lieshout, L., Mejborn, H., \& Burley, V. J. (2017). Dietary fibre in Europe: Current state of knowledge on definitions, sources, recommendations, intakes and relationships to health. Nutrition Research Reviews (Vol. 30). https://doi.org/10.1017/S095442241700004X

Takeda, Y., Tomooka, S., \& Hizukuri, S. (1993). Structures of branched and linear molecules of rice amylose. Carbohydrate Research, 246(1), 267-272. https://doi.org/10.1016/00086215(93)84038-8

Tomasik, P., \& Schilling, C. H. (2004). Chemical modification of starch. Adv. Carbohydr. Chem. Biochem., 59(04), 175-403. https://doi.org/10.1016/s0065-2318(04)59005-4

van der Maarel, M., \& Leemhuis, H. (2013). Starch modification with microbial $\alpha$-glucanotransferase enzymes. Carbohydrate Polymers, 93(1), 116-121. https://doi.org/10.1016/j.carbpol.2012.01.065

van der Maarel, M., van der Veen, B., Uitdehaag, J., Leemhuis, H., \& Dijkhuizen, L. (2002). Properties and applications of starch-converting enzymes of the $\alpha$-amylase family. Journal of Biotechnology, 94(2), 137-155. https://doi.org/10.1016/S0168-1656(01)00407-2

Wang, S., Li, C., Copeland, L., Niu, Q., \& Wang, S. (2015). Starch Retrogradation: A Comprehensive Review, 14(5), 568-585. https://doi.org/10.1111/1541-4337.12143

World Health Organization. (2018). Global action plan on physical activity 2018-2030: more active people for a healthier world.

Zhu, F., Bertoft, E., Szydlowski, N., d'Hulst, Christophe, \& Seetharaman, K. (2015). Branching patterns in leaf starches from Arabidopsis mutants deficient in diverse starch synthases. Carbohydr. Res., 401, 96-108. https://doi.org/10.1016/j.carres.2014.09.011

Zielinski, G., DeVries, J. W., Craig, S. A., Bridges, A. R., Bridges, A., Vyth, E., ... Latulippe, M. (2013). Dietary Fiber Methods in Codex Alimentarius : Current Status and Ongoing Discussions. Cereal Foods World, 58(3), 148-152. https://doi.org/10.1094/CFW-58-3-0401 


\section{Chapter 2}

\section{Isomalto/malto-polysaccharide structure in relation to the structural properties of starch substrates}

Isomalto/malto-polysaccharides (IMMPs) are soluble dietary fibres produced by the enzymatic modification of starch with 4,6- $\alpha$-glucanotransferase (GTFB). The structure, size, and linkage distribution of these IMMPs has remained largely unknown, since most structural information has been based on indirect measurements such as total $\alpha-(1 \rightarrow 6)$ content, iodine staining and GTFB hydrolytic activity. This study provides a deeper understanding of IMMP structure in relation to its respective starch substrate, by combining preparative fractionation with linkage composition analysis. IMMPs were produced from a variety of amylose-rich and amylose-free starches. The extent of modification was investigated per IMMP molecular weight (Mw)-fraction, distinguishing between linear $\alpha-(1 \rightarrow 6)$ linkages introduced by GTFB and starch's native $\alpha-(1 \rightarrow 4,6)$ branching points. It emerged that the amount of $\alpha-(1 \rightarrow 6)$ linkages was consistently higher in IMMP low Mw-fractions and that GTFB activity was limited by native $\alpha-(1 \rightarrow 4,6)$ linkages. The presence of amylose turned out to be a prerequisite for the incorporation of linear $\alpha-(1 \rightarrow 6)$ linkages in amylopectin.

Based on: van der Zaal, P.H., Schols, H. A., Bitter J. H. \& Buwalda, P. L. (2017). Isomalto/malto-polysaccharide structure in relation to the structural properties of starch substrates. Carbohydrate Polymers. https://doi.org/10.1016/j.carbpol.2017.11.072 


\section{Introduction}

Starch is one of the most used polysaccharides in both food and non-food applications because of its broad functionality. The functionality of starch is the result of its molecular structure, which depends on aspects such as; amylose content, degree of branching and amylopectin chain length (Pérez \& Bertoft, 2010). These aspects vary per starch source and thus, different starches are used for different applications. While starch is being used on a large scale, its unmodified form is not suited for all applications.

In order to further increase the functionality of starch, it is often modified. Most starches are further functionalized with post-harvest modification, since it is impractical to rely solely on starch origin variety. Post-harvest modification of starch is traditionally done chemically using processes such as; hydrolysis, dextrinization, cross-linking or the addition of functional groups (Tomasik \& Schilling, 2004). Enzymes can also be used for the post-harvest modification of starch (Kadokawa, 2011; van der Maarel \& Leemhuis, 2013). The advantages of enzymatic over chemical modification of starch include; the absence of harsh chemicals, lower energy input and an even more selective modification. Therefore enzymes are an increasingly interesting tool for the post-harvest modification of starch (van der Maarel \& Leemhuis, 2013).

Enzymatic modification of starch is traditionally focussed on controlled breakdown of glucan chains using glucanohydrolases (Guzmán-Maldonado \& Paredes-López, 1995; van der Maarel et al., 2002). Commonly used glucanohydrolases in this field are; $\alpha$ amylase, maltogenic amylase (Leman et al., 2005), $\beta$-amylase and iso-amylase (Ciric et al., 2014). Next to glucanohydrolases, glucanotransferases can also be used for the modification of starch. Glucanotransferases are capable of modifying starch and other $\alpha$-glucans by altering the intrinsic linkage composition, by for example changing $\alpha$ $(1 \rightarrow 4)$ glycosidic linkages into $\alpha-(1 \rightarrow 6)$ glycosidic linkages (Kralj et al., 2011). Well documented glucanotransferases include; cyclodextrin glucanotransferase (Bissaro et al., 2015; Crini, 2014), 4,4- $\alpha$-glucanotransferase (Ayudhaya et al. 2016; van der Maarel \& Leemhuis, 2013; Xu et al., 2014) and branching enzymes (Grimaud et al., 2013; Suzuki et al., 2015).

Innovative $\alpha$-glucan structures can be produced by modifying starch with a combination of glucanohydrolases and glucanotransferases. The resulting $\alpha$-glucan is a combination of its natural structure and structural elements introduced by the action of the enzymes. Examples of $\alpha$-glucans produced with synergistic enzyme action are cyclo-isomaltooligosaccharides (Funane et al., 2014), enzymatically synthesized glycogen (Kajiura et al., 2010), isomalto-oligosaccharides (IMOs) (Kaulpiboon et al., 2015), highly branched 
maltodextrins (Lee et al., 2013) and the alternating elongation and branching of amylopectin using amylomaltase and branching enzymes (Sorndech et al., 2015). As shown above, a wide array of structures can be obtained with the enzymatic modification of $\alpha$-glucans. In this paper we investigate the unexplored structure of starches modified with the 4,6- $\alpha$-glucanotransferase (GTFB) enzyme, an enzyme that is capable of converting $\alpha-(1 \rightarrow 4)$ glycosidic linkages into $\alpha-(1 \rightarrow 6)$ glycosidic linkages.

The modification of starch with the GTFB enzyme results in the formation of isomalto/malto-polysaccharides (IMMPs). IMMPs are not to be confused with IMOs covering a DP range from 2 to $\sim 10$ (Chockchaisawasdee \& Poosaran, 2013; Goffin et al., 2011; Hu et al. 2013; Kaulpiboon et al., 2015) since IMMPs are considerably larger, IMMPs up to DP 35 have already been identified (Leemhuis et al., 2014). The functionality of most novel $\alpha$-glucans is directed towards slow digestibility, prebiotic functionality and application as a dietary fibre, this is usually done by increasing the amount of branching points, increasing crystallinity or by chemical modification (Lee et al., 2013; Raigond et al. 2015). The GTFB enzyme is able to decrease the digestibility of starch by reducing the amount of easily digestible $\alpha-(1 \rightarrow 4)$ linkages and introducing linear $\alpha-(1 \rightarrow 6)$ linkages that are not degradable by $\alpha$-amylase. Therefore, IMMPs have potential applications in food as slow-digestible fibres with prebiotic potential (Dijkhuizen et al., 2010; Leemhuis et al., 2014).

The activity of GTFB (Bai et al., 2015a), its crystal structure (Bai et al., 2016a) and action on different substrates (Bai et al., 2016b; Dobruchowska et al., 2012; Leemhuis et al., 2014) has been studied. To date, it has been demonstrated that GTFB is able to partially convert starch to IMMPs and it was proposed that the extent of GTFB modification is related to the amount of amylose in the substrate (Leemhuis et al., 2014). Although some research has been carried out on IMMP structure, most structural information on IMMPs so far, is based on indirect measurements such as total $\alpha-(1 \rightarrow 6)$ content, iodine staining and GTFB hydrolytic activity. Until now, the structure, size and linkage distribution of starch-based IMMPs has remained largely unknown.

This study is the first to fractionate starch-based IMMPs, and the first to differentiate between starch's native $\alpha-(1 \rightarrow 4,6)$ branching points and the linear $\alpha-(1 \rightarrow 6)$ linkages introduced by the GTFB- $\Delta \mathrm{N}$ enzyme, instead of solely relying on the total $\alpha-(1 \rightarrow 6)$ content measured with ${ }^{1} \mathrm{H}$ NMR spectroscopy. IMMPs were produced from a selection of starches and subsequently fractionated on a preparative scale. The linkage content was analysed with ${ }^{1} \mathrm{H}$ NMR and permethylation analysis, the molecular weight was determined with GPC-MALLS. Combining fractionation with linkage compositition 
analysis makes it possible to determine the extent of GTFB- $\Delta$ N modification in relation to the molecular weight of the IMMP fractions. This in-depth characterization also provides more information on the relation between the GTFB- $\Delta$ N reaction pathway and the molecular structure of the starch substrate. 


\section{Materials \& methods}

\subsection{Materials}

Potato starch, waxy potato starch (Eliane 100) and wheat starch (Excelsior) were provided by AVEBE (Veendam, the Netherlands). Maize starch (C-Gel) (Cargill, Wayzata, MN, USA) waxy maize starch (Amioca power TF, National Starch), rice starch (S7260, Sigma-Aldrich, St. Louis, MO, USA) and waxy rice starch (Remyline XS, Beneo, Mannheim, Germany) were purchased from their respective supplier. Sweet potato starch (SuShu2) was provided by the laboratory of Food Chemistry, Wageningen University \& Research (Wageningen, the Netherlands ) (Zhao et al., 2015).

\subsection{Production of GTFB- $\triangle N$}

The GTFB- $\Delta \mathrm{N}$ enzyme was produced in cooperation with Dr. Y. Bai, who kindly provided the E. coli BL21 DE3 cells carrying the pET15b- $\triangle$ NGTFB plasmid as described and produced in Bai et al. (2015b). The E. coli cells were grown at $37{ }^{\circ} \mathrm{C}$ for $16 \mathrm{~h}$ shaking at $220 \mathrm{rpm}$ in flasks containing LB medium supplemented with $100 \mu \mathrm{g} / \mathrm{mL}$ ampicillin. The culture was transferred into $600 \mathrm{~mL}$ flasks and kept at $37{ }^{\circ} \mathrm{C}$ for $2-3 \mathrm{~h}$ at $220 \mathrm{rpm}$ until $\mathrm{OD}_{600}=0.4$. The flasks were cooled on ice, $0.1 \mathrm{mM}$ isopropyl $\beta$-D-1thiogalactopyranoside was added and the flasks were incubated at $18{ }^{\circ} \mathrm{C}$ for $22 \mathrm{~h}$ at 160 $\mathrm{rpm}$. Cells were centrifuged at $4000 \mathrm{x} g$ at $10^{\circ} \mathrm{C}$ for $30 \mathrm{~min}$. The pellets were washed with washing buffer (20 mM Tris- $\mathrm{HCl}, \mathrm{pH}=8.0)$ and centrifuged at $3000 \mathrm{x} g$ at $10{ }^{\circ} \mathrm{C}$ for $30 \mathrm{~min}$. Each pellet was suspended in $15 \mathrm{~mL}$ lysis buffer $(20 \mathrm{mM}$ Tris- $\mathrm{HCl}, \mathrm{pH}=8.0$, $250 \mathrm{mM} \mathrm{NaCl}, 1 \mathrm{mM} \mathrm{CaCl}$ ), $100 \mu \mathrm{L}$ Lysozyme $(50 \mathrm{mg} / \mathrm{mL})$ and $20 \mu \mathrm{L}$ DNase (20 $\mathrm{mg} / \mathrm{mL}$ ) was added and left to rotate at room temperature for $2 \mathrm{~h}$. The suspension was lysed three times with a French press and centrifuged at $10,000 \mathrm{x} g$ at $10^{\circ} \mathrm{C}$ for $30 \mathrm{~min}$. The supernatant was retained and mixed with Ni-NTA beads (Sigma-Aldrich, St. Louis, MO, USA) at $4{ }^{\circ} \mathrm{C}$ overnight, GTFB- $\Delta \mathrm{N}$ was then purified using His-tag affinity column chromatography. After washing steps high purity GTFB- $\Delta$ N was eluted with a $300 \mathrm{mM}$ imidazole elution buffer. The final GTFB- $\Delta \mathrm{N}$ concentration was determined using a Nanodrop 2000 spectrophotometer (Isogen Life Science, De Meern, the Netherlands).

\subsection{GTFB- $\triangle N$ activity}

GTFB- $\Delta$ N hydrolytic activity on maltoheptaose (Sigma-Aldrich, St. Louis, MO, USA) was measured with a GOPOD assay (Megazyme, Bray, Wicklow, Ireland). The hydrolytic activity of the GTFB- $\Delta$ N enzyme used in this paper is comparable to previous research (Bai et al., 2015a). 


\subsection{IMMP synthesis}

Starch substrate was suspended at $2.5 \%(\mathrm{w} / \mathrm{v})$ in $20 \mathrm{mM}$ acetate buffer, $\mathrm{pH}=4.9$ containing $5 \mathrm{mM} \mathrm{CaCl}_{2}$. The starch suspension was gelatinized by autoclaving at 121 ${ }^{\circ} \mathrm{C}$ for $15 \mathrm{~min}$. GTFB- $\Delta \mathrm{N}$ was added after this sterilization step as soon as the reaction mixture was cooled down to $37{ }^{\circ} \mathrm{C}$, to avoid excessive retrogradation. IMMP synthesis was carried out by adding $0.3 \mathrm{mg}$ GTFB- $\Delta \mathrm{N} / \mathrm{g}$ substrate and incubating the solution at $37^{\circ} \mathrm{C}$ for $24 \mathrm{~h}$. Possible acidification was monitored by checking the $\mathrm{pH}$ before and after modification. The $\mathrm{pH}$ increased by an average of 0.1 in each sample, which indicates that the samples were not contaminated. After reaction, GTFB- $\Delta \mathrm{N}$ was inactivated by heating the reaction mixture to $95{ }^{\circ} \mathrm{C}$ for $15 \mathrm{~min}$ in a water bath. Next, the solution was cooled to $50^{\circ} \mathrm{C}$, Amberlite MB 20-resin (DOW, Midland, MI, USA) was added and the mixture was incubated at $50{ }^{\circ} \mathrm{C}$ for $2 \mathrm{~h}$. The MB20-resin was sieved out. The IMMP solution was stored at $-20{ }^{\circ} \mathrm{C}$ overnight and subsequently freeze-dried. IMMP yield (w/w) was determined by comparing the freeze-dried IMMP weight to the amount of starch substrate used, supplementary information (7.1).

\subsection{Fractionation with Size Exclusion Chromatography}

Preparative fractionation was executed on an Akta Explorer (GE Healthcare, Uppsala, Sweden) with a Sephacryl S-500, $4.3 \mathrm{~L}(\mathrm{r}=5 \mathrm{~cm}, \mathrm{~h}=55 \mathrm{~cm})$ BPG column (GE Healthcare, Uppsala, Sweden), using milliQ as an eluent. The IMMP samples were dissolved in milliQ at a concentration of $5 \mathrm{mg} / \mathrm{mL}$. $80 \mathrm{~mL}$ of this solution was applied on to the $\mathrm{S}$ 500 column. Three fractions were manually collected in accordance with the RI-signal; a high molecular weight (HMW)-, a medium molecular weight (MMW)- and a low molecular weight (LMW)-fraction. The collected fractions were concentrated with a rotary evaporator, stored at $-20{ }^{\circ} \mathrm{C}$ overnight and subsequently freeze-dried. Fractionation yield $(\mathrm{w} / \mathrm{w})$ was determined by comparing the cumulative freeze-dried IMMP HMW, MMW and LMW fractions to the injected amount of respective IMMP, supplementary information (7.3).

\subsection{Free glucose determination}

Free glucose was measured before and after IMMP synthesis with the GOPOD assay (Megazyme, Bray, Wicklow, Ireland). The deactivated sample $(100 \mu \mathrm{L})$ was mixed with $3 \mathrm{~mL}$ of GOPOD reagents, incubated at $50{ }^{\circ} \mathrm{C}$ for 20 minutes and the absorbance at 510 nm was measured with a DU 720 UV/vis spectrophotometer (Beckman Coulter, Brea, CA, USA). 


\section{$2.7^{1} H$ NMR Spectroscopy}

The total $\alpha-(1 \rightarrow 6)$ content was measured with ${ }^{1} \mathrm{H}$ NMR spectroscopy. Freeze-dried IMMP was exchanged once with $\mathrm{D}_{2} \mathrm{O}$ by lyophilisation and dissolved in $\mathrm{D}_{2} \mathrm{O}$ (99.9 atom $\%$ D, Sigma-Aldrich, St. Louis, MO, USA) at a concentration of $5 \mathrm{mg} / \mathrm{ml}$. Samples were shaken and heated up to $340 \mathrm{~K}$ in a Thermomixer (Eppendorf, Hamburg, Germany) to ensure maximal solubility during the NMR measurement. 1D ${ }^{1} \mathrm{H}$ NMR spectra were recorded at $340 \mathrm{~K}$ on a Bruker (Billerica, MA, USA) Avance 500 spectrometer or a Bruker Avance 600 spectrometer equipped with a cryo-probe. Both spectrometers are located at the Wageningen NMR Centre. The total $\alpha-(1 \rightarrow 6)$ content was determined by dividing the peak surface area at $5.0 \mathrm{ppm}(\alpha-(1 \rightarrow 6))$ by the peak surface areas at 5.0 and $5.4 \mathrm{ppm}(\alpha-(1 \rightarrow 4))$. If there was an overlap between the peak surface areas at $5.4 \mathrm{ppm}$ and $5.2 \mathrm{ppm}$ ( $\alpha$-reducing end), the peak surface area of the $\alpha$-reducing end was subtracted from the $\alpha-(1 \rightarrow 4)$ peak surface area. Structures were characterized using the ${ }^{1} \mathrm{H}$ NMR structural-reporter-group concept for $\alpha$-D-glucans (Dobruchowska et al., 2012; van Leeuwen et al., 2008).

\subsection{Linkage composition analysis}

The $\alpha-(1 \rightarrow 6)$ and $\alpha-(1 \rightarrow 4,6)$ content of IMMPs and their respective fractions were determined with permethylation analysis. Permethylation was performed as described in the protocol of (Pettolino et al., 2012). Free hydroxyl groups in the IMMP samples were methylated with methyl iodide, the samples were then hydrolysed with trifluoroacetic acid, reduced with sodium borodeuteride and subsequently acetylated with acetic anhydride into partially methylated alditol acetates (PMAAs). The produced PMAAs were dissolved in ethyl acetate. Product identification and quantification was done by GC-MS running a temperature gradient from $120{ }^{\circ} \mathrm{C}$ to $250{ }^{\circ} \mathrm{C}$ in 52 minutes and remaining constant at $250^{\circ} \mathrm{C}$ for 5 minutes. The GC-MS system consists of a Trace GC Ultra GC (Thermo Fisher Scientific, Waltham, MA, USA) with a Rtx-35MS (Restek Corporation, Bellefonte, PA, USA) column (30m, internal diameter $0.25 \mathrm{~mm}$ ) and a DSQII MS (Thermo Fisher Scientific) using positive mode, $\mathrm{m} / \mathrm{z}$ range 50-450. Data were processed using Xcalibur 2.2 software (Thermo Fisher Scientific).

\subsection{GPC-MALLS}

Samples were dissolved in DMSO- $\mathrm{LiBr}(0.05 \mathrm{M})$ to achieve a concentration of $2 \mathrm{mg} / \mathrm{mL}$ and heated to $80^{\circ} \mathrm{C}$ for 30 minutes. The cooled samples were filtered through a $0.45 \mu \mathrm{m}$ PTFE membrane (Pall Life Sciences, Ann Arbor, MI, USA). The samples were then injected in a GPC-MALLS system (Agilent Technologies 1260 Infinity) from PSS 
(Mainz, Germany) with isocratic pump, auto sampler, online degasser, inline $0.2 \mu \mathrm{m}$ filter, RI detector (G1362A 1260 RID Agilent Technologies, viscometer (ETA-2010 PSS, Mainz), MALLS detector (SLD 7000 PSS, Mainz). DMSO-LiBr (0.05 M) was used as eluent. The samples were injected with a flow rate of $0.5 \mathrm{ml} \mathrm{min}^{-1}$ into three PFG SEC columns 100, 300, and 4000, purchased from PSS. The columns were held at $80{ }^{\circ} \mathrm{C}$, and the detectors were held at $60{ }^{\circ} \mathrm{C}$ (Visco) and $45^{\circ} \mathrm{C}$ (RI) respectively. A pullulan kit (PSS, Mainz, Germany) with molecular weights from $342 \mathrm{Da}$ to $805000 \mathrm{Da}$ was used as standard. Molecular weight was determined using a refractive index increment dn/dc of 0.072 (Ciric et al., 2014), and a Debye plot. 


\section{Results \& Discussion}

\subsection{GTFB- $\triangle N$ activity and synthesis of IMMPS}

The hydrolytic activity of the GTFB- $\triangle \mathrm{N}$ enzyme was tested with a GOPOD based activity assay and is comparable to the reported activity in a previous study (Bai et al., 2015a). IMMPs were produced from (waxy) potato starch, (waxy) maize starch, (waxy) rice starch, sweet potato starch and wheat starch using GTFB- $\Delta \mathrm{N}$ with an average reaction yield of $84 \%(\mathrm{w} / \mathrm{w})$. Specific yield per sample can be found in the supplementary information (7.1).

To investigate the number of $\alpha-(1 \rightarrow 4)$ and $\alpha-(1 \rightarrow 6)$ linkages ${ }^{1} \mathrm{H}$ NMR was used. Typical ${ }^{1} \mathrm{H}$ NMR spectra for IMMPs in $\mathrm{D}_{2} \mathrm{O}$ at $340 \mathrm{~K}$ are shown in Figure 1 , typical ${ }^{1} \mathrm{H}$ NMR spectra of maltodextrins before and after GTFB- $\Delta \mathrm{N}$ treatment were published previously by Bai et al. (2015a) and Leemhuis et al. (2014). The most notable peaks are at $5.4 \mathrm{ppm}$ and $5.0 \mathrm{ppm}$ corresponding to respectively $\alpha-(1 \rightarrow 4)$ and $\alpha-(1 \rightarrow 6)$ linkages (Dobruchowska et al., 2012; Leemhuis et al., 2014; van Leeuwen et al., 2008). The lack of measurable signals at \pm 5.2 and $\pm 4.6 \mathrm{ppm}$ corresponding to the $\alpha$ - and $\beta$-reducing ends respectively, indicate that the produced IMMPs are large molecules. The total $\alpha-(1 \rightarrow 6)$ content measured with ${ }^{1} \mathrm{H}$ NMR includes both $\alpha-(1 \rightarrow 4,6)$ branching points and linear $\alpha-(1 \rightarrow 6)$ linkages. The obtained total $\alpha-(1 \rightarrow 6)$ content percentages are compiled in Table 1.

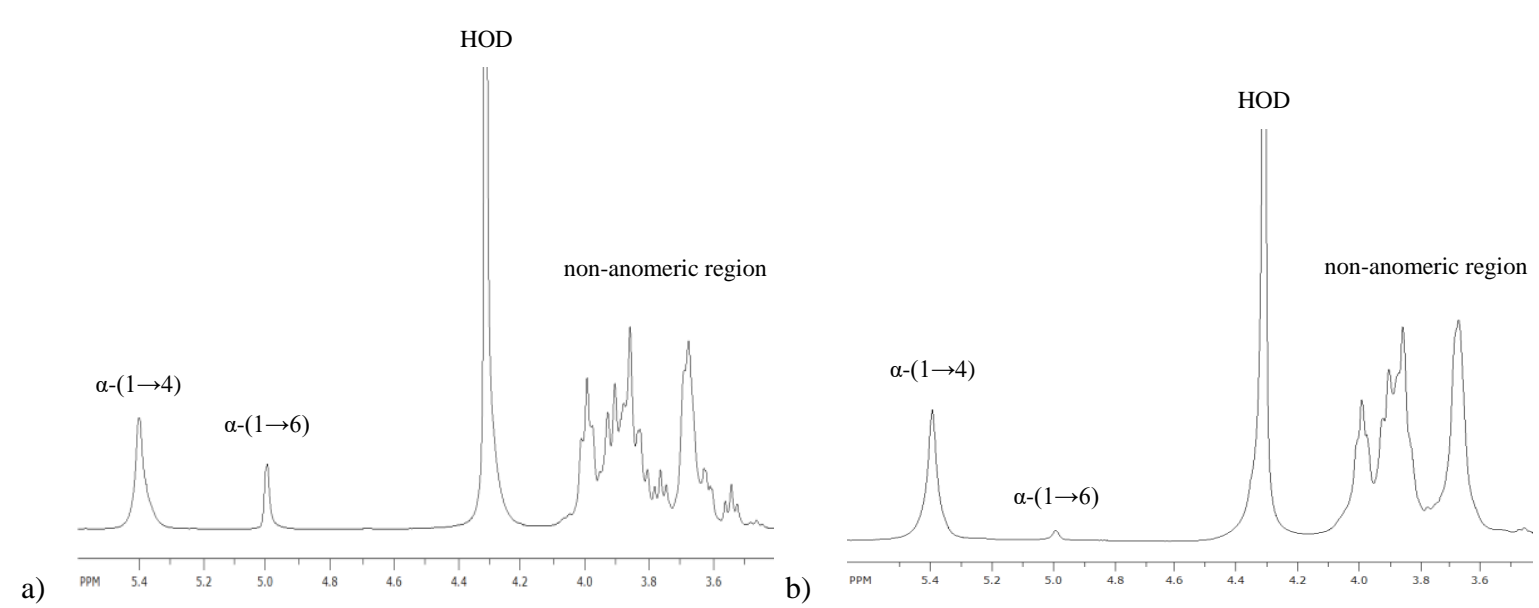

Figure 1. $500 \mathrm{MHz}{ }^{1} \mathrm{H}$ NMR spectra $\left(\mathrm{D}_{2} \mathrm{O}, 340 \mathrm{~K}\right)$ of GTFB- $\Delta \mathrm{N}$ modified starches; (a) potato IMMP and (b) waxy potato IMMP, with a total $\alpha-(1 \rightarrow 6)$ content of $25 \%$ and $5 \%$ respectively.

Literature values for the amylose content, degree of branching and the average amylopectin chain length of the starch substrates are depicted in Table 1. Since the amount GTFB- $\Delta$ N hydrolytic activity can also be used as an indicator for overall GTFB 
efficiency (Bai et al., 2015a), in addition to the total $\alpha-(1 \rightarrow 6)$ content, also the amount of free glucose was used to determine the extent of GTFB- $\Delta \mathrm{N}$ modification. The extent of GTFB- $\triangle \mathrm{N}$ modification determined with ${ }^{1} \mathrm{H}$ NMR was compared to previous results (Table 1).

Table 1. Measured values of free glucose (GOPOD) and $\alpha-(1 \rightarrow 6)$ content $\left({ }^{1} \mathrm{H}\right.$ NMR) of produced IMMPs compared to the substrate starch's amylose content, amylopectin chain length. ${ }^{j}$ (Jane et al., 1999) ${ }^{l}$ (Leemhuis et al., 2014) ${ }^{c}\left(\right.$ Chen et al., 2003) ${ }^{h}$ (Hizukuri et al., 1983)

\begin{tabular}{|c|c|c|c|c|c|c|}
\hline \multirow{3}{*}{$\begin{array}{l}\text { Starch type } \\
\text { Potato }\end{array}$} & \multicolumn{3}{|c|}{ Substrate } & \multicolumn{3}{|c|}{ IMMP } \\
\hline & \multirow{2}{*}{$\begin{array}{c}\begin{array}{c}\text { Amylose } \\
\text { content } \\
\text { (apparent) }\end{array} \\
36^{j}\end{array}$} & \multirow{2}{*}{$\begin{array}{c}\begin{array}{c}\text { Degree of } \\
\text { branching } \\
(\%)\end{array} \\
3.1^{l}\end{array}$} & \multirow{2}{*}{$\begin{array}{c}\text { Average } \\
\text { amylopectin } \\
\text { chain length }\end{array}$} & \multirow{2}{*}{$\begin{array}{c}\begin{array}{c}\text { Free } \\
\text { glucose } \\
(\%)\end{array} \\
1.6\end{array}$} & \multicolumn{2}{|c|}{$\begin{array}{c}{ }^{1} \mathrm{H} \text { NMR } \\
\text { total } \alpha-(1 \rightarrow 6) \\
\text { content }(\%)\end{array}$} \\
\hline & & & & & 25 & $28^{l}$ \\
\hline Waxy potato & 0 & $4.0^{l}$ & - & 0.8 & 5 & $14^{l}$ \\
\hline Maize & $29^{j}$ & $3.6^{l}$ & $24^{j}$ & 1 & 19 & $21^{l}$ \\
\hline Waxy maize & $0^{j}$ & $4.8^{l}$ & $24^{j}$ & 0.6 & 6 & $7^{l}$ \\
\hline Rice & $25^{j}$ & $4.1^{l}$ & $23^{j}$ & 0.8 & 13 & $13^{l}$ \\
\hline Waxy rice & $0^{j}$ & $4.9^{l}$ & $19^{j}$ & 0.5 & 6 & $7^{l}$ \\
\hline Sweet potato & $19^{c}$ & - & $21^{h}$ & 1.2 & 18 & - \\
\hline Wheat & $29^{j}$ & $3.7^{l}$ & $23^{j}$ & 1.5 & 23 & $22^{l}$ \\
\hline
\end{tabular}

When a comparison is made between amylose-rich (normal) and amylose-free (waxy) starches it becomes clear that a higher amylose content in the substrate correlates to a higher amount of total $\alpha-(1 \rightarrow 6)$ content and free glucose in the corresponding IMMP reaction mixture. Free glucose measured after modification showed an average of $0.6 \%$ for IMMPs derived from amylose-free starches, while an average of $1.2 \%$ was found for IMMPs derived from amylose-rich starches. The total $\alpha-(1 \rightarrow 6)$ content of rice IMMP is somewhat lower compared to IMMPs derived from starches with a similar amylose content. This is probably related to the higher degree of branching and the branched nature of rice amylose (Takeda et al., 1993). The extent of modification is consistent with previous literature, with the exception of the high total $\alpha-(1 \rightarrow 6)$ content for waxy potato IMMP reported by Leemhuis et al. (2014). Apart from this outlier, total $\alpha-(1 \rightarrow 6)$ content per starch type is in line with earlier reported results, which indicates that amylose content in the substrate determines the quantity of $\alpha-(1 \rightarrow 6)$ linkages produced by the GTFB- $\Delta N$ enzyme. The low total $\alpha-(1 \rightarrow 6)$ content in IMMPs derived from amylose-free starches indicates that amylose-free starches are hardly affected by GTFB$\Delta \mathrm{N}$ transferase activity. 


\subsection{Influence of GTFB- $\triangle N$ modification on product size and solubility}

The experimental results indicate that especially amylose is involved in the formation of $\alpha-(1 \rightarrow 6)$ linkages, which is in accordance with corresponding values found in literature (Table 1). However, information about the effect of amylose on the molecular weight distribution of IMMPs is still lacking. Since different starches vary in amylose content, it is important to study what effect this has on the final molecular weight distribution of the resulting IMMP. Therefore the size distribution of starches before and after GTFB- $\Delta$ N modification was investigated with GPC (DMSO elution).

As representative example of GPC-MALLS analysis, the elution patterns of gelatinized sweet potato starch and sweet potato IMMP are depicted in Figure 2. The concentration $(\mathrm{g} / \mathrm{L})$ of sweet potato starch and sweet potato IMMP is plotted against the elution volume and can be read out on the right axis. An indication of the molecular weight on a specific elution volume can be read out in either the MALLS signal or the pullulan standard plotted on the left axis.

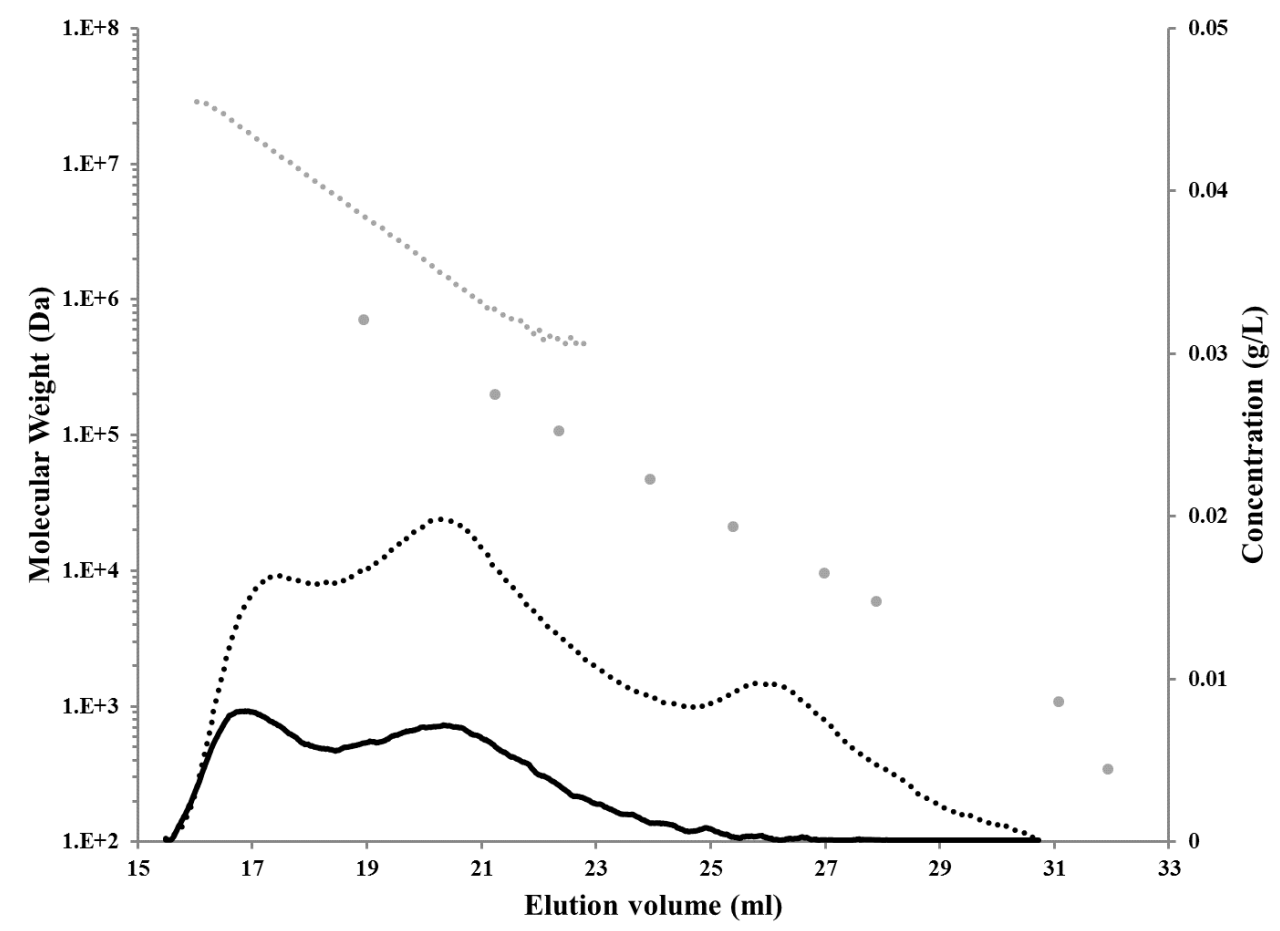

Figure 2. GPC-MALLS (DMSO elution) of sweet potato starch and sweet potato IMMP. The concentration, derived from the RI signal, of sweet potato starch ( _ $)$ and sweet potato IMMP (....) is plotted on the right axis. The molecular weight of the pullulan standard $(\bullet)$ and the calculated molecular weight (Da) of sweet potato $\operatorname{IMMP}(\ldots)$ is plotted on the left axis.

When comparing the elution pattern of the gelatinized starches to the elution pattern of their respective IMMPs, it can be noted that the surface area of the starch substrate is 
much smaller than the surface area of its respective IMMP (Figure 2 and supplementary information 7.2). Since an equal amount of both samples was injected on the column, the difference in surface area indicates that gelatinized starch is not eluting completely. Gelatinized starch and especially the amylopectin fraction are known to display low mass recoveries in most chromatographic methods (Bello-Pérez et al., 1998). Mostly due to interaction of the large amylopectin fraction, reported $\mathrm{M}_{\mathrm{w}}$ values in the range of $2-700 \times 10^{6} \mathrm{Da}$ (Pérez \& Bertoft, 2010), with the column material. The incomplete elution of the starch substrate therefore results in an underestimation of its respective amylopectin fraction (Bello-Pérez et al., 1998).

IMMPs made from amylose-rich starches, such as sweet potato IMMP shown in Figure 2 , show an additional peak starting at $25 \mathrm{~mL}$ elution volume ( $<50 \mathrm{kDa}$ ). IMMPs from amylose-free starches do not show an elevated population at $25 \mathrm{~mL}$ elution volume (supplementary information 7.2). Since amylose-rich starches produce IMMPs with relatively high $\alpha-(1 \rightarrow 6)$ contents, the more pronounced lower molecular weight fraction at $25 \mathrm{~mL}$ elution volume might contain most of newly introduced $\alpha-(1 \rightarrow 6)$ linkages by the GTFB- $\Delta$ N enzyme. Despite the low $\alpha-(1 \rightarrow 6)$ content of IMMPs made from amylose-free starches (Table 1), GTFB- $\Delta$ N modification of these amylose-free starches still results in a noticeable increase in solubility and elutability (supplementary information 7.2). Apparently, this mostly hydrolytic modification with GTFB- $\Delta \mathrm{N}$ is sufficient to make starch amylopectin fully elutable on GPC (DMSO-elution). Although IMMPs of amylose-rich and amylose-free starches elute equally well, it can be concluded that the presence of amylose in results in the formation of a more pronounced lower molecular weight fraction in the respective IMMP.

\subsection{Preparative fractionation of IMMPs}

In order to determine the composition of the different molecular weight populations, IMMPs were fractionated on a preparative scale using size exclusion chromatography. The preparative fractionation was performed in an aqueous setting in order to obtain an accurate mass balance of the collected fractions after concentration and lyophilisation (supplementary information 7.3). The IMMPs and their respective HMW, MMW and LMW fractions were further studied on the GPC-MALLS system with DMSO elution. As a representative result of IMMP fractionation, the fractionation of sweet potato IMMP is depicted in Figure 3. In order to get a realistic impression on the different molecular weight populations, response per fraction was adjusted for the corresponding fraction's yield. 


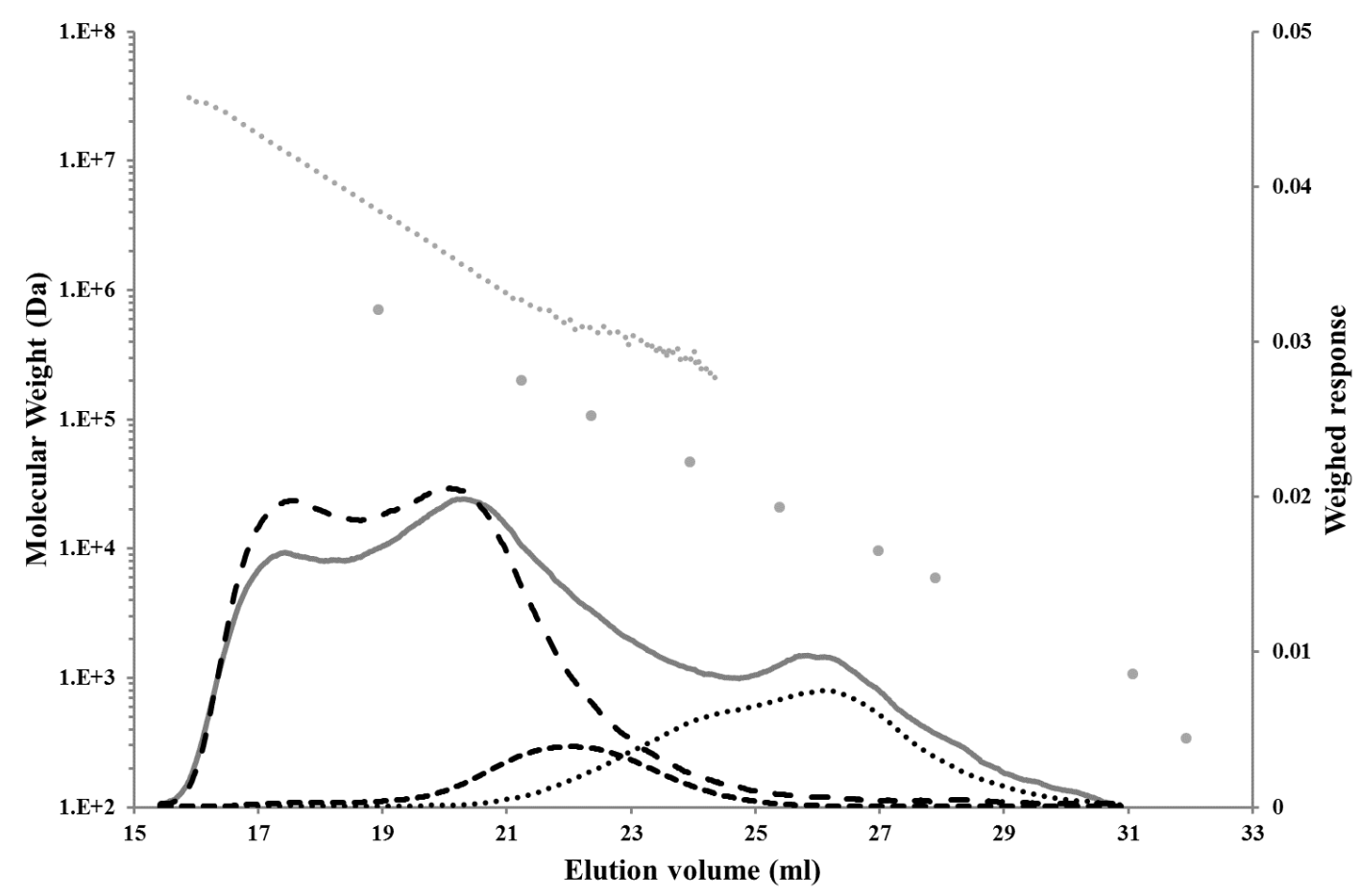

Figure 3. GPC-MALLS (DMSO elution) of sweet potato IMMP ( ) and its respective high $(\boldsymbol{-}-)$, medium (_- $)$ and low (.....) molecular weight fractions obtained after preparative fractionation. Molecular weight (Da) of the pullulan standard ( • ) and the unfractionated sweet potato IMMP (... ) is plotted on the left axis. The HMW, MMW and LMW responses were weighed according to the obtained fractionation yields and plotted on the right axis.

Fractionation yield and GPC-MALLS responses for the other IMMPs (supplementary information $7.3 \&$ \% 7 ) follow similar trends. IMMPs produced from amylose-rich starches contain a larger LMW fraction as above described for the elution of the unfractionated IMMPs. Since the MMW-fraction is a small intermediate fraction containing both HMW and LMW compounds, it is not taken into account in the further analysis. The original $\alpha-(1 \rightarrow 6)$ content of the respective IMMP can be recalculated, when combining the fractionation yields of the HMW, MMW and LMW fraction with their corresponding total $\alpha-(1 \rightarrow 6)$ content (supplementary information 7.3). Since the recalculated $\alpha-(1 \rightarrow 6)$ content closely resembles the original $\alpha-(1 \rightarrow 6)$ content, it means that losses in fractionation yield occurred over all fractions equally, indicating that the fractionation was successful.

The total $\alpha-(1 \rightarrow 6)$ content of unfractionated IMMPs compared to their respective HMW and LMW fractions is depicted in Figure 4. It can be concluded that the total $\alpha$ $(1 \rightarrow 6)$ content of an IMMP fraction is dependent on its molecular weight, especially for IMMPs derived from amylose-rich starches. IMMPs derived from amylose-free starches show little variation in $\alpha-(1 \rightarrow 6)$ content in their respective HMW and LMW fractions, while the LMW-fractions of IMMPs derived from amylose-rich starches 
contain a high total $\alpha-(1 \rightarrow 6)$ content. This means that the total $\alpha-(1 \rightarrow 6)$ content of an unfractionated IMMP derived from an amylose-rich starch is disproportionally influenced by its LMW-fraction (Figure 4).

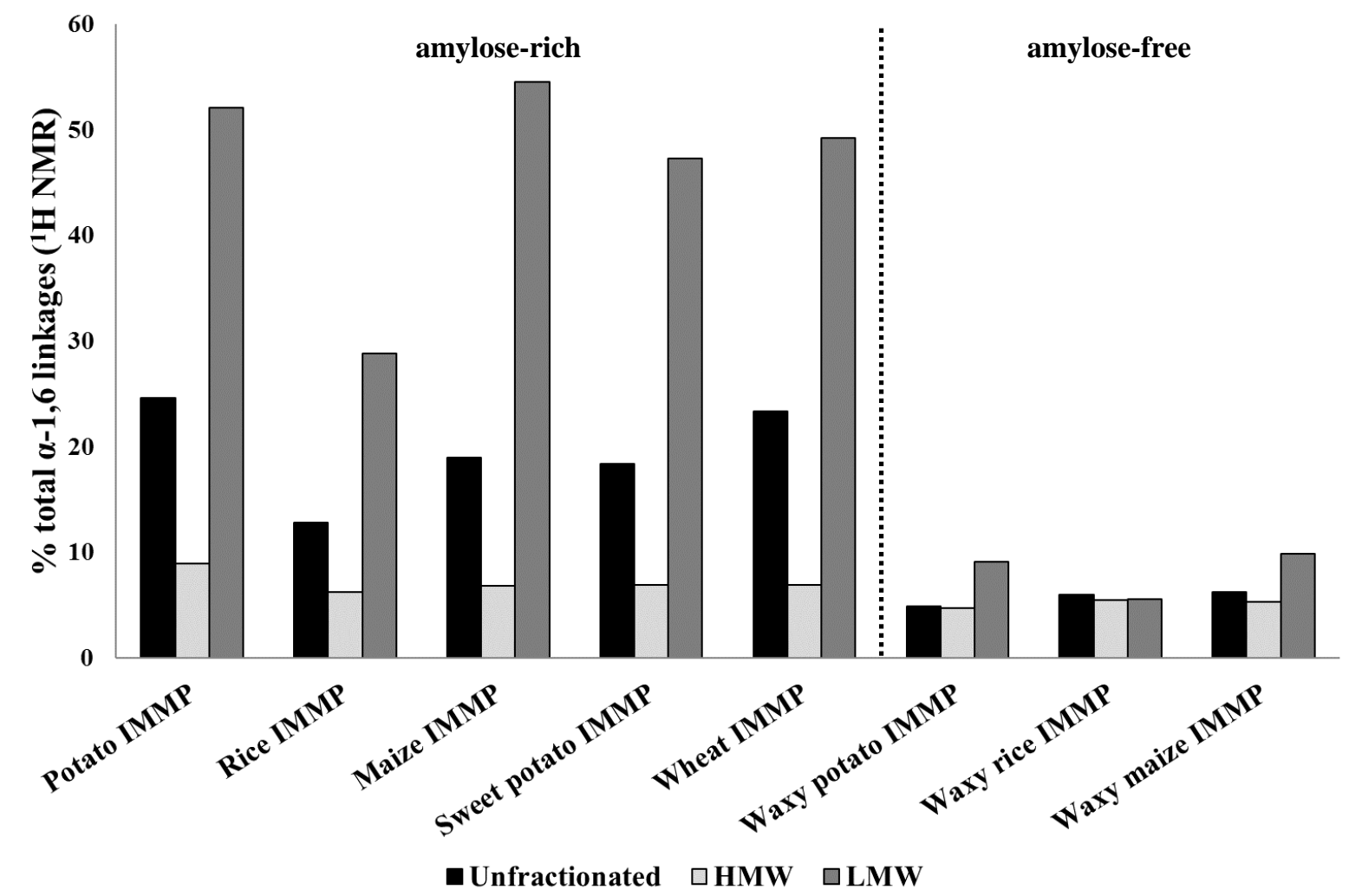

Figure 4. Total $\alpha-(1 \rightarrow 6)$ content ( ${ }^{1} \mathrm{H}$ NMR) of unfractionated IMMPs versus their respective IMMP HMW and LMW fractions.

When observing the IMMP LMW fractions derived from amylose-rich starches in Figure 4, there seems to be a limitation to the extent of modification. The IMMP LMW fractions derived from amylose-rich starches seem to be limited to a total $\alpha-(1 \rightarrow 6)$ content of about 50\%. Since virtually linear substrates such as amylose $\mathrm{V}$ and fully debranched starch are known to be capable of reaching total $\alpha-(1 \rightarrow 6)$ contents of $91 \%$ and $96 \%$ respectively (Leemhuis et al, 2014). It seems that the IMMP LMW fractions still contain a limiting factor that prevents complete modification.

\subsection{Linkage analysis on IMMPs and their respective fractions}

While ${ }^{1} \mathrm{H}$ NMR has been useful in determining the total $\alpha-(1 \rightarrow 6)$ content of IMMPs, it is not suitable to distinguish branching $\alpha-(1 \rightarrow 4,6)$ linkages from linear $\alpha-(1 \rightarrow 6)$ linkages in a quantifiable manner. The difference between these linkage types is important for the characterization of IMMPs, since the GTFB- $\Delta \mathrm{N}$ enzyme introduces linear $\alpha-(1 \rightarrow 6)$ linkages and starch only contains $\alpha-(1 \rightarrow 4)$ and $\alpha-(1 \rightarrow 4,6)$ linkages. 
Therefore, permethylation analysis was used to determine the distribution of native $\alpha$ $(1 \rightarrow 4,6)$ and newly introduced $\alpha-(1 \rightarrow 6)$ linkages in the collected IMMP fractions. Results of the linkage composition analysis of IMMP HMW and LMW fractions are depicted in Figure 5, additional information about the smaller IMMP MMW fraction can be found in supplementary information (7.5).

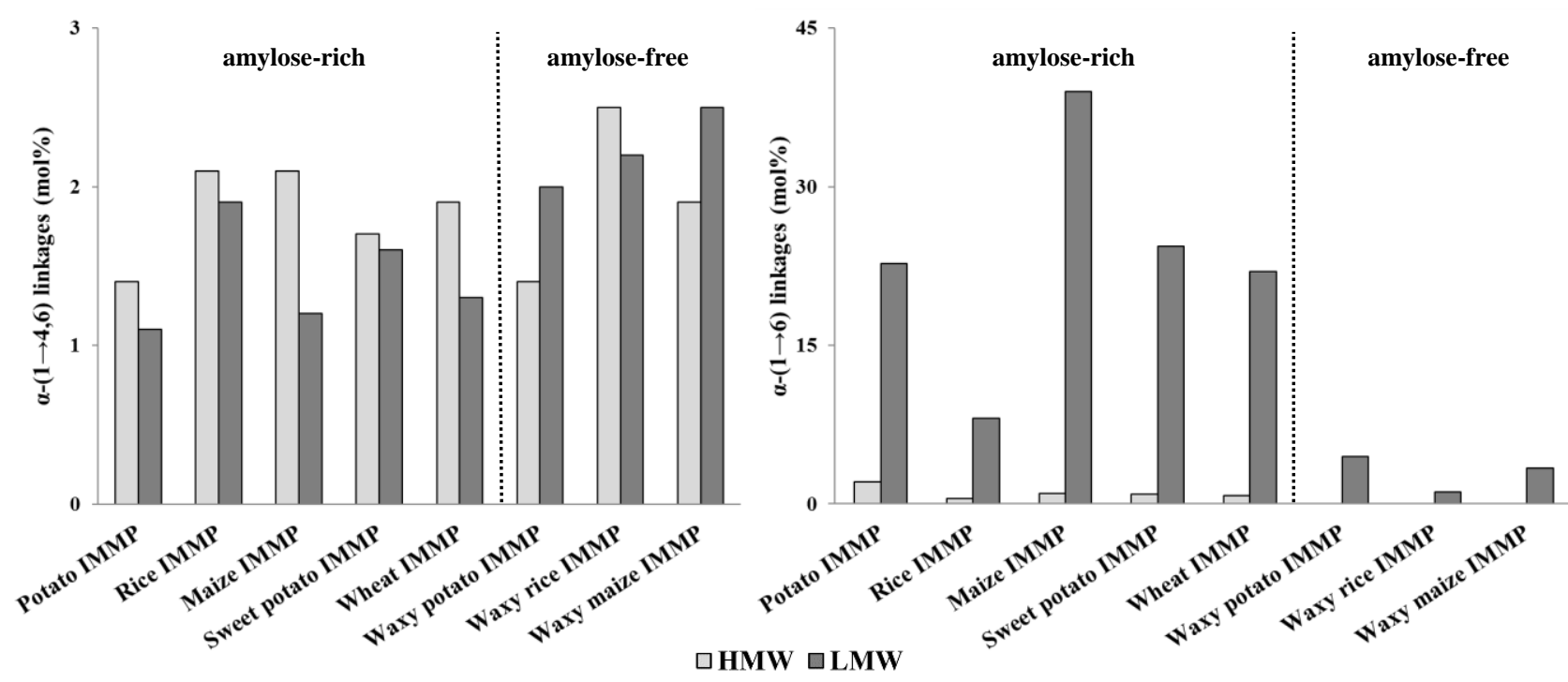

Figure 5. The relative amount of branched $\alpha-(1 \rightarrow 4,6)$ - and linear $\alpha-(1 \rightarrow 6)$-linkages in the IMMP HMW and LMW fractions determined by permethylation analysis.

Most of the linear $\alpha-(1 \rightarrow 6)$ linkages introduced by the GTFB- $\Delta$ N enzyme end up in the IMMP LMW fraction (Figure 5). When we relate this outcome to the structure of the GTFB enzyme (Bai et al., 2016a), the LMW fraction is likely to suffer less steric hindrance compared the HMW fraction, and is therefore able to diffuse faster into the GTFB- $\Delta \mathrm{N}$ acceptor sub-site. The effect of the faster diffusion rate is, in turn, amplified by the fact that the LMW material becomes a better acceptor after the first $\alpha-(1 \rightarrow 6)$ linkage is introduced, since the reported GTFB- $\Delta \mathrm{N}$ transglycosylation factor is higher for $\alpha-(1 \rightarrow 6)$ linked acceptors compared to $\alpha-(1 \rightarrow 4)$ linked acceptors (Bai et al., 2015a; Leemhuis et al., 2014). The combined effect of faster diffusion of LMW material and transglycosylation preference for $\alpha-(1 \rightarrow 6)$ linkages explains why the LMW fraction functions as a better acceptor for GTFB- $\Delta \mathrm{N}$ transferase activity.

The amount of $\alpha-(1 \rightarrow 4,6)$ branching points is equally divided over IMMP HMW and LMW fractions (Figure 6). The presence of branching points in the LMW fraction reveals why the GTFB- $\Delta$ N enzyme is not able to fully convert the LMW fraction into linear $\alpha-(1 \rightarrow 6)$ linkages (Figure 4$)$, since the mostly exo-acting nature of the GTFB- $\Delta \mathrm{N}$ enzyme (Bai et al., 2016a), results in a negative correlation between the amount of branching points and the total $\alpha-(1 \rightarrow 6)$ content (Leemhuis et al., 2014). The amount of 
branching points present in the IMMP LMW fraction also indicates that we cannot completely rule out GTFB- $\Delta \mathrm{N}$ hydrolytic endo-activity on the inner chains of amylopectin, as shown previously for linear oligosaccharide substrates (Bai et al., 2016a).

IMMP HMW fractions derived from amylose-free starches do not contain any linear $\alpha$ $(1 \rightarrow 6)$ linkages (Figure 6). Since the size and elutability of amylose-free starches are affected by the GTFB- $\Delta \mathrm{N}$ modification (supplementary information 7.2), the absence of linear $\alpha-(1 \rightarrow 6)$ linkages shows that GTFB- $\Delta \mathrm{N}$ only affects the amylopectin fraction in amylose-free starches by partial hydrolysis. Previous studies show that $\alpha-(1 \rightarrow 4,6)$ branching points in starch substrates have a limiting effect on GTFB- $\Delta$ N activity (Bai et al., 2016a; Leemhuis et al., 2014). This study proves that the GTFB- $\Delta$ N enzyme only displays hydrolytic activity on HMW amylopectin fractions in the absence of amylose. However, the partial hydrolysis of the HMW fraction of amylose-free starches does lead to the introduction of a limited amount of linear $\alpha-(1 \rightarrow 6)$ linkages into hydrolytic debris of amylopectin in the LMW fraction.

The presence of newly introduced $\alpha-(1 \rightarrow 6)$ linkages in the IMMP HMW fractions derived from amylose-rich starches (Figure 6) shows that, in the presence of amylose, GTFB- $\Delta \mathrm{N}$ is able to incorporate linear $\alpha-(1 \rightarrow 6)$ linkages into the former amylopectin fraction. The relatively high amount of linear $\alpha-(1 \rightarrow 6)$ linkages in the potato IMMP HMW fraction compared to the other amylose-rich starches (Figure 6), indicates that potato amylopectin is a better acceptor for the GTFB- $\Delta$ N deposition of linear $\alpha-(1 \rightarrow 6)$ linkages. This is possibly related to the high average amylopectin chain length of potato amylopectin (Table 1). All in all, it can be concluded that the presence of amylose, or a similar linear $\alpha-(1 \rightarrow 4)$ linked substrate, is a prerequisite for the incorporation of linear $\alpha-(1 \rightarrow 6)$ linkages into the branched IMMP HMW fraction and that the amount of incorporated $\alpha-(1 \rightarrow 6)$ linkages seems to be positively related to average amylopectin chain length.

\subsection{IMMP structure and possible physicochemical properties}

We showed that the GTFB- $\Delta$ N modification of an amylose-rich starch leads to a high amount of linear $\alpha-(1 \rightarrow 6)$ linkages in the IMMP LMW fraction and a small amount of linear $\alpha-(1 \rightarrow 6)$ linkages being incorporated into the branched amylopectin molecule (Figure 6, supplementary information 7.5). Assuming that hydrolysis is taking place alongside GTFB- $\Delta \mathrm{N}$ transferase activity (Bai et al., 2015a), as can also be concluded from the reduction in molecular weight after GTFB- $\Delta \mathrm{N}$ modification (Figure 2, 
supplementary information 7.2). We propose the following structure for an amyloserich starch modified with the GTFB- $\Delta \mathrm{N}$ enzyme (Figure 6).

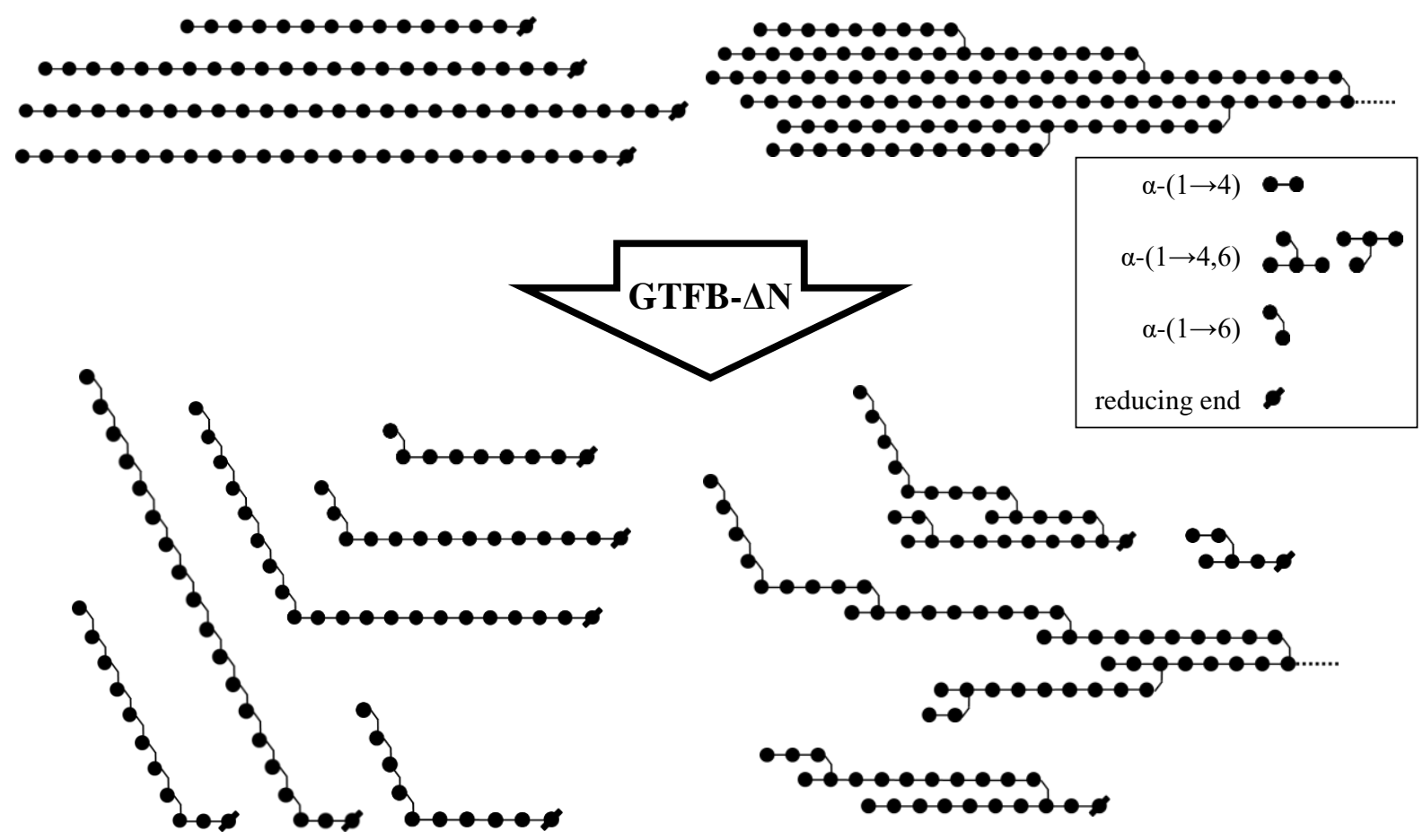

Figure 6. Schematical overview of an IMMP derived from an amylose-rich starch.

All in all, IMMPs are found to be more soluble and elutable than their respective starch substrates (Figure 2, supplementary information 7.2). The hydrolysis of linear $\alpha-(1 \rightarrow 4)$ linked glucan chains severely alters the physicochemical properties of the starch substrate, since a minimum $\alpha-(1 \rightarrow 4)$ chain length of DP 10 is required for the double helices responsible for starch's traditional gelling and retrogradation properties (Gidley \& Bulpin, 1989; Pérez \& Bertoft, 2010; Pfannemüller, 1987). The lack of rigidity in the newly introduced $\alpha-(1 \rightarrow 6)$ linked chains results, in turn, in more solute-solvent and less solute-solute hydrogen bonding, compared an $\alpha-(1 \rightarrow 4)$ linked compound (Best et al., 2001). The lack of internal hydrogen bonding in the $\alpha-(1 \rightarrow 6)$ linked chains prevents the formation of less soluble structures that are common in glucans, such as the $\alpha-(1 \rightarrow 4)$ helix or the $\beta-(1 \rightarrow 4)$ fibril in starch and cellulose respectively (Pérez \& Bertoft, 2010; Nishiyama, 2009). The lack of internal hydrogen bonding however, does introduce other possibilities for higher order structuring, such as the formation of crystal polymorphs similar to the types that are observed in dextran (Guizard et al., 1985a, 1985b). 


\section{Conclusions}

In this study we showed that $\alpha-(1 \rightarrow 4,6)$ linkages are homogeneously distributed across IMMP molecular weight fractions, while linear $\alpha-(1 \rightarrow 6)$ linkages introduced by the GTFB- $\Delta \mathrm{N}$ enzyme are distributed in a heterogeneous matter. Starch amylose is responsible for creating a more pronounced IMMP LMW fraction rich in linear $\alpha-(1 \rightarrow 6)$ linkages. While $\alpha-(1 \rightarrow 4,6)$ branching points, present in both amylopectin and amylose, have a limiting effect on $\alpha-(1 \rightarrow 6)$ linkage formation. Additionally, by studying amylose-rich and amylose-free substrates, we learned that the presence of amylose during GTFB- $\Delta N$ modification determines whether linear $\alpha-(1 \rightarrow 6)$ linkages are introduced in the amylopectin molecule. All in all, this study shows that an investigation of the complete IMMP molecular weight range and its respective linkage composition is essential for creating a deeper understanding of IMMP formation and its relation to the respective starch origins. Although some facets of IMMP substructure, such as the average chain length of the introduced $\alpha-(1 \rightarrow 6)$ linkages, are still to be investigated. This study of the substrate-product relation has provided a foothold for the directed synthesis of IMMPs from starch substrates.

\section{Acknowledgements}

This project (TKI-2013-B) is jointly funded by AVEBE and TKI as coordinated by the Carbohydrate Competence Center (CCC-ABC; www.cccresearch.nl). The authors thank Pieter de Waard (Wageningen NMR centre), Albert Woortman and Katja Loos (University of Groningen) for help with the analysis and Melliana Jonathan (Wageningen University \& Research) for the useful discussions. 


\section{References}

Ayudhaya, P., Pongsawasdi, P., Laohasongkram, K., \& Chaiwanichsiri, S. (2016). Properties of Cassava Starch Modified by Amylomaltase from Corynebacterium glutamicum. Journal of Food Science, 81(6), 1363-1369. https://doi.org/10.1111/1750-3841.13305

Bai, Y., Böger, M., van der Kaaij, R. M., Woortman, A. J. J., Pijning, T., van Leeuwen, S. S., van Bueren A. L., \& Dijkhuizen, L. (2016b). Lactobacillus reuteri Strains Convert Starch and Maltodextrins into Homoexopolysaccharides Using an Extracellular and Cell-Associated 4,6- $\alpha-$ Glucanotransferase. Journal of Agricultural and Food Chemistry, 64(14), 2941-2952. https://doi.org/10.1021/acs.jafc.6b00714

Bai, Y., Gangoiti, J., Dijkstra, B. W., Dijkhuizen, L., \& Pijning, T. (2016a). Crystal Structure of 4,6- $\alpha-$ Glucanotransferase Supports Diet-Driven Evolution of GH70 Enzymes from $\alpha$-Amylases in Oral Bacteria. Structure, 1-12. https://doi.org/10.1016/j.str.2016.11.023

Bai, Y., van der Kaaij, R. M., Leemhuis, H., Pijning, T., Leeuwen, S. S. van, Jin, Z., \& Dijkhuizen, L. (2015a). Biochemical characterization of the Lactobacillus reuteri glycoside hydrolase family 70 GTFB type of 4,6- $\alpha$-glucanotransferase enzymes that synthesize soluble dietary starch fibers. Applied and Environmental Microbiology, 81(20), 7223-7232. https://doi.org/10.1128/AEM.01860-15

Bai, Y., van der Kaaij, R. M., Woortman, A. J. J., Jin, Z., \& Dijkhuizen, L. (2015b). Characterization of the 4,6- $\alpha$-glucanotransferase GTFB enzyme of Lactobacillus reuteri 121 isolated from inclusion bodies. BMC Biotechnology, 15, 49. https://doi.org/10.1186/s12896-015-0163-7

Bello-Pérez, L. A., Roger, P., Baud, B., \& Colonna, P. (1998). Macromolecular Features of Starches Determined by Aqueous High-performance Size Exclusion Chromatography. Journal of Cereal Science, 27. https://doi.org/10.1006/jcrs.1998.0186

Best, R. B., Jackson, G. E., \& Naidoo, K. J. (2001). Molecular Dynamics and NMR Study of the $\alpha$ $(1 \rightarrow 4)$ and $\alpha-(1 \rightarrow 6)$ Glycosidic Linkages: Maltose and Isomaltose. The Journal of Physical Chemistry B, 105, 4742-4751. https://doi.org/10.1021/jp0040704

Bissaro, B., Monsan, P., Fauré, R., \& O’Donohue, M. J. (2015). Glycosynthesis in a waterworld: new insight into the molecular basis of transglycosylation in retaining glycoside hydrolases.

Biochemical Journal, 467(1), 17-35. https://doi.org/10.1042/BJ20141412

Chen, Z., Schols, H. ., \& Voragen, A. G. . (2003). Physicochemical Properties of Starches Obtained from Three Varieties of Chinese Sweet Potatoes. Food and Chemical Toxicology, 68(2), 431437. https://doi.org/10.1111/j.1365-2621.2003.tb05690.x

Chockchaisawasdee, S., \& Poosaran, N. (2013). Production of isomaltooligosaccharides from banana flour. Journal of the Science of Food and Agriculture, 93(1), 180-186. https://doi.org/10.1002/jsfa.5747

Ciric, J., Woortman, A. J. J., \& Loos, K. (2014). Analysis of isoamylase debranched starches with size exclusion chromatography utilizing PFG columns. Carbohydrate Polymers, 112, 458-461. https://doi.org/10.1016/j.carbpol.2014.05.093

Crini, G. (2014). Review: A history of cyclodextrins. Chemical Reviews, 114(21), 10940-10975. https://doi.org/10.1021/cr500081p

Dijkhuizen, L., Van, D. M. M. J. E. C., Kamerling, J. P., Leemhuis, R. J., Kralj, S., \& Dobruchowska, J. M. (2010). Gluco-oligosaccharides comprising $\alpha-(1 \rightarrow 4)$ and $\alpha-(1 \rightarrow 6)$ glycosidic bonds, use thereof, and methods for providing them. WO2010128859A2. 
Dobruchowska, J. M., Gerwig, G. J., Kralj, S., Grijpstra, P., Leemhuis, H., Dijkhuizen, L., \& Kamerling, J. P. (2012). Structural characterization of linear isomalto-/malto-oligomer products synthesized by the novel GTFB 4,6- $\alpha$-glucanotransferase enzyme from Lactobacillus reuteri 121. Glycobiology, 22(4), 517-528. https://doi.org/10.1093/glycob/cwr167

Funane, K., Ichinose, H., Araki, M., Suzuki, R., Kimura, K., Fujimoto, Z., Kobayashi, M., \& Kimura, A. (2014). Evidence for cycloisomaltooligosaccharide production from starch by Bacillus circulans T-3040. Applied Microbiology and Biotechnology, 98(9), 3947-3954. https://doi.org/10.1007/s00253-014-5515-z

Gidley, M. J., \& Bulpin, P. V. (1989). Aggregation of Amylose in Aqueous Systems: The Effect of Chain Length on Phase Behavior and Aggregation Kinetics. Macromolecules, 22(13), 341-346. https://doi.org/10.1021/ma00191a062

Goffin, D., Delzenne, N., Blecker, C., Hanon, E., Deroanne, C., \& Paquot, M. (2011). Will isomaltooligosaccharides, a well-established functional food in Asia, break through the European and American market? The status of knowledge on these prebiotics. Critical Reviews in Food Science and Nutrition, 51(5), 394-409. https://doi.org/10.1080/10408391003628955

Grimaud, F., Lancelon-Pin, C., Rolland-Sabaté, A., Roussel, X., Laguerre, S., Viksø-Nielsen, A., Putaux, J. L., Guilois, S., Buléon, A., D'Hulst, C., \& Potocki-Véronèse, G. (2013). In vitro synthesis of hyperbranched $\alpha$-glucans using a biomimetic enzymatic toolbox. Biomacromolecules, 14(2), 438-447. https://doi.org/10.1021/bm301676c

Guizard, C., Chanzy, H., \& Sarko, A. (1985a). The molecular and crystal structure of dextrans: A combined electron and X-ray diffraction study. Journal of Molecular Biology, 183(8), 397-408. https://doi.org/10.1016/0022-2836(85)90010-5

Guizard, C., Chanzy, H., \& Sarko, A. (1985b). The molecular and crystal structure of dextrans: A combined electron and X-ray diffraction study. II. A low temperature, hydrated polymorph. Journal of Molecular Biology, 183(3), 397-408. https://doi.org/10.1016/0022-2836(85)90010-5

Guzmán-Maldonado, H., \& Paredes-López, O. (1995). Amylolytic enzymes and products derived from starch: a review. Critical Reviews in Food Science and Nutrition, 35(5), 373-403. https://doi.org/10.1080/10408399509527706

Hizukuri, S., Kaneko, T., \& Takeda, Y. (1983). Measurement of the chain length of amylopectin and its relevance to the origin of crystalline polymorphism of starch granules. BBA - General Subjects, 760(1), 188-191. https://doi.org/10.1016/0304-4165(83)90142-3

Hu, Y., Ketabi, A., Buchko, A., \& Gänzle, M. G. (2013). Metabolism of isomalto-oligosaccharides by Lactobacillus reuteri and bifidobacteria. Letters in Applied Microbiology, 57(2), 108-114. https://doi.org/10.1111/lam.12076

Jane, J. (1999). Effects of amylopectin branch chain-length and amylose content on the gelatinization and pasting properties of starch. Cereal Chemistry, 52(3258), 555. https://doi.org/10.1094/CCHEM.1999.76.5.629

Kadokawa, J. I. (2011). Precision Polysaccharide Synthesis Catalyzed by Enzymes. Chemical Reviews, 111(7), 4308-4345. https://doi.org/10.1021/cr100285v

Kajiura, H., Takata, H., Kuriki, T., \& Kitamura, S. (2010). Structure and solution properties of enzymatically synthesized glycogen. Carbohydrate Research, 345(6), 817-824. https://doi.org/10.1016/j.carres.2010.01.013

Kaulpiboon, J., Rudeekulthamrong, P., Watanasatitarpa, S., Ito, K., \& Pongsawasdi, P. (2015). Synthesis of long-chain isomaltooligosaccharides from tapioca starch and an in vitro investigation of their prebiotic properties. Journal of Molecular Catalysis B: Enzymatic, 120, 
127-135. https://doi.org/10.1016/j.molcatb.2015.07.004

Kralj, S., Grijpstra, P., van Leeuwen, S. S., Leemhuis, H., Dobruchowska, J. M., van der Kaaij, R. M., Malik, A., Oetari, A., Kamerling, J. P., \& Dijkhuizen, L. (2011). 4,6- $\alpha$-glucanotransferase, a novel enzyme that structurally and functionally provides an evolutionary link between glycoside hydrolase enzyme families 13 and 70. Applied and Environmental Microbiology, 77(22), 81548163. https://doi.org/10.1128/AEM.05735-11

Lee, B.-H., Yan, L., Phillips, R. J., Reuhs, B. L., Jones, K., Rose, D. R., Nichols, B. L., QuezadaCalvillo, R., Yoo, S. H. \& Hamaker, B. R. (2013). Enzyme-Synthesized Highly Branched Maltodextrins Have Slow Glucose Generation at the Mucosal $\alpha$-Glucosidase Level and Are Slowly Digestible In Vivo. PLoS ONE, 8(4), e59745. https://doi.org/10.1371/journal.pone.0059745

Leemhuis, H., Dobruchowska, J. M., Ebbelaar, M., Faber, F., Buwalda, P. L., Van Der Maarel, M. J. E. C., Kamerling, J. P., \& Dijkhuizen, L. (2014). Isomalto/malto-polysaccharide, a novel soluble dietary fiber made via enzymatic conversion of starch. Journal of Agricultural and Food Chemistry, 62(49), 12034-12044. https://doi.org/10.1021/jf503970a

Leman, P., Goesaert, H., Vandeputte, G. E., Lagrain, B., \& Delcour, J. A. (2005). Maltogenic amylase has a non-typical impact on the molecular and rheological properties of starch. Carbohydrate Polymers, 62(3), 205-213. https://doi.org/10.1016/j.carbpol.2005.02.023

Nishiyama, Y. (2009). Structure and properties of the cellulose microfibril. Journal of Wood Science, 55(4), 241-249. https://doi.org/10.1007/s10086-009-1029-1

Pérez, S., \& Bertoft, E. (2010). The molecular structures of starch components and their contribution to the architecture of starch granules: A comprehensive review. Starch - Stärke, 62. https://doi.org/10.1002/star.201000013

Pettolino, F. A., Walsh, C., Fincher, G. B., \& Bacic, A. (2012). Determining the polysaccharide composition of plant cell walls. Nature Protocols, 7(9), 1590-1607. https://doi.org/10.1038/nprot.2012.081

Pfannemüller, B. (1987). Influence of chain length of short monodisperse amyloses on the formation of A- and B-type X-ray diffraction patterns. International Journal of Biological Macromolecules, 9(2), 105-108. https://doi.org/10.1016/0141-8130(87)90034-1

Raigond, P., Ezekiel, R., \& Raigond, B. (2015). Resistant starch in food: A review. Journal of the Science of Food and Agriculture, 95(10), 1968-1978. https://doi.org/10.1002/jsfa.6966

Sorndech, W., Meier, S., Jansson, A. M., Sagnelli, D., Hindsgaul, O., Tongta, S., \& Blennow, A. (2015). Synergistic amylomaltase and branching enzyme catalysis to suppress cassava starch digestibility. Carbohydrate Polymers, 132, 409-418. https://doi.org/10.1016/j.carbpol.2015.05.084

Suzuki, R., Koide, K., Hayashi, M., Suzuki, T., Sawada, T., Ohdan, T., Takahashi, H., Nakamura, Y., Fujita, N., \& Suzuki, E. (2015). Functional characterization of three (GH13) branching enzymes involved in cyanobacterial starch biosynthesis from Cyanobacterium sp. NBRC 102756. Biochimica et Biophysica Acta - Proteins and Proteomics, 1854(5), 476-484. https://doi.org/10.1016/j.bbapap.2015.02.012

Takeda, Y., Tomooka, S., \& Hizukuri, S. (1993). Structures of branched and linear molecules of rice amylose. Carbohydrate Research, 246(1), 267-272. https://doi.org/10.1016/00086215(93)84038-8

Tomasik, P., \& Schilling, C. H. (2004). Chemical modification of starch. Advances in Carbohydrate Chemistry and Biochemistry, 59(4), 175-403. https://doi.org/10.1016/s0065-2318(04)59005-4 
van der Maarel, M., \& Leemhuis, H. (2013). Starch modification with microbial $\alpha$-glucanotransferase enzymes. Carbohydrate Polymers, 93(1), 116-121. https://doi.org/10.1016/j.carbpol.2012.01.065

van der Maarel, M., van der Veen, B., Uitdehaag, J., Leemhuis, H., \& Dijkhuizen, L. (2002). Properties and applications of starch-converting enzymes of the $\alpha$-amylase family. Journal of Biotechnology, 94(2), 137-155. https://doi.org/10.1016/S0168-1656(01)00407-2

van Leeuwen, S. S., Kralj, S., van Geel-Schutten, I. H., Gerwig, G. J., Dijkhuizen, L., \& Kamerling, J. P. (2008). Structural analysis of the $\alpha$-d-glucan (EPS180) produced by the Lactobacillus reuteri strain 180 glucansucrase GTF180 enzyme. Carbohydrate Research, 343(7), 1237-1250. https://doi.org/10.1016/j.carres.2008.01.042

van Leeuwen, S. S., Leeflang, B. R., Gerwig, G. J., \& Kamerling, J. P. (2008). Development of a ${ }^{1}$ H NMR structural-reporter-group concept for the primary structural characterisation of $\alpha$-dglucans. Carbohydrate Research, 343(6), 1114-1119. https://doi.org/10.1016/j.carres.2008.01.043

Xu, Y., Zhou, X., Bai, Y., Wang, J., Wu, C., Xu, X., \& Jin, Z. (2014). Cycloamylose production from amylomaize by isoamylase and Thermus aquaticus 4- $\alpha$-glucanotransferase. Carbohydrate Polymers, 102, 66-73. https://doi.org/10.1016/j.carbpol.2013.10.065

Zhao, J., Schols, H. A., Chen, Z., Jin, Z., Buwalda, P., \& Gruppen, H. (2015). Distribution of phosphorus and hydroxypropyl groups within granules of modified sweet potato starches as determined after chemical peeling. Carbohydrate Polymers, 132, 630-637.

https://doi.org/10.1016/j.carbpol.2015.06.078 


\section{Supplementary information}

7.1 IMMP synthesis yield

\begin{tabular}{|l|c|}
\hline \multicolumn{1}{|c|}{ Sample } & $\begin{array}{c}\text { Synthesis yield } \\
\text { \% (w/w) }\end{array}$ \\
\hline Potato IMMP & 75.1 \\
\hline Waxy Potato IMMP & 77.2 \\
\hline Maize IMMP & 80.6 \\
\hline Waxy Maize IMMP & 77.7 \\
\hline Rice IMMP & 91.6 \\
\hline Waxy Rice IMMP & 94.1 \\
\hline Sweet Potato IMMP & 84.6 \\
\hline Wheat IMMP & 90.3 \\
\hline
\end{tabular}


7.2 Elution of starches and their respective IMPs on GPC-MALLS (DMSO-elution)
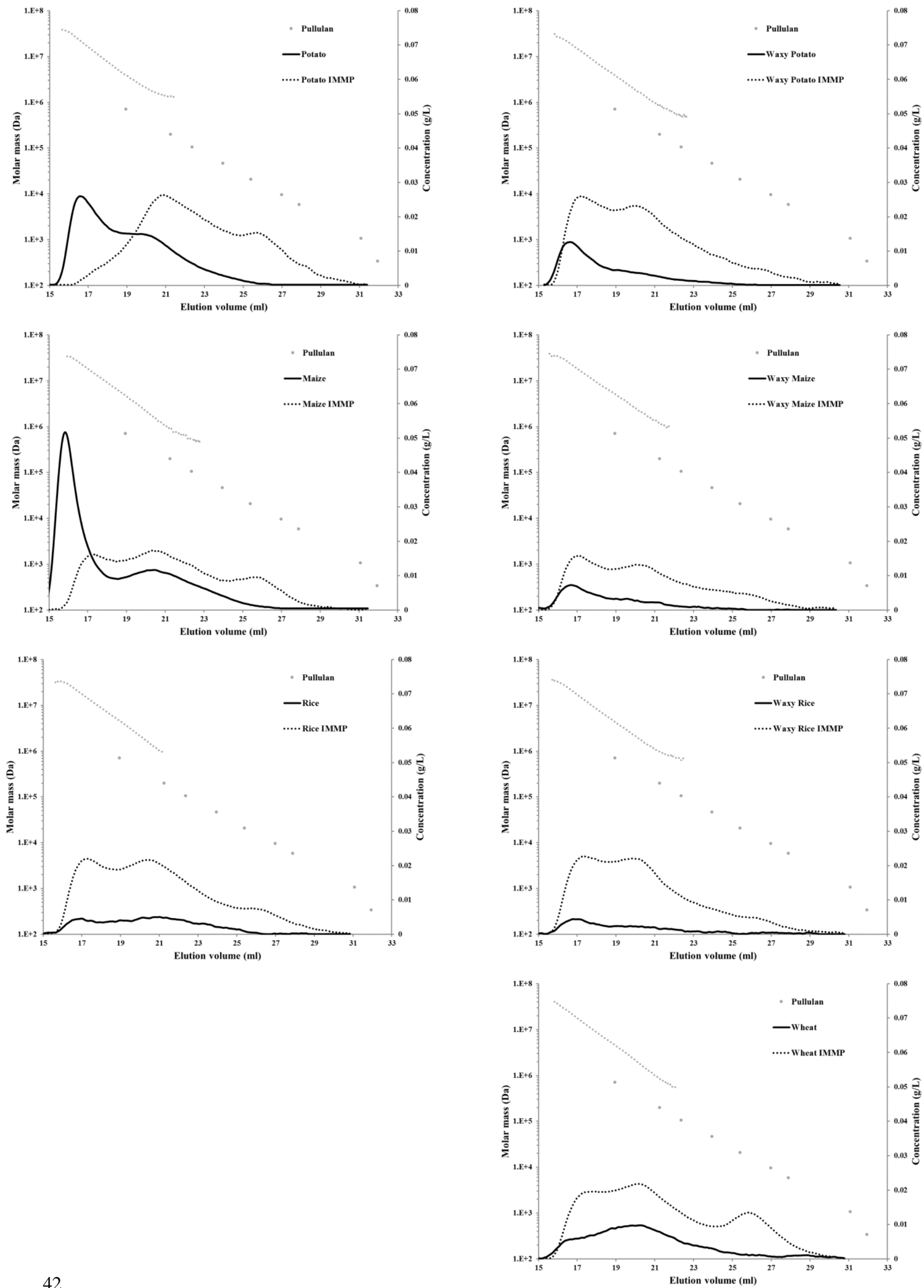
7.3 SEC-RI fractionation yield and $\alpha-(1 \rightarrow 6)$ content of IMMPs and their respective fractions.

\begin{tabular}{|c|c|c|c|c|}
\hline IMMP & Fraction & $\begin{array}{c}\%(w / w) \\
\text { fractionation yield }\end{array}$ & $\begin{array}{c}\text { Measured } \\
\alpha-(1 \rightarrow 6) \% \\
{ }^{1} \text { H NMR }\end{array}$ & $\begin{array}{l}\text { Weighed } \\
\alpha-(1 \rightarrow 6) \text { content } \\
\text { per fraction and } \\
\text { recalculated total }\end{array}$ \\
\hline Potato & $\begin{array}{c}\text { Total } \\
\text { HMW } \\
\text { MMW } \\
\text { LMW }\end{array}$ & $\begin{array}{c}\mathbf{5 5 . 6} \\
30.4 \\
6.1 \\
19.0\end{array}$ & $\begin{array}{c}24.6 \\
8.9 \\
13.6 \\
52.1\end{array}$ & $\begin{array}{l}\frac{24.2}{4.9} \\
1.5 \\
17.8\end{array}$ \\
\hline Waxy Potato & $\begin{array}{c}\text { Total } \\
\text { HMW } \\
\text { MMW } \\
\text { LMW } \\
\text { LMW II }\end{array}$ & $\begin{array}{c}\mathbf{7 3 . 9} \\
57.2 \\
5.1 \\
4.7 \\
7.0\end{array}$ & $\begin{array}{l}4.9 \\
4.7 \\
4.5 \\
4.5 \\
9.1\end{array}$ & $\begin{array}{l}\underline{5.1} \\
3.6 \\
0.3 \\
0.3 \\
0.9\end{array}$ \\
\hline Maize & $\begin{array}{c}\text { Total } \\
\text { HMW } \\
\text { MMW } \\
\text { LMW }\end{array}$ & $\begin{array}{c}\mathbf{4 9 . 6} \\
19.8 \\
9.9 \\
19.9 \\
\end{array}$ & $\begin{array}{c}19.0 \\
6.8 \\
10.3 \\
54.5 \\
\end{array}$ & $\begin{array}{c}\underline{26.6} \\
2.7 \\
2.1 \\
21.9\end{array}$ \\
\hline Waxy Maize & $\begin{array}{l}\text { Total } \\
\text { HMW } \\
\text { MMW } \\
\text { LMW }\end{array}$ & $\begin{array}{c}\mathbf{6 2 . 2} \\
40.9 \\
13.1 \\
8.2\end{array}$ & $\begin{array}{l}6.2 \\
5.3 \\
6.0 \\
9.9\end{array}$ & $\begin{array}{l}\frac{6.1}{3.5} \\
1.3 \\
1.3\end{array}$ \\
\hline Rice (II) & $\begin{array}{l}\text { Total } \\
\text { HMW } \\
\text { MMW } \\
\text { LMW }\end{array}$ & $\begin{array}{l}\mathbf{5 9 . 4} \\
33.3 \\
14.1 \\
11.9\end{array}$ & $\begin{array}{c}12.8 \\
6.2 \\
8.4 \\
28.8\end{array}$ & $\begin{array}{l}\frac{11.3}{3.5} \\
2.0 \\
5.8\end{array}$ \\
\hline Waxy Rice & $\begin{array}{c}\text { Total } \\
\text { HMW } \\
\text { MMW } \\
\text { LMW }\end{array}$ & $\begin{array}{l}\mathbf{6 9 . 0} \\
38.2 \\
19.5 \\
11.3\end{array}$ & $\begin{array}{l}6.0 \\
5.5 \\
6.5 \\
5.6\end{array}$ & $\begin{array}{l}\frac{5.8}{3.0} \\
1.8 \\
0.9\end{array}$ \\
\hline Sweet Potato & $\begin{array}{l}\text { Total } \\
\text { HMW } \\
\text { MMW } \\
\text { LMW }\end{array}$ & \begin{tabular}{c|}
$\mathbf{6 6 . 1}$ \\
44.7 \\
6.3 \\
15.1 \\
\end{tabular} & $\begin{array}{c}18.4 \\
6.9 \\
10.4 \\
47.3\end{array}$ & $\begin{array}{l}\frac{16.4}{4.6} \\
1.0 \\
10.8\end{array}$ \\
\hline Wheat & $\begin{array}{l}\text { Total } \\
\text { HMW } \\
\text { MMW } \\
\text { LMW }\end{array}$ & $\begin{array}{l}\mathbf{5 6 . 5} \\
22.9 \\
14.0 \\
19.5\end{array}$ & $\begin{array}{c}23.3 \\
6.9 \\
8.4 \\
49.2\end{array}$ & $\begin{array}{l}\frac{21.9}{2.8} \\
2.1 \\
17.0\end{array}$ \\
\hline
\end{tabular}


7.4 Elution of IMMPs and their respective fractions on GPC-MALLS (DMSO-elution)
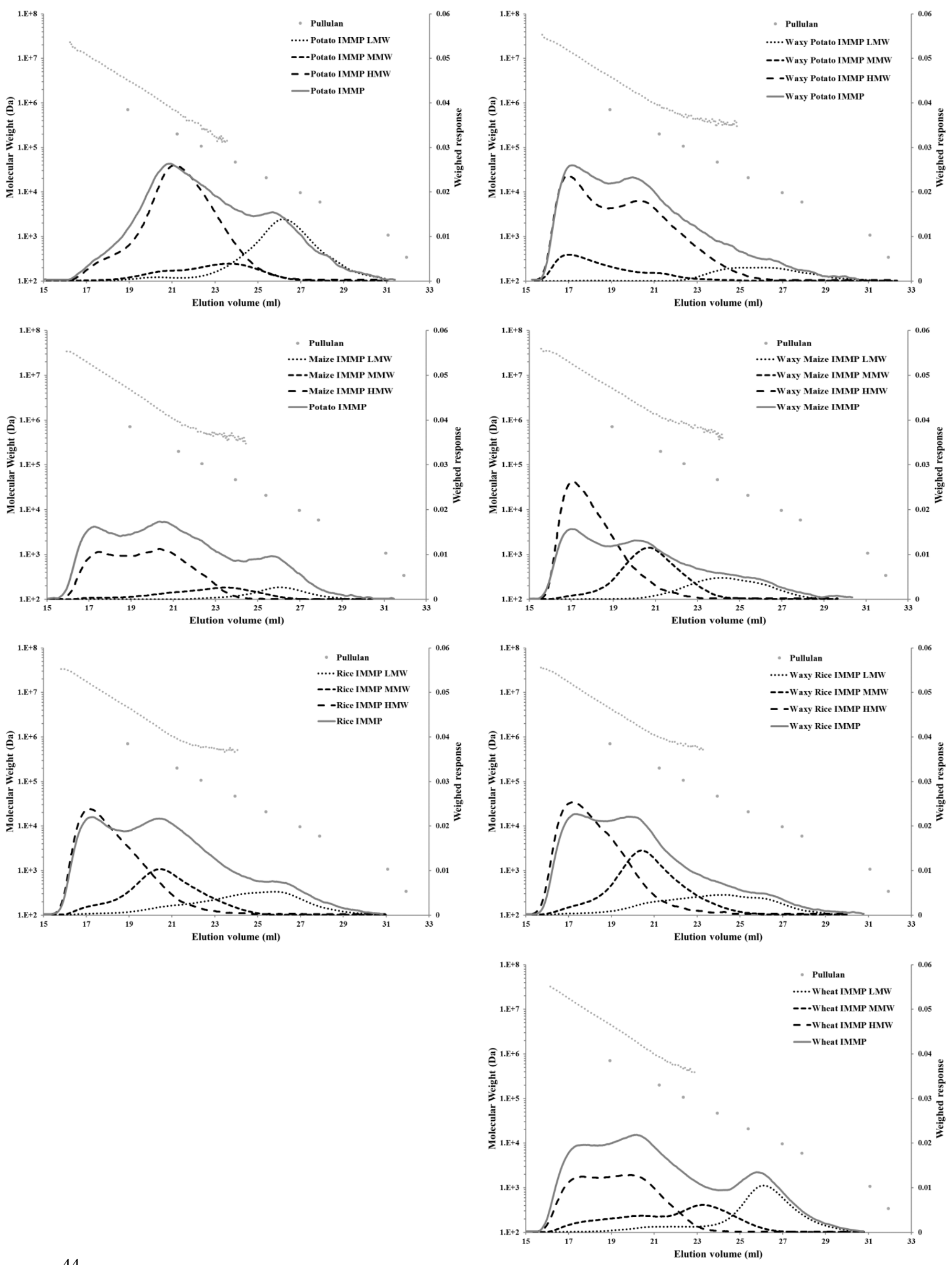


\begin{tabular}{|c|c|c|c|c|c|c|}
\hline & \multicolumn{5}{|c|}{ Methylation analysis (mol\%) } & \multirow{2}{*}{$\begin{array}{c}{ }^{1} \text { H NMR } \\
\alpha-(1 \rightarrow 6) \%\end{array}$} \\
\hline & T-glucose & $\alpha-(1 \rightarrow 4)$ & $\alpha-(1 \rightarrow 6)$ & $\alpha-(1 \rightarrow 4,6)$ & total $\alpha-(1 \rightarrow 6)$ & \\
\hline Native Potato & 4 & 95 & 0 & 2 & 2 & ND \\
\hline Potato IMMP & 4 & 76 & 17 & 3 & 20 & 25 \\
\hline HMW & 5 & 92 & 2 & 1 & 4 & 9 \\
\hline MMW & 5 & 93 & 2 & 1 & 3 & 14 \\
\hline LMW & 7 & 70 & 23 & 1 & 24 & 52 \\
\hline Native Waxy Potato & 5 & 94 & trace & 2 & 2 & ND \\
\hline Waxy Potato IMMP & 4 & 93 & 1 & 2 & 2 & 5 \\
\hline HMW & 4 & 94 & trace & 1 & 1 & 5 \\
\hline $\mathrm{MMW}^{*}$ & 5 & 94 & trace & 2 & 2 & 5 \\
\hline $\mathrm{LMW}^{* *}$ & 5 & 88 & 5 & 2 & 7 & 9 \\
\hline Native Rice & 4 & 94 & 0 & 2 & 2 & ND \\
\hline Rice IMMP & 5 & 90 & 3 & 3 & 6 & 13 \\
\hline HMW & 6 & 91 & 1 & 2 & 3 & 6 \\
\hline MMW & 7 & 90 & 1 & 2 & 3 & 8 \\
\hline LMW & 7 & 83 & 8 & 2 & 10 & 29 \\
\hline Native Waxy Rice & 5 & 91 & trace & 4 & 4 & ND \\
\hline Waxy Rice IMMP & 6 & 92 & trace & 2 & 2 & 6 \\
\hline HMW & 6 & 91 & trace & 3 & 3 & 6 \\
\hline MMW & 5 & 90 & 1 & 4 & 5 & 7 \\
\hline LMW & 7 & 90 & 1 & 2 & 3 & 6 \\
\hline Native Sweet Potato & 3 & 94 & trace & 2 & 2 & ND \\
\hline Sweet Potato IMMP & 4 & 87 & 6 & 2 & 8 & 18 \\
\hline HMW & 5 & 92 & 1 & 2 & 3 & 7 \\
\hline MMW & 7 & 89 & 3 & 2 & 5 & 10 \\
\hline LMW & 4 & 70 & 24 & 2 & 26 & 47 \\
\hline Native Wheat & 4 & 94 & trace & 2 & 2 & ND \\
\hline Wheat IMMP & 5 & 89 & 5 & 2 & 7 & 23 \\
\hline HMW & 6 & 92 & 1 & 2 & 3 & 7 \\
\hline MMW & 5 & 90 & 2 & 2 & 4 & 8 \\
\hline LMW & 5 & 72 & 22 & 1 & 23 & 49 \\
\hline Native Maize & 3 & 95 & 0 & 2 & 2 & ND \\
\hline Maize IMMP I & - & - & - & - & - & 19 \\
\hline HMW & - & - & - & - & - & 7 \\
\hline MMW & - & - & - & - & - & 10 \\
\hline LMW & - & - & - & - & - & 55 \\
\hline Maize IMMP II & 4 & 90 & 3 & 2 & 5 & - \\
\hline HMW & 5 & 92 & 1 & 2 & 3 & - \\
\hline MMW & 4 & 89 & 5 & 2 & 7 & - \\
\hline LMW & 4 & 56 & 39 & 1 & 40 & - \\
\hline Native Waxy Maize & 5 & 92 & trace & 3 & 3 & ND \\
\hline Waxy Maize IMMP & 5 & 91 & 1 & 3 & 4 & 6 \\
\hline HMW & 6 & 92 & trace & 2 & 2 & 5 \\
\hline MMW & 7 & 91 & trace & 2 & 2 & 6 \\
\hline LMW & 6 & 88 & 3 & 3 & 6 & 10 \\
\hline
\end{tabular}

Trace indicates residues $<0.5 \mathrm{~mol} \%, \mathrm{ND}=$ not determined due to the low solubility $\left(\mathrm{D}_{2} \mathrm{O}\right)$ during ${ }^{1} \mathrm{H}$ NMR measurements. 



\section{Chapter 3}

\section{Enzymatic fingerprinting of isomalto/malto-polysaccharides}

In this study, we present an enzymatic fingerprinting method for the characterization of isomalto/malto-polysaccharides (IMMPs). IMMPs are produced by the modification of starch with the 4,6- $\alpha$-glucanotransferase (GTFB) enzyme and consist of $\alpha-(1 \rightarrow 4), \alpha-$ $(1 \rightarrow 6)$ and $\alpha-(1 \rightarrow 4,6)$ linked glucoses. Enzymes were used separately, simultaneously or in successive order to specifically degrade and/or reveal IMMP substructures. The enzymatic digests were subsequently analysed with HPSEC and HPAEC to reveal the chain length distribution (CLD) of different IMMP substructures. The presence of amylose in the substrate resulted in the formation of linear $\alpha-(1 \rightarrow 6)$ linked glycosidic chains $(13.5 \mathrm{kDa})$ in the former amylopectin fraction. The length of these chains indicates that GTFB transferase activity on amylopectin is more likely to elongate single amylopectin chains than to provide an even spread. Enzymatic fingerprinting also revealed that the GTFB enzyme is capable of introducing large $(20 \mathrm{kDa})$ linear $\alpha-(1 \rightarrow 6)$ linked glycosidic chains in the $\alpha$-glucan substrate.

Based on: van der Zaal, P.H., Klostermann, C.E., Schols, H. A., Bitter J.H. \& Buwalda, P.L. (2018). Enzymatic fingerprinting of isomalto/malto-polysaccharides. Carbohydrate Polymers. https://doi.org/10.1016/j.carbpol.2018.09.049 


\section{Introduction}

In-depth characterization of polysaccharides is essential for the understanding of their physicochemical properties. Structural information on monosaccharide composition and the glycosidic linkages present can be measured by permethylation, FTIR and NMR analysis (Fontana, Li, Yang, \& Widmalm, 2015; Petersen, Motawie, Møller, Hindsgaul, \& Meier, 2015; Pettolino, Walsh, Fincher, \& Bacic, 2012; Siddiqui, Aman, Silipo, Qader, \& Molinaro, 2014; van der Zaal, Schols, Bitter, \& Buwalda, 2017). Structural information on the size and hydrodynamic volume of a polysaccharide can be measured with light scattering and chromatographic methods (Bourgoin, Zablackis, \& Poli, 2008; Pérez et al., 2011). The above-mentioned methods are often combined, to create a more detailed picture of the investigated polysaccharide (Irague, Tarquis, Doublier, Moulis, \& Monsan, 2012; Song \& Du, 2012; Synytsya \& Novak, 2014; Wang, Zhao, Tian, Yang, \& Yang, 2015). However, polysaccharide substructures such as the chain length distribution (CLD) and repeating patterns often remain challenging to reveal.

The substructure of starch can be analysed chemically, physically and enzymatically. Chemically, with the classic iodine staining method (Shen, Bertoft, Zhang, \& Hamaker, 2013) or with more sophisticated methods such as the quantification of long branches in starch amylopectin with hydrophobic probes that 'recognize' $\alpha-(1 \rightarrow 4)$ glycosidic helix structures and change fluorescence when docked (Beeren \& Hindsgaul, 2014). Physically, non-invasive methods such as X-ray and neutron diffraction are also used for the study of small structural elements and the amount/type of crystallinity present in polysaccharides (Blazek \& Gilbert, 2011). However, these chemical and physical methods cannot compete with the pattern recognition that is displayed in nature. Enzymes are by far, the most sophisticated tools available for pattern recognition in polysaccharide molecules (Bai, Gangoiti, Dijkstra, Dijkhuizen, \& Pijning, 2016; Tanackovic et al., 2016). Enzymes are therefore often used instead of, or in combination with, traditional methods for the characterization of polysaccharides.

Using the pattern recognizing abilities of enzymes as a tool for characterization is also called enzymatic fingerprinting. Heteropolysaccharides, such as pectin, are often characterized in this way (Schols \& Voragen, 1996; Voragen, Coenen, Verhoef, \& Schols, 2009), the selected enzymatic toolbox used for pectin analysis is able to hydrolyse specific structural components that are hard to locate and detect otherwise (Broxterman, Picouet, \& Schols, 2017; Ognyanov et al., 2016; Remoroza, Broxterman, Gruppen, \& Schols, 2014; Remoroza, Buchholt, Gruppen, \& Schols, 2014). Enzymatic fingerprinting can also be used for the characterization and identification of other 
complex heteropolysaccharides such as locust bean gum, xyloglucans and arabinoxylans (Grün et al., 2015; Ray, Vigouroux, Quémener, Bonnin, \& Lahaye, 2014; Tian, Gruppen, \& Schols, 2015). The above-mentioned fingerprinting methods show that an effective enzymatic toolbox has to be optimized towards its substrate, due to the high specificity of its enzymes.

Starches and other $\alpha$-glucans are often characterized with CLD analysis (Hizukuri, Kaneko, \& Takeda, 1983; Pérez \& Bertoft, 2010; Vilaplana \& Gilbert, 2010). Where pullulanase (Kajiura, Takata, Kuriki, \& Kitamura, 2010; Lee et al., 2013; Lian, Kang, Sun, Liu, \& Li, 2015), or isoamylase (Ciric, Woortman, \& Loos, 2014; Grewal et al., 2015; Rolland-Sabaté et al., 2013; Sorndech et al., 2016) are used as debranching enzymes for the specific hydrolysis of the $\alpha$ - $(1 \rightarrow 6)$ glycosidic linkage from $\alpha$-glucan $\alpha$ $(1 \rightarrow 4,6)$ branching points. After debranching, the material is investigated with chromatographic methods, such as high performance anion exchange chromatography (HPAEC), where the CLD of the investigated $\alpha$-glucan is revealed. Variations in CLD can influence double helix formation (Gidley \& Bulpin, 1989), crystal structures and physical properties of $\alpha$-glucans (Alting et al., 2009; Gidley \& Bulpin, 1989; Kim, Kim, Moon, \& Choi, 2014). The use of enzymes in $\alpha$-glucan analysis has also helped to create a better understanding of starch granule architecture (Pérez \& Bertoft, 2010).

In this study, we present and implement an enzymatic fingerprinting method dedicated to the characterization of isomalto/malto-polysaccharides (IMMPs), with an enzymatic toolbox specialized to deal with $\alpha-(1 \rightarrow 4), \alpha-(1 \rightarrow 6)$ and $\alpha-(1 \rightarrow 4,6)$ linked glucoses. IMMPs are $\alpha$-glucans produced by the enzymatic modification of starch with a $4,6-\alpha-$ glucanotransferase enzyme (Leemhuis et al., 2014). The structure of IMMPs has been characterized (Dobruchowska et al., 2012; Leemhuis et al., 2014; van der Zaal et al., 2017), but detailed information on IMMP substructure, such as the CLD of the newly incorporated $\alpha-(1 \rightarrow 6)$ linked glycosidic chains and the CLD of their $\alpha-(1 \rightarrow 4)$ linked glycosidic acceptor chains has remained unknown, until now. With the use of pure and specific $\alpha$-glucan degrading enzymes, enzymatic fingerprinting was used to reveal the CLD of IMMP substructures, a feat that is less likely to be achieved with traditional means of analysis. 


\section{Materials \& methods}

\subsection{Materials}

Potato starch, waxy potato starch (Eliane 100), and amylomaltase treated potato starch (ATPS, Etenia 457) were provided by AVEBE (Veendam, the Netherlands). Potato starch IMMP and waxy potato IMMP were produced from the abovementioned starches by incubation with a N-terminal truncated 4,6- $\alpha$-glucanotransferase (GTFB- $\Delta \mathrm{N}$ ) enzyme as described in van der Zaal et al. (2017). The potato IMMP high molecular weight (HMW) fraction and the potato IMMP low molecular weight (LMW) fraction were produced by preparative fractionation of potato IMMP into the respective HMW and LMW fractions, described previously in van der Zaal et al. (2017). Dextran ( 35 $\mathrm{kDa}$ ), glucose, maltotriose and isomaltotriose were obtained from Sigma-Aldrich (St. Louis, MO, USA), pullulan was purchased from Hayashibara (Japan). Isomaltose was obtained from Megazyme (Bray, Wicklow, Ireland) and maltose was obtained from Fluka (Buchs, Switzerland). Isoamylase (EC 3.2.1.68) (Pseudomonas sp.), isopullulanase (EC 3.2.1.57) (Aspergillus niger) and $\beta$-amylase (EC 3.2.1.2) (Barley) were purchased from Megazyme. Dextranase (EC 3.2.1.11) (Chaetomium erraticum) was obtained from Sigma-Aldrich (St. Louis, MO, USA).

\subsection{Glucanohydrolase purity and specificity}

ATPS, pullulan and dextran were used as a set of model substrates to investigate the purity and specificity of a range of glucanohydrolases. ATPS, pullulan and dextran were dissolved at concentration of $2.5 \mathrm{mg} / \mathrm{mL}$ in an acetate buffer $(20 \mathrm{mM}, \mathrm{pH}=5.0,5 \mathrm{mM}$ $\mathrm{CaCl}_{2}$ ) by autoclaving at $121{ }^{\circ} \mathrm{C}$ for $15 \mathrm{~min}$. The enzymes were added after the solutions were cooled to $40{ }^{\circ} \mathrm{C}$ for a subsequent incubation at $40{ }^{\circ} \mathrm{C}$ (or $39{ }^{\circ} \mathrm{C}$, in the case of isopullulanase) and $100 \mathrm{rpm}$ in a Climo-shaker IFF1-X (Kuhner, Bisfelden, Switzerland) for 1.5 and $24 \mathrm{~h}$. Isoamylase, $\beta$-amylase and isopullulanase were added at a concentration of $0.16 \mathrm{U} / \mathrm{mg}$ substrate (An et al., 2011; Chen et al., 2004; Huijbrechts et al., 2007; Leemhuis et al., 2014) and dextranase at a concentration of $0.052 \mathrm{U} / \mathrm{mg}$ substrate (Gu et al., 2018). The enzymes were inactivated at $95{ }^{\circ} \mathrm{C}$ and $300 \mathrm{rpm}$ for 10 min using a ThermoMixer (Eppendorf, Hamburg, Germany). The inactivated enzymatic digests were stored at room temperature to avoid crystallisation and analysed within 3 days after preparation. The purity and specifity was evaluated with HPSEC (§2.4) and HPAEC (§2.5). The selected glucanohydrolases, listed above, were found to be suitable for enzymatic fingerprinting at the abovementioned concentrations. 


\subsection{Enzymatic fingerprinting}

The above-mentioned glucanohydrolases were used to create an enzymatic toolbox tailored towards IMMP characterization (Figure 1). Isoamylase specifically targets $\alpha$ $(1 \rightarrow 4,6)$ branching points and is often used for determining the CLD in starches (Ciric et al., 2014; Grewal et al., 2015; Sorndech et al., 2016). Isoamylase was preferred over pullulanase, since isoamylase displayed a lower degree of unwanted hydrolysis (e.g. lower maltose and maltotriose contents) compared to pullulanase after debranching ATPS (unpublished results). $\beta$-amylase is an exo-acting enzyme that hydrolyses linear $\alpha-(1 \rightarrow 4)$ glycosidic linkages into maltose (Goesaert, Bijttebier, \& Delcour, 2010; Witt \& Gilbert, 2014), the exo-activity allows this enzyme to be deployed strategically, before and after hydrolysis with other enzymes. Dextranase is an endo-acting enzyme that hydrolyses $\alpha-(1 \rightarrow 6)$ glycosidic linkages of linear $\alpha-(1 \rightarrow 6)$ glucans into small oligosaccharides and isopullulanase is able to specifically hydrolyse an $\alpha-(1 \rightarrow 4)$ glycosidic linkage situated on the reducing side of a linear $\alpha-(1 \rightarrow 6)$ glycosidic linkage (Khalikova, Susi, \& Korpela, 2005).

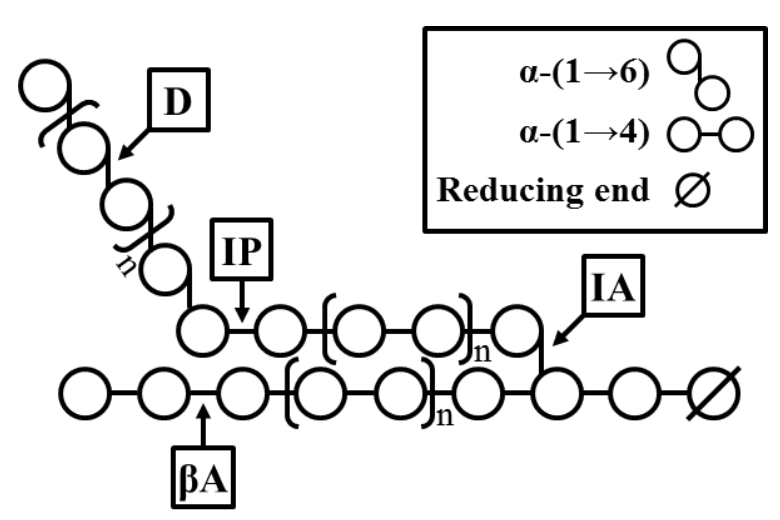

Figure 6. Activities of the different enzymes in the enzymatic toolbox for IMMP characterization; isoamylase (IA), $\beta$-amylase ( $\mathbf{\beta A})$, dextranase (D), and isopullulanase (IP).

Potato IMMP, waxy potato IMMP and potato IMMP HMW were dissolved in an acetate buffer $\left(20 \mathrm{mM}, \mathrm{pH}=5.0,5 \mathrm{mM} \mathrm{CaCl}_{2}\right)$ at a concentration of $2.5 \mathrm{mg} / \mathrm{mL}$, heated to $95^{\circ} \mathrm{C}$ for $15 \mathrm{~min}$ and subsequently cooled to $39^{\circ} \mathrm{C}$. Isoamylase, $\beta$-amylase and isopullulanase were added at concentrations of $0.16 \mathrm{U} / \mathrm{mg}$ substrate and dextranase was added at a concentration of $0.052 \mathrm{U} / \mathrm{mg}$ substrate. The potato IMMP LMW fraction was treated differently due to the low amount of sample, the above mentioned enzymes were added in a concentration of $0.4 \mathrm{U} / \mathrm{mg}$ and dextranase was added in a concentration of 0.13 $\mathrm{U} / \mathrm{mg}$. An overview of the different incubations is displayed in Figure 2, all incubations were done at $39{ }^{\circ} \mathrm{C}$ and $100 \mathrm{rpm}$ for $4 \mathrm{~h}$, subsequent inactivation was done at $95{ }^{\circ} \mathrm{C}$ for 15 min unless stated otherwise. Individual incubation with isoamylase (Figure 2, II), one-pot incubation with isoamylase and $\beta$-amylase (Figure 2, III), one-pot incubation 
with isoamylase, $\beta$-amylase and dextranase (Figure 2, IV), one-pot incubation with isoamylase, $\beta$-amylase and dextranase followed by inactivation $\left(95^{\circ} \mathrm{C}, 15 \mathrm{~min}\right)$ and subsequent isopullulanase incubation (3h) (Figure 2, V) and a one-pot incubation with isoamylase, $\beta$-amylase and isopullulanase (Figure 2 , VI). $\alpha-(1 \rightarrow 6)$ and $\alpha-(1 \rightarrow 4)$ linked glycosidic reference oligosaccharides were prepared in a similar way by incubating dextran $(2.5 \mathrm{mg} / \mathrm{mL})$ with dextranase $(0.052 \mathrm{U} / \mathrm{mg})$ and by incubating ATPS (2.5 $\mathrm{mg} / \mathrm{mL})$ with isoamylase $(0.16 \mathrm{U} / \mathrm{mg}$ ) respectively (supplementary information 7.1 ).

\subsection{Monitoring enzyme action with HPSEC-RI}

Sample solutions $(2.5 \mathrm{mg} / \mathrm{mL})$ were centrifuged at $7300 \mathrm{x} \mathrm{g}$ and $20{ }^{\circ} \mathrm{C}$ for $10 \mathrm{~min}$ and the supernatant was used for HPSEC analysis. An Ultimate 3000 HPLC (Dionex, Sunnyvale, USA) was used with a column set which consisted of three in series connected TosoHaas (Tokyo, Japan) TSK-Gel columns (4000PWXL-3000PWXL2500PWXL), (6 x $150 \mathrm{~mm}$ ), with a guard column and a Shodex type RI-101 refractive index detector (Showa Denko, K.K., Kawasaki, Japan). With 0.2 M NaNO3 as eluent and a flow of $0.6 \mathrm{~mL} / \mathrm{min}$ at $55^{\circ} \mathrm{C}$. A volume of $10 \mu \mathrm{L}$ sample solution was injected onto the column. A pullulan standard series (180 - 780000 Da) (Fluka) was used for calibration. The buffer and all enzymes were run separately as controls. Data analysis was performed with ChromeleonTM 7.1 software from Thermo Fisher Scientific (Waltham, Massachusetts, USA).

\subsection{Monitoring enzyme action with HPAEC-PAD}

Sample solutions were diluted to $0.5 \mathrm{mg} / \mathrm{mL}$ with Millipore water and centrifuged at $7300 \mathrm{x} \mathrm{g}$ and $20{ }^{\circ} \mathrm{C}$ for $10 \mathrm{~min}$, the supernatant was used for HPAEC analysis. The analysis was performed on an ICS5000 High Performance Anion Exchange Chromatography system with Pulsed Amperometric Detection (HPAEC-PAD) (Dionex Corporation, Sunnyvale, USA) equipped with a CarboPac PA-1 column ( 2 x $250 \mathrm{~mm}$ ) and a CarboPac PA-1 guard column ( $2 \times 25 \mathrm{~mm}$ ). The two mobile phases were (A) 0.1 $\mathrm{M} \mathrm{NaOH}$ and (B) $1 \mathrm{M} \mathrm{NaOAc}$ in $0.1 \mathrm{M} \mathrm{NaOH}$ and the flow rate was set to $0.3 \mathrm{~mL} / \mathrm{min}$. Two gradients were used with these mobile phases. Gradient 1 (section 2.2); 0-50 min $5-40 \% \mathrm{~B}, 50-65 \min 40-100 \% \mathrm{~B}, 65-70 \mathrm{~min} 100 \% \mathrm{~B}$ ending with a 70-85 min reequilibration at $5 \%$ B. Gradient 2 (section 2.3); 0-50 min 5-30\% B, 50-65 min 30-100\% $\mathrm{B}, 65-70$ min $100 \% \mathrm{~B}$ ending with a $70-85$ min re-equilibration at 5\% B. An injection volume of $10 \mu \mathrm{L}$ sample solution was injected onto the column. Glucose, maltose, maltotriose, isomaltose and isomaltotriose $(10-100 \mu \mathrm{g} / \mathrm{mL})$ were run as standards. Data analysis was performed with ChromeleonTM 7.1 software Thermo Fisher Scientific (Waltham, Massachusetts, USA). 


\section{Results \& Discussion}

\subsection{IMMP structure}

Previous research on IMMP structure indicated that the substructure of an average IMMP can be divided into three main groups (van der Zaal et al., 2017); linear $\alpha-(1 \rightarrow 6)$ linked glycosidic chains introduced by the GTFB- $\Delta$ N enzyme (Figure 2, red line), $\alpha$ $(1 \rightarrow 4)$ linked glycosidic chains shielded by linear $\alpha-(1 \rightarrow 6)$ linked glycosidic chains (Figure 2, black line) and unshielded $\alpha-(1 \rightarrow 4)$ linked glycosidic chains (Figure 2, green line). Until now, this structure could only be partly validated, since previously used methods were not able to provide insight into the chain length distribution (CLD) of these structures.

\subsection{Enzymatic fingerprinting method}

The enzymatic toolbox presented in this research was optimized for IMMP substructure analysis and is focused on the step-wise decomposition of IMMPs. It consists of four enzymes with specific activities; isoamylase, $\beta$-amylase, dextranase and isopullulanase (Figure 1 and \$2.3). These enzymes, were used separately, simultaneously or in successive order to specifically degrade and/or reveal IMMP substructures. The result is a step-wise decomposition of the substrate, in which every step generates diagnostic oligomers that relate to the substrate's original structure (Figure 2). The purity and specificity of these enzymes was tested and confirmed on model substrates (§2.3).

We will start by describing the enzymatic fingerprinting approach by discussing the step-wise decomposition of potato IMMP (Figure 2 and Figure 3). Subsequently we will discuss and compare the obtained structures with other IMMPs in chapter §3.3. Potato IMMP is used as an example since it contains all the (sub)structures and linkage types discussed above (\$3.1) (van der Zaal et al., 2017). Figure 3 depicts the corresponding HPSEC and HPSAEC chromatograms per step for potato IMMP, the steps in Figures 2 and 3 follow a consistent colour scheme.

\subsubsection{Isoamylase debranching}

Potato IMMP was firstly debranched with isoamylase (Figure 2 (II) and Figure 3 (II)), in order to investigate its CLD. The debranching of potato IMMP caused a big drop in molecular weight $\left(\mathrm{M}_{\mathrm{w}}\right)$ visible in HPSEC (Figure 3 (II)), indicating that most of the larger structures present in potato IMMP are rich in $\alpha-(1 \rightarrow 4,6)$ branching points. Isoamylase treatment resulted in two main structural groups; $\alpha-(1 \rightarrow 4)$ linked glycosidic chains shielded by $\alpha-(1 \rightarrow 6)$ linked glycosidic chains (black line) and unshielded $\alpha$ - 
$(1 \rightarrow 4)$ linked glycosidic chains (green line) (Figure 2 (II)). The unshielded $\alpha-(1 \rightarrow 4)$ linked glycosidic chains are clearly visible in the HPAEC chromatogram and correlate with the $\alpha-(1 \rightarrow 4)$ linked glycosidic reference oligosaccharides (Figure 3 (II), supplementary information 7.1). The unshielded $\alpha-(1 \rightarrow 4)$ linked glycosidic chains are distinguishable up to DP $\sim 30$ at $\mathrm{t}_{\mathrm{el}}=50 \mathrm{~min}$. (Figure 3 (II)). From reference patterns it is clear that $\alpha-(1 \rightarrow 6)$ linked glycosidic oligomers are eluting much quicker than $\alpha$ $(1 \rightarrow 4)$ linked glycosidic oligomers, with the lump at 20-35 min (Figure 3 (I, II, III, VI)) representing unresolved DPs of $\alpha-(1 \rightarrow 6)$ linked glycosidic oligomers (supplementary information 7.1). 


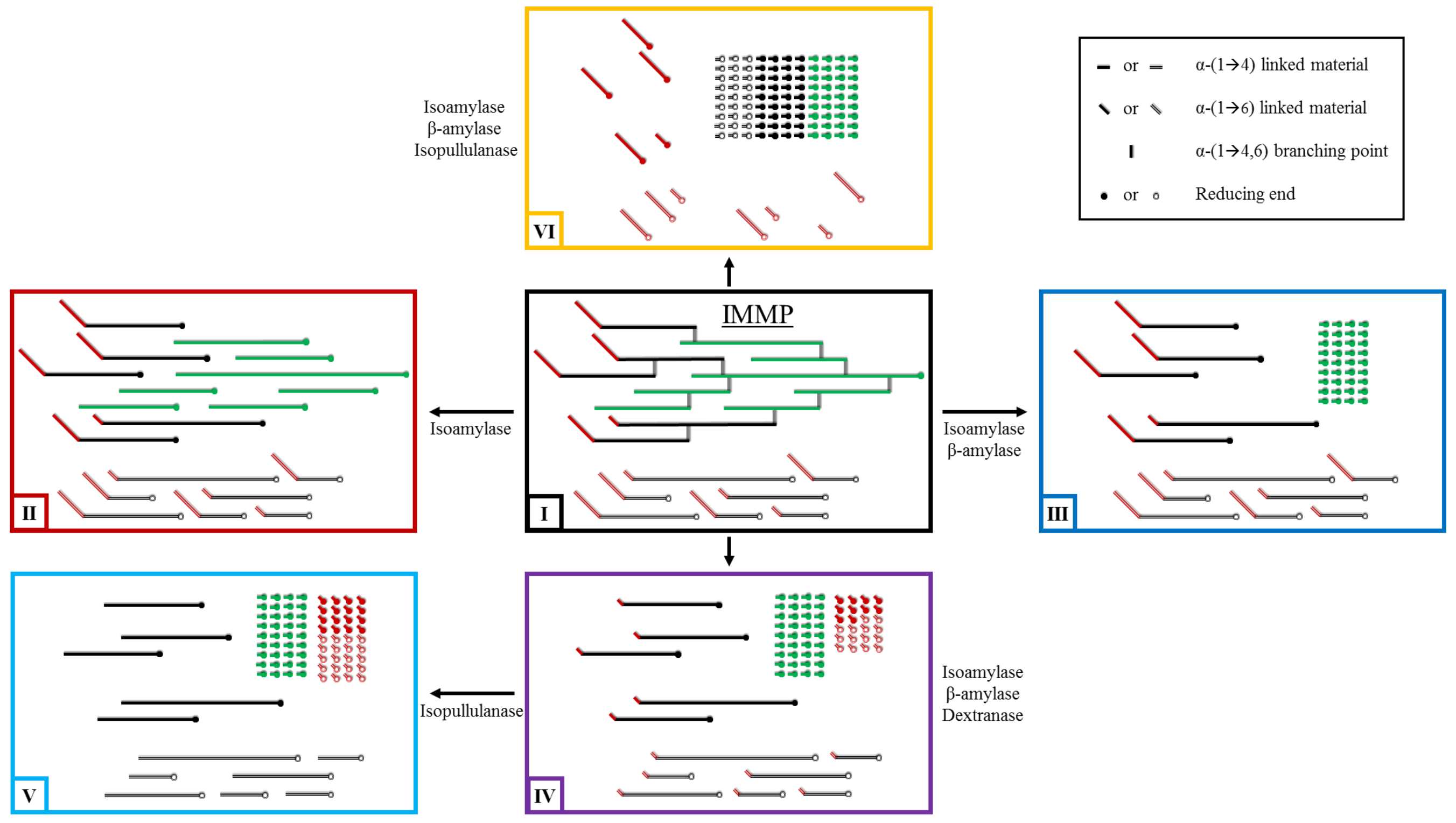

Figure 2. IMMP substructure analysis by enzymatic fingerprinting; GTFB- $\Delta$ N introduced $\alpha-(1 \rightarrow 6)$ linked glycosidic linkages (red line), shielded $\alpha-(1 \rightarrow 4)$ linked glycosidic linkages (black line) and unshielded $\alpha-(1 \rightarrow 4)$ linked glycosidic linkages (green line). Branched IMMPs and degradation products are represented with solid lines. Linear IMMPs and degradation products are represented with hollow lines. Enzymes are always inactivated before going to the next step. 


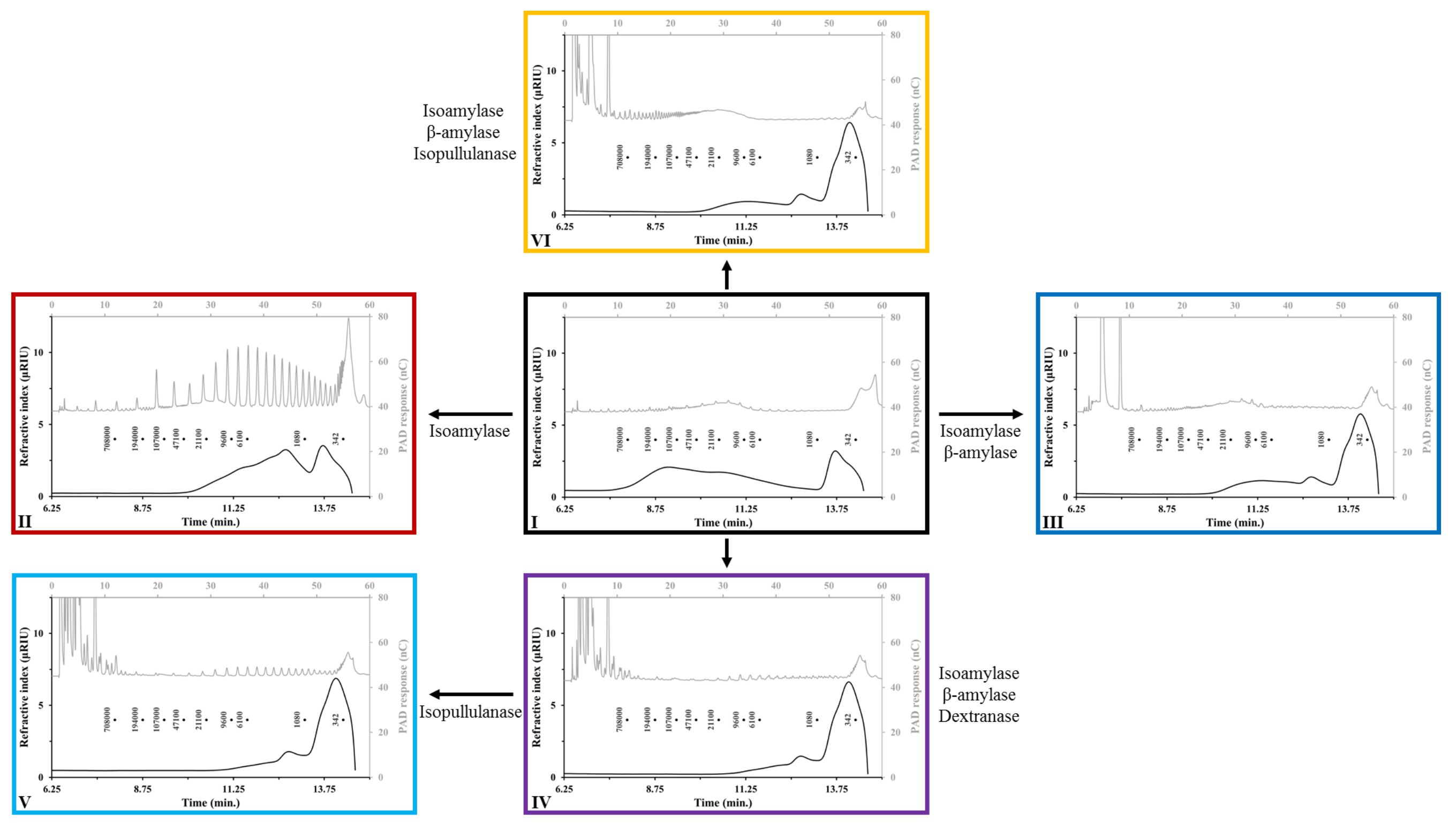

Figure 3. HPSEC (A) and HPAEC (B) profiles of potato IMMP, hydrolysed by isoamylase (II), one-pot incubation of isoamylase and $\beta$-amylase (III), one-pot incubation of isoamylase, $\beta$-amylase and dextranase (IV), one-pot incubation of isoamylase, $\beta$-amylase and dextranase followed by isopullulanase (V) and a one-pot incubation of isoamylase, $\beta$-amylase and isopullulanase (VI). 


\subsection{2 $\beta$-amylase and hybrid IMMP molecules}

The $\alpha-(1 \rightarrow 4)$ linked glycosidic chains free from linear $\alpha-(1 \rightarrow 6)$ linked glycosidic linkages contain an exposed non-reducing end that is easily degraded with $\beta$-amylase (green, Figure 2). The combined activity of isoamylase and $\beta$-amylase (Figure 2 (III)), therefore, results in the hydrolysis of the unshielded $\alpha-(1 \rightarrow 4)$ linked glycosidic chains (green) into maltose. All the larger remaining structures consist of linear $\alpha-(1 \rightarrow 4)$ linked glycosidic chains shielded by linear $\alpha-(1 \rightarrow 6)$ linked glycosidic chains, from now on called hybrid molecules (Figure 4). However, the presence of purely linear $\alpha-(1 \rightarrow 6)$ linked glycosidic chains cannot be excluded, since these compounds are also resistant to an isoamylase $+\beta$-amylase one-pot reaction. The HPAEC chromatogram of potato IMMP clearly shows the effect of $\beta$-amylase on the unshielded $\alpha-(1 \rightarrow 4)$ linked glycosidic chains, since the pure $\alpha-(1 \rightarrow 4)$ peaks are completely removed with the introduction of $\beta$-amylase (Figure 3 (II) and Figure 3 (III)). The $\alpha-(1 \rightarrow 6)$ linked glycosidic 'lump' between $t_{e l}=20-35$ min remains unaffected by the combined isoamylase and $\beta$-amylase activity (Figure 3 (III)).

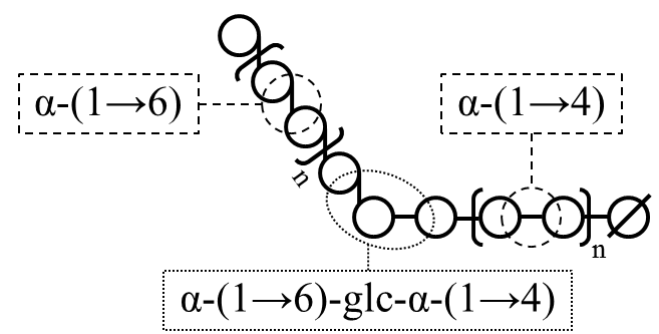

Figure 4. Depiction of a hybrid IMMP molecule with linear $\alpha-(1 \rightarrow 4)$ and $\alpha-(1 \rightarrow 6)$ linked glycosidic chains (dashed circles) connected by an $\alpha-(1 \rightarrow 6)$-glc- $\alpha-(1 \rightarrow 4)$ transition point (dotted oval) that can be specifically hydrolysed by the isopullulanase enzyme.

\subsubsection{CLD of $\alpha-(1 \rightarrow 4)$ linked glycosidic chains in hybrid molecules}

Hybrid molecules depicted in Figure 4 (Figure 2 (III) and Figure 3 (III)), can be investigated for their $\alpha-(1 \rightarrow 4)$ linked CLD by a two-step enzymatic treatment of the IMMP substrate. The first step consists of a one-pot reaction with dextranase, isoamylase and $\beta$-amylase that results in the partial hydrolysis of linear $\alpha-(1 \rightarrow 6)$ linked glycosidic material without affecting the shielded $\alpha-(1 \rightarrow 4)$ linked glycosidic material (Figure 2 (IV) and Figure 3 (IV)). After deactivation of the enzymes from the first step, the second step continues with an isopullulanase incubation (Figure $2(\mathrm{~V})$ and Figure 3 $(\mathrm{V})$ ), which reveals the CLD of the previously shielded $\alpha-(1 \rightarrow 4)$ linked glycosidic material. 
The first step (Figure 2 (IV) and Figure 3 (IV)), creates hybrid molecules with a majority of $\alpha-(1 \rightarrow 4)$ linked glycosidic linkages that are shielded by a few or only one $\alpha-(1 \rightarrow 6)$ linked glucoses. This dextranase is probably not able to fully hydrolyse linear $\alpha-(1 \rightarrow 6)$ linked glycosidic linkages near $\alpha-(1 \rightarrow 6)$-glc- $\alpha-(1 \rightarrow 4)$ transition points (Figure 4$)$, since we found no activity on pullulan (unpublished results). HPSEC chromatography shows a significant drop at $t_{e l}=11 \mathrm{~min}$ (Figure 2 (IV)), compared to the one-pot reaction with isoamylase and $\beta$-amylase (Figure 2 (III)), indicating that most $\alpha$ - $(1 \rightarrow 6)$ linked glycosidic material eluted at $\mathrm{t}_{\mathrm{el}}=11 \mathrm{~min}$. This is confirmed by HPAEC chromatography which shows the disappearance of the $\alpha-(1 \rightarrow 6)$ linked glycosidic 'lump' between $t_{e l}=$ 20-35 min and the appearance of compounds that elute closely but not synchronous to our $\alpha-(1 \rightarrow 4)$ linked glycosidic reference oligosaccharides (Figure 3 (IV), supplementary information 7.2).

After inactivation, isopullulanase was used to specifically hydrolyse the $\alpha-(1 \rightarrow 4)$ glycosidic linkage in the $\alpha-(1 \rightarrow 6)$-glc- $\alpha-(1 \rightarrow 4)$ transition point of the hybrid molecules (Figure 4). While HPSEC chromatography shows little change in $\mathrm{M}_{\mathrm{w}}$ after this treatment, HPAEC chromatography reveals that the compounds that appeared after dextranase treatment are now eluting synchronous to our $\alpha-(1 \rightarrow 4)$ linked glycosidic reference oligosaccharides (Figure $3(\mathrm{~V})$, supplementary information 7.2). This indicates that the isopullulanase treatment has indeed succeeded in revealing the CLD of the previously shielded $\alpha-(1 \rightarrow 4)$ linked glycosidic chains in the IMMP substrate (Figure 2 (I), black line).

\subsubsection{CLD of $\alpha-(1 \rightarrow 6)$ linked glycosidic chains in hybrid molecules}

The CLD of $\alpha-(1 \rightarrow 6)$ linked glycosidic chains in the hybrid molecules depicted in Figure 4 (Figure 2 (III) and Figure 3 (III)), can be investigated by an isoamylase, $\beta$ amylase and isopullulanase one-pot reaction of the IMMP substrate. The isopullulanase activity exposes the previously shielded $\alpha-(1 \rightarrow 4)$ linked non-reducing ends to $\beta$ amylase activity. Resulting in the hydrolysis of all linear $\alpha-(1 \rightarrow 4)$ linked glycosidic material into maltose and revealing the CLD of the linear $\alpha-(1 \rightarrow 6)$ linked glycosidic chains introduced by the GTFB- $\Delta \mathrm{N}$ enzyme.

Another interesting observation is that the combined isoamylase, $\beta$-amylase and isopullulanase treatment results in a fraction of $\alpha-(1 \rightarrow 6)$ linked glycosidic oligosaccharides that is exactly in the same range as the $\alpha-(1 \rightarrow 6)$ linked glycosidic oligosaccharides formed in the later stages of IMMP fermentation with faecal inoculum (Gu et al., 2018). This indicates that it is possible to artificially produce intermediate IMMP digestion products that can be used for future research on the digestion and possible health promoting effects of IMMPs. 


\subsection{Comparing enzymatic fingerprinting profiles of different IMMPs}

The strength of enzymatic fingerprinting is the ability to reveal different structural features in closely related polysaccharides. Since we are interested in studying the effect of the GTFB- $\Delta \mathrm{N}$ enzyme on different starch substrates, we use the differentiating ability of enzymatic fingerprinting to study different IMMPs. Potato IMMP and waxy potato IMMP were produced from amylose-rich potato starch and amylose-free potato starch respectively (van der Zaal et al., 2017), with the enzymatic fingerprinting approach outlined in Figures 3 and 5. Previously fractionated samples of a potato IMMP HMW and a potato IMMP LMW fraction (van der Zaal et al., 2017), were also fingerprinted (Figure 5), following the same consistent colour scheme as Figures 2 and 3. The fractionation of potato IMMP allows for a closer look at the effect of the presence of amylose on the enzymatic modification of amylopectin, since amylose is known to have a positive effect on the GTFB- $\Delta \mathrm{N}$ linear $\alpha-(1 \rightarrow 6)$ glycosidic linkage formation in IMMP HMW fractions (van der Zaal et al., 2017). A simplified overview of the results is summarized in Table 1 and will be discussed below.

Table 2. Simplified overview of the IMMP substructure analysis described in Figure 2 and the results depicted in Figures 3 and 5. The ${ }^{1} \mathrm{H}$ NMR total $\alpha-(1 \rightarrow 6)$ linked glycosidic linkage content $(\%)$ was obtained from van der Zaal et al. (2017). I, II, III, IV, V and VI refer to the different enzymatic treatments depicted in Figure 2.

\begin{tabular}{|l|c|c|c|c|c|c|}
\hline \multirow{2}{*}{ Sample } & \multicolumn{5}{|c|}{ IMMP substructure } \\
\cline { 4 - 8 } & $\begin{array}{c}\text { 1H NMR } \\
\text { total } \alpha-(1 \rightarrow 6) \\
\text { content (\%) }\end{array}$ & $\begin{array}{c}\text { HPAEC } \\
\alpha-(1 \rightarrow 6) \\
\text { lump' } \\
\text { lI, II, III, VI) }\end{array}$ & $\begin{array}{c}\text { Unshielded } \\
\alpha-(1 \rightarrow 4) \\
\text { linked } \\
\text { chains (II) }\end{array}$ & $\begin{array}{c}\text { Hybrid } \\
\text { molecules } \\
(\text { IV) }\end{array}$ & $\begin{array}{c}\text { Shielded } \\
\alpha-(1 \rightarrow 4) \\
\text { linked } \\
\text { chains (V) }\end{array}$ & $\begin{array}{c}\text { Linear } \\
\alpha-(1 \rightarrow 6) \\
\text { linked } \\
\text { chains (VI) }\end{array}$ \\
\hline Potato IMMP & 25 & ++ & ++ & +++ & +++ & +++ \\
\hline Waxy potato IMMP & 5 & - & +++ & + & + & + \\
\hline Potato IMMP HMW & 9 & - & ++ & +++ & +++ & ++ \\
\hline Potato IMMP LMW & 52 & +++ & + & +++ & +++ & +++ \\
\hline
\end{tabular}




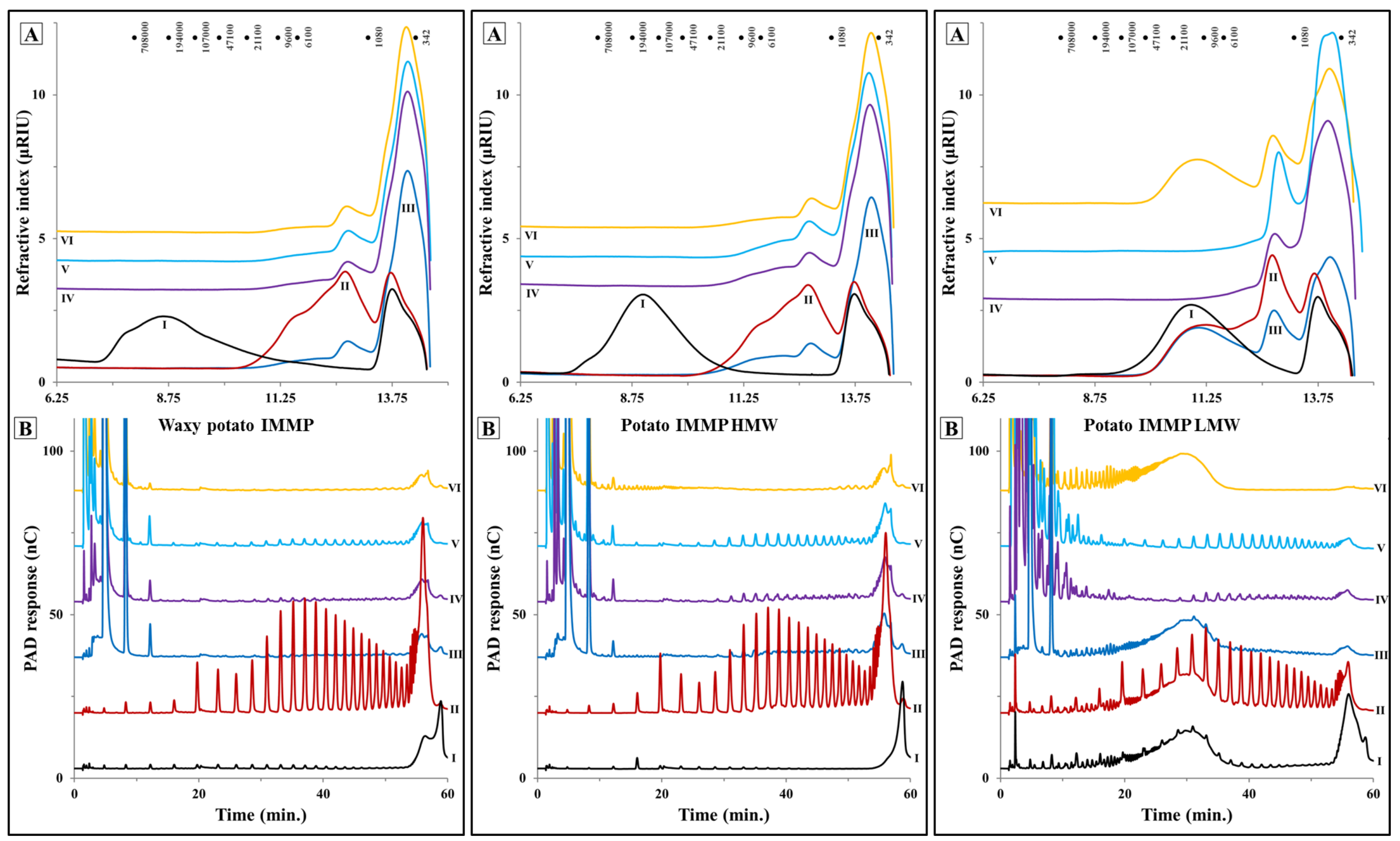

Figure 5. HPSEC (A) and HPAEC (B) profiles of waxy potato IMMP, potato IMMP HMW and potato IMMP LMW (I). Hydrolysed by isoamylase (II), one-pot incubation of isoamylase and $\beta$-amylase (III), one-pot incubation of isoamylase, $\beta$-amylase and dextranase (IV), one-pot incubation of isoamylase, $\beta$-amylase and dextranase followed by isopullulanase (V) and a one-pot incubation of isoamylase, $\beta$-amylase and isopullulanase (VI). 


\subsubsection{Potato IMMP}

HPSEC chromatography of potato IMMP after the combined action isoamylase and $\beta$ amylase shows a significant drop in Mw (Figure 3 (III)) compared to the isoamylase debranched IMMP (Figure 3 (II)). This indicates that unshielded $\alpha-(1 \rightarrow 4)$ linked glycosidic chains make up a large part of the original potato IMMP structure, which is in accordance with the $\sim 25 \% \alpha-(1 \rightarrow 6)$ glycosidic linkage content reported for potato IMMP (Table 1). HPSEC chromatography after the isoamylase, $\beta$-amylase and isopullulanase one-pot reaction (Figure $2(\mathrm{VI})$, revealed $\alpha-(1 \rightarrow 6)$ linked glycosidic chains up to $18.0 \mathrm{kDa}$ (Figure 3 (VI)). HPAEC analysis shows identifiable $\alpha-(1 \rightarrow 6)$ linked glycosidic chains up to DP 30 , the remainder of the $\alpha-(1 \rightarrow 6)$ glycosidic 'lump' is likely to contain even larger molecules (Figure 3 (VI)). Enzymatic fingerprinting of potato IMMP shows that only a part of the substrate acts as an acceptor for $\alpha-(1 \rightarrow 6)$ glycosidic chains, leaving quite some unshielded $\alpha-(1 \rightarrow 4)$ linked reducing ends available for hydrolysis with $\beta$-amylase. The minority of unshielded $\alpha-(1 \rightarrow 4)$ linked reducing ends that do function as an acceptor for the GTFB- $\Delta \mathrm{N}$ enzyme, show a substantial GTFB- $\Delta \mathrm{N}$ transferase activity, leading to the formation of long linear $\alpha$ $(1 \rightarrow 6)$ linked glycosidic chains in potato IMMP.

\subsubsection{Waxy potato IMMP}

HPSEC analysis shows that the combined action of isoamylase and $\beta$-amylase of waxy potato IMMP results in the smallest fraction of all the fingerprinted samples, indicating that waxy potato IMMP contains the highest amount of unshielded $\alpha-(1 \rightarrow 4)$ linked glycosidic chains and the lowest amount of shielded $\alpha-(1 \rightarrow 4)$ linked glycosidic chains of the four fingerprinted IMMPs (Figure 3 (III) and Figure 5 (III)). Which is not surprising, considering that the total $\alpha-(1 \rightarrow 6)$ content of waxy potato IMMP is $5 \%$ (Table 1). The biggest difference between waxy potato IMMP and potato IMMP is the lack of the $\alpha-(1 \rightarrow 6)$ glycosidic 'lump' between tel $=20-35$ min for waxy potato IMMP in the HPAEC chromatograms (Figure 3 (I, II, III, VI) and Figure 5 (I, II, III, VI)). Despite the lack of a large $\alpha-(1 \rightarrow 6)$ linked glycosidic population, there is still evidence for minor GTFB- $\Delta$ N transferase activity. The CLD of the $\alpha-(1 \rightarrow 4)$ linked glycosidic chains in hybrid molecules (Figure 5 (IV, V)), reveals trace amounts of shielded $\alpha$ $(1 \rightarrow 4)$ linked glycosidic chains. The CLD of the $\alpha-(1 \rightarrow 6)$ linked glycosidic chains in hybrid molecules (Figure $5(\mathrm{VI})$ ), reveals that the linear $\alpha-(1 \rightarrow 6)$ linked glycosidic chains shielding the $\alpha-(1 \rightarrow 4)$ linked glycosidic chains are only present in small amounts and have a relatively small DP compared to the other samples. Enzymatic fingerprinting of waxy potato IMMP shows that the absence of amylose results in lower GTFB- $\Delta \mathrm{N}$ 
transferase activity which, in turn, results in less shielding of $\alpha-(1 \rightarrow 4)$ linked reducing ends.

\subsubsection{Potato IMMP HMW}

Although the potato IMMP HMW fraction only contains at total $\alpha-(1 \rightarrow 6)$ glycosidic linkage content of $9 \%$, the $\alpha-(1 \rightarrow 6)$ linked glycosidic chains were found to be up to 13.5 $\mathrm{kDa}(\mathrm{HPSEC})$, with identifiable $\alpha-(1 \rightarrow 6)$ linked glycosidic chains of DP $=35$ (HPAEC) (Figure 5 (VI)). While the HPAEC chromatograms of potato IMMP HMW do not contain a clearly definable $\alpha-(1 \rightarrow 6)$ linked glycosidic 'lump' between $t_{\mathrm{el}}=20-35 \mathrm{~min}$, potato IMMP HMW does contain larger amounts of shielded $\alpha-(1 \rightarrow 4)$ linked glycosidic material (Figure $5(\mathrm{IV}, \mathrm{V})$ ) and bigger linear $\alpha-(1 \rightarrow 6)$ linked glycosidic chains shielding them (Figure 5 (VI)), compared to waxy potato IMMP (\$3.3.2). This shows that GTFB$\Delta \mathrm{N}$ modification of amylopectin on the absence or presence of amylose. Since the presence of amylose results in an IMMP HMW fraction that contains more shielded $\alpha$ $(1 \rightarrow 4)$ linked glycosidic chains with larger DP $\alpha-(1 \rightarrow 6)$ linked glycosidic chains shielding them.

\subsubsection{Potato IMMP LMW}

With a total $\alpha-(1 \rightarrow 6)$ glycosidic linkage content of $52 \%$, the potato IMMP LMW fraction clearly shows the impact of higher $\alpha-(1 \rightarrow 6)$ glycosidic linkage contents on HPSEC and HPAEC elution profiles. The fraction of $\alpha-(1 \rightarrow 6)$ linked glycosidic material elutes at $t_{\mathrm{el}}=11 \mathrm{~min}$ on HPSEC and between $\mathrm{t}_{\mathrm{el}}=20-35 \mathrm{~min}$ on HPAEC (Figure 5 (I, II, III, VI)). The potato IMMP LMW fraction is by far the most resistant fraction to the combined isoamylase and $\beta$-amylase treatment (Figure 5 (III)), indicating that the potato IMMP LMW fraction contains the lowest amount of unshielded $\alpha-(1 \rightarrow 4)$ linked glycosidic chains. The size of the $\alpha-(1 \rightarrow 6)$ linked glycosidic chains in the potato IMMP LMW fraction was found to be up to $20 \mathrm{kDa}$ (HPSEC) (Figure 5 (VI)), with an identifiable chain length up to DP = 55 in HPAEC (Figure 5 (VI)), the highest of all the investigated samples. This implies that a higher level of sample modification is correlated to the formation of larger individual $\alpha-(1 \rightarrow 6)$ linked glycosidic chains. 


\section{Conclusions}

The enzymatic fingerprinting method demonstrated in this study revealed detailed chain length distributions (CLD) of IMMP substructures and helped to provide new insights on GTFB- $\Delta$ N substrate interaction. We demonstrated that the enzymatic fingerprinting approach is able to differentiate between closely related substrates and able to pick up subtle differences. This makes enzymatic fingerprinting extremely qualified for the investigation of the reaction mechanism and preferences of glucanotransferase enzymes such as GTFB- $\Delta N$. Enzymatic fingerprinting helped to investigate the influence of amylose during IMMP synthesis. The presence of amylose resulted in the incorporation of linear $\alpha-(1 \rightarrow 6)$ linked glycosidic linkages in the amylopectin, while the absence of amylose only resulted in the incorporation of trace amounts. When linear $\alpha-(1 \rightarrow 6)$ linked glycosidic linkages were incorporated in the amylopectin it resulted in the elongation of single amylopectin chains rather than providing an even spread. Linear $\alpha$ $(1 \rightarrow 6)$ linked glycosidic chains introduced by the GTFB- $\Delta$ N enzyme were in some cases larger than $20 \mathrm{kDa}$, three times larger than previously determined (Leemhuis et al., 2014). It can be concluded that the enzymatic fingerprinting method has been able to detect and quantify detailed substructure compositions at a resolution that is not achievable with traditional techniques. This enzymatic fingerprinting method combined with ${ }^{1} \mathrm{H}$ NMR analysis could also function as an improved alternative for permethylation analysis. The knowledge obtained by the enzymatic fingerprinting of IMMPs will be used in forthcoming papers for a more in-depth investigation of GTFB reaction dynamics and for the directed enzymatic modification of $\alpha$-glucans.

\section{Acknowledgements}

This project (TKI-2013-B) is jointly funded by AVEBE and TKI as coordinated by the Carbohydrate Competence Center (CCC-ABC; www.cccresearch.nl). The authors thank Margaret Bosveld (Laboratory of Food Chemistry, Wageningen University \& Research) for her help and support with the analysis. 


\section{References}

Alting, A. C., van de Velde, Kanning, M. W., Burgering, M., Mulleners, L., Sein, A. \& Buwalda, P. (2009). Improved creaminess of low-fat yoghurt: The impact of amylomaltase-treated starch domains. Food Hydrocolloids, 23(3), 980-987. https://doi.org/10.1016/j.foodhyd.2008.07.011

An, H., Liang, H., Liu, Z., Yang, H., Liu, Q., \& Wang, H. (2011). Nano-structures of debranched potato starch obtained by isoamylolysis. Journal of Food Science, 76(1), 11-14. https://doi.org/10.1111/j.1750-3841.2010.01881.x

Bai, Y., Gangoiti, J., Dijkstra, B. W., Dijkhuizen, L., \& Pijning, T. (2016). Crystal Structure of 4,6- $\alpha$ Glucanotransferase Supports Diet-Driven Evolution of GH70 Enzymes from $\alpha$-Amylases in Oral Bacteria. Structure, 1-12. https://doi.org/10.1016/j.str.2016.11.023

Beeren, S. R., \& Hindsgaul, O. (2014). A fluorescence assay that detects long branches in the starch polysaccharide amylopectin. Chemical Communications (Cambridge, England), 50(13), 1530-2. https://doi.org/10.1039/c3cc48163h

Blazek, J., \& Gilbert, E. (2011). Application of small-angle X-ray and neutron scattering techniques to the characterisation of starch structure: A review. Carbohydrate Polymers, 85. https://doi.org/10.1016/j.carbpol.2011.02.041

Bourgoin, A., Zablackis, E., \& Poli, J. B. (2008). Characterization of $\alpha$-carrageenan solution behavior by field-flow fractionation and multiangle light scattering. Food Hydrocolloids, 22(8), 16071611. https://doi.org/10.1016/j.foodhyd.2007.11.001

Broxterman, S. E., Picouet, P., \& Schols, H. A. (2017). Acetylated pectins in raw and heat processed carrots. Carbohydrate Polymers, 177(August), 58-66.

https://doi.org/10.1016/j.carbpol.2017.08.118

Chen, Z., Schols, H. A., \& Voragen, A. G. J. (2004). Differently sized granules from acetylated potato and sweet potato starches differ in the acetyl substitution pattern of their amylose populations. Carbohydrate Polymers, 56(2), 219-226. https://doi.org/10.1016/j.carbpol.2004.02.004

Ciric, J., Woortman, A. J. J., \& Loos, K. (2014). Analysis of isoamylase debranched starches with size exclusion chromatography utilizing PFG columns. Carbohydrate Polymers, 112, 458-461. https://doi.org/10.1016/j.carbpol.2014.05.093

Dobruchowska, J. M., Gerwig, G. J., Kralj, S., Grijpstra, P., Leemhuis, H., Dijkhuizen, L., \& Kamerling, J. P. (2012). Structural characterization of linear isomalto-/malto-oligomer products synthesized by the novel GTFB 4,6- $\alpha$-glucanotransferase enzyme from Lactobacillus reuteri 121. Glycobiology, 22(4), 517-528. https://doi.org/10.1093/glycob/cwr167

Fontana, C., Li, S., Yang, Z., \& Widmalm, G. (2015). Structural studies of the exopolysaccharide from Lactobacillus plantarum C88 using NMR spectroscopy and the program CASPER. Carbohydrate Research, 402, 87-94. https://doi.org/10.1016/j.carres.2014.09.003

Gidley, M. J., \& Bulpin, P. V. (1989). Aggregation of Amylose in Aqueous Systems: The Effect of Chain Length on Phase Behavior and Aggregation Kinetics. Macromolecules, 22(13), 341-346. https://doi.org/10.1021/ma00191a062

Goesaert, H., Bijttebier, A., \& Delcour, J. A. (2010). Hydrolysis of amylopectin by amylolytic enzymes: level of inner chain attack as an important analytical differentiation criterion. Carbohydrate Research, 345(3), 397-401. https://doi.org/10.1016/j.carres.2009.11.011

Grewal, N., Faubion, J., Feng, G., Kaufman, R. C., Wilson, J. D., \& Shi, Y. C. (2015). Structure of waxy maize starch hydrolyzed by maltogenic $\alpha$-amylase in relation to its retrogradation. Journal 
of Agricultural and Food Chemistry, 63(16), 4196-4201. https://doi.org/10.1021/jf506215s

Grün, C. H., Sanders, P., van der Burg, M., Schuurbiers, E., van Adrichem, L., van Velzen, E. J. J., de Roo, N., Brunt, K., Westphal, Y. \& Schols, H. A. (2015). Strategy to identify and quantify polysaccharide gums in gelled food concentrates. Food Chemistry, 166, 42-49. https://doi.org/10.1016/j.foodchem.2014.05.129

Gu, F., Borewicz, K., Richter, B., van der Zaal, P. H., Smidt, H., Buwalda, P. L., \& Schols, H. A. (2018). In vitro fermentation behaviour of isomalto/malto-polysaccharides using human faecal inoculum indicates prebiotic potential. Molecular Nutrition \& Food Research. https://doi.org/10.1002/mnfr.201601015

Hizukuri, S., Kaneko, T., \& Takeda, Y. (1983). Measurement of the chain length of amylopectin and its relevance to the origin of crystalline polymorphism of starch granules. BBA - General Subjects, 760(1), 188-191. https://doi.org/10.1016/0304-4165(83)90142-3

Huijbrechts, A. A. M. L., Huang, J., Schols, H. A., Van Lagen, B., Visser, G. M., Boeriu, C. G., \& Sudhölter, E. J. R. (2007). 1-Allyloxy-2-hydroxy-propyl-starch: Synthesis and characterization. Journal of Polymer Science, Part A: Polymer Chemistry, 45(13), 2734-2744. https://doi.org/10.1002/pola.22029

Irague, R., Tarquis, L., Doublier, J. L., Moulis, C., \& Monsan, P. (2012). Structure and Property Engineering of $\alpha$-D-Glucans Synthesized by Dextransucrase Mutants. Biomacromolecules, 13, 187-195.

Kajiura, H., Takata, H., Kuriki, T., \& Kitamura, S. (2010). Structure and solution properties of enzymatically synthesized glycogen. Carbohydrate Research, 345(6), 817-824. https://doi.org/10.1016/j.carres.2010.01.013

Khalikova, E., Susi, P., \& Korpela, T. (2005). Microbial dextran-hydrolyzing enzymes: Fundamentals and applications. Microbiology and Molecular Biology Reviews, 69(2), 306-+. https://doi.org/Doi 10.1128/Jmrb.69.2.306-325.2005

Kim, B. K., Kim, H. I., Moon, T. W., \& Choi, S. J. (2014). Branch chain elongation by amylosucrase: Production of waxy corn starch with a slow digestion property. Food Chemistry, 152, 113-120. https://doi.org/10.1016/j.foodchem.2013.11.145

Lee, B.-H., Yan, L., Phillips, R. J., Reuhs, B. L., Jones, K., Rose, D. R., Nichols, B.L., QuezadaCalvillo, R., Yoo, S.H. \& Hamaker, B. R. (2013). Enzyme-Synthesized Highly Branched Maltodextrins Have Slow Glucose Generation at the Mucosal $\alpha$-Glucosidase Level and Are Slowly Digestible In Vivo. PLoS ONE, 8(4), e59745. https://doi.org/10.1371/journal.pone.0059745

Leemhuis, H., Dobruchowska, J. M., Ebbelaar, M., Faber, F., Buwalda, P. L., van der Maarel, M. J. E. C., Kamerling, J.P. \& Dijkhuizen, L. (2014). Isomalto/malto-polysaccharide, a novel soluble dietary fiber made via enzymatic conversion of starch. Journal of Agricultural and Food Chemistry, 62(49), 12034-12044. https://doi.org/10.1021/jf503970a

Lian, X., Kang, H., Sun, H., Liu, L., \& Li, L. (2015). Identification of the main retrogradation-related properties of rice starch. Journal of Agricultural and Food Chemistry, 63(5), 1562-1572. https://doi.org/10.1021/jf503203r

Ognyanov, M., Remoroza, C., Schols, H. A., Georgiev, Y., Kratchanova, M., \& Kratchanov, C. (2016). Isolation and structure elucidation of pectic polysaccharide from rose hip fruits (Rosa canina L.). Carbohydrate Polymers, 151, 803-811. https://doi.org/10.1016/j.carbpol.2016.06.031

Pérez, E., Gibert, O., Agnès, R.-S., Jiménez, Y., Sánchez, T., Giraldo, A., Pontoire, B., Guilois, S., Lahon, M.C., Reynes, M. \& Dufour, D. (2011). Physicochemical, functional, and 
macromolecular properties of waxy yam starches discovered from "Mapuey" (Dioscorea trifida) genotypes in the Venezuelan Amazon. Journal of Agricultural and Food Chemistry, 59(1), 263273. https://doi.org/10.1021/jf100418r

Pérez, S., \& Bertoft, E. (2010). The molecular structures of starch components and their contribution to the architecture of starch granules: A comprehensive review. Starch - Stärke, 62. https://doi.org/10.1002/star.201000013

Petersen, B. O., Motawie, M., Møller, B., Hindsgaul, O., \& Meier, S. (2015). NMR characterization of chemically synthesized branched $\alpha$-dextrin model compounds. Carbohydrate Research, 403, 149-156. https://doi.org/10.1016/j.carres.2014.05.011

Pettolino, F. A., Walsh, C., Fincher, G. B., \& Bacic, A. (2012). Determining the polysaccharide composition of plant cell walls. Nature Protocols, 7(9), 1590-1607. https://doi.org/10.1038/nprot.2012.081

Ray, S., Vigouroux, J., Quémener, B., Bonnin, E., \& Lahaye, M. (2014). Novel and diverse fine structures in LiCl-DMSO extracted apple hemicelluloses. Carbohydrate Polymers, 108(1), 4657. https://doi.org/10.1016/j.carbpol.2014.03.017

Remoroza, C., Broxterman, S., Gruppen, H., \& Schols, H. A. (2014). Two-step enzymatic fingerprinting of sugar beet pectin. Carbohydrate Polymers, 108(1), 338-347. https://doi.org/10.1016/j.carbpol.2014.02.052

Remoroza, C., Buchholt, H. C., Gruppen, H., \& Schols, H. A. (2014). Descriptive parameters for revealing substitution patterns of sugar beet pectins using pectolytic enzymes. Carbohydrate Polymers, 101(1), 1205-1215. https://doi.org/10.1016/j.carbpol.2013.10.034

Rolland-Sabaté, A., Sanchez, T., Buléon, A., Colonna, P., Ceballos, H., Zhao, S. S., Zhang, P. \& Dufour, D. (2013). Molecular and supra-molecular structure of waxy starches developed from cassava (Manihot esculenta Crantz). Carbohydrate Polymers, 92(2), 1451-1462. https://doi.org/10.1016/j.carbpol.2012.10.048

Schols, H. A., \& Voragen, A. G. J. (1996). Complex Pectins: Structure elucidation using enzymes. Progress in Biotechnology, 14(C), 3-19. https://doi.org/10.1016/S0921-0423(96)80242-5

Shen, X., Bertoft, E., Zhang, G., \& Hamaker, B. R. (2013). Iodine binding to explore the conformational state of internal chains of amylopectin. Carbohydrate Polymers, 98(1), 778-783. https://doi.org/10.1016/j.carbpol.2013.06.050

Siddiqui, N. N., Aman, A., Silipo, A., Qader, S. A. U., \& Molinaro, A. (2014). Structural analysis and characterization of dextran produced by wild and mutant strains of Leuconostoc mesenteroides. Carbohydrate Polymers, 99, 331-338. https://doi.org/10.1016/j.carbpol.2013.08.004

Song, G., \& Du, Q. (2012). Structure characterisation of a $\alpha \beta$-glucan polysaccharide from Auricularia polytricha. Natural Product Research, 26(21), 1963-1970. https://doi.org/10.1080/14786419.2011.633080

Sorndech, W., Sagnelli, D., Meier, S., Jansson, A. M., Lee, B., Hamaker, B. R., Rolland-Sabaté, A., Hebelstrup, K. H., Tongta, S. \& Blennow, A. (2016). Structure of branching enzyme- and amylomaltase modified starch produced from well-defined amylose to amylopectin substrates. Carbohydrate Polymers, 152, 51-61. https://doi.org/10.1016/j.carbpol.2016.06.097

Synytsya, A., \& Novak, M. (2014). Structural analysis of glucans. Annals of Translational Medicine, 2(2), 17. https://doi.org/10.3978/j.issn.2305-5839.2014.02.07

Tanackovic, V., Rydahl, M. G., Pedersen, H. L., Motawia, M. S., Shaik, S. S., Mikkelsen, M. D., Krunic, S. L., Fangel, J.U., Willats, W. G. T. \& Blennow, A. (2016). High throughput screening 
of starch structures using carbohydrate microarrays. Scientific Reports, 6, 30551.

https://doi.org/10.1038/srep30551

Tian, L., Gruppen, H., \& Schols, H. A. (2015). Characterization of (Glucurono)arabinoxylans from Oats Using Enzymatic Fingerprinting. Journal of Agricultural and Food Chemistry, 63(50), 10822-10830. https://doi.org/10.1021/acs.jafc.5b04419

van der Zaal, P. H., Schols, H. A., Bitter, J. H., \& Buwalda, P. L. (2017). Isomalto/maltopolysaccharide structure in relation to the structural properties of starch substrates. Carbohydrate Polymers, 185(November 2017), 179-186. https://doi.org/10.1016/j.carbpol.2017.11.072

Vilaplana, F., \& Gilbert, R. G. (2010). Characterization of branched polysaccharides using multipledetection size separation techniques. Journal of Separation Science, 33(22), 3537-3554. https://doi.org/10.1002/jssc.201000525

Voragen, A. G. J., Coenen, G. J., Verhoef, R. P., \& Schols, H. A. (2009). Pectin, a versatile polysaccharide present in plant cell walls. Structural Chemistry, 20(2), 263-275. https://doi.org/10.1007/s11224-009-9442-z

Wang, J., Zhao, X., Tian, Z., Yang, Y., \& Yang, Z. (2015). Characterization of an exopolysaccharide produced by Lactobacillus plantarum YW11 isolated from Tibet Kefir. Carbohydrate Polymers, 125, 16-25. https://doi.org/10.1016/j.carbpol.2015.03.003

Witt, T., \& Gilbert, R. G. (2014). Causal Relations between Structural Features of Amylopectin, a Semicrystalline Hyperbranched Polymer. Biomacromolecules, 15(7), 2501-2511. https://doi.org/10.1021/bm500353e 


\section{Supplementary information}

7.1 HPAEC elution speed comparison: $\alpha-(1 \rightarrow 4)$ versus $\alpha-(1 \rightarrow 6)$ linked glycosidic linkages

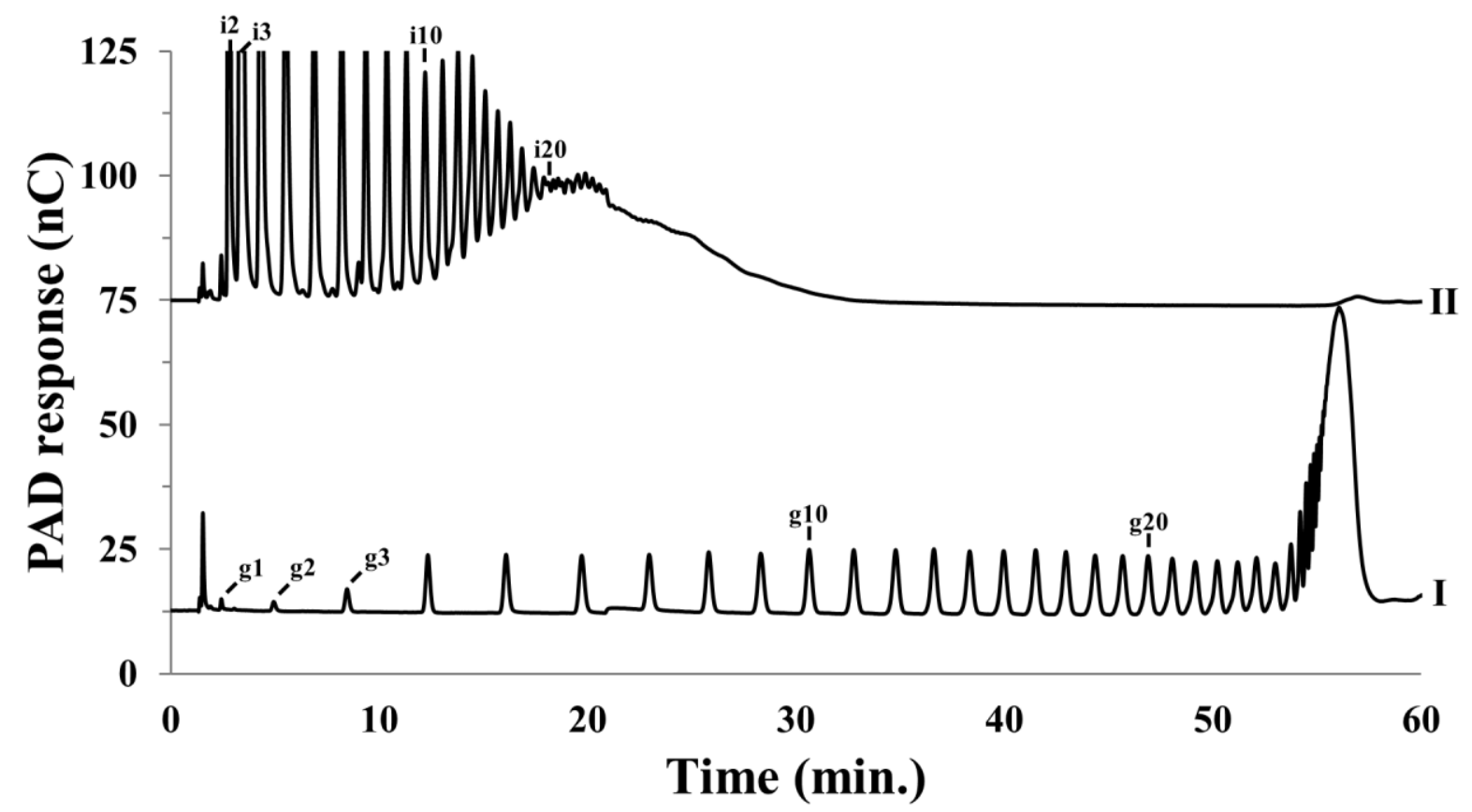

Isoamylase debranched amylomaltase treated potato starch (ATPS) $(\alpha-(1 \rightarrow 4)$ linked glycosidic reference oligosaccharides $(\mathbf{I}))$ and dextranase hydrolysed dextran $(\alpha-(1 \rightarrow 6)$ linked glycosidic reference oligosaccharides (II)). 


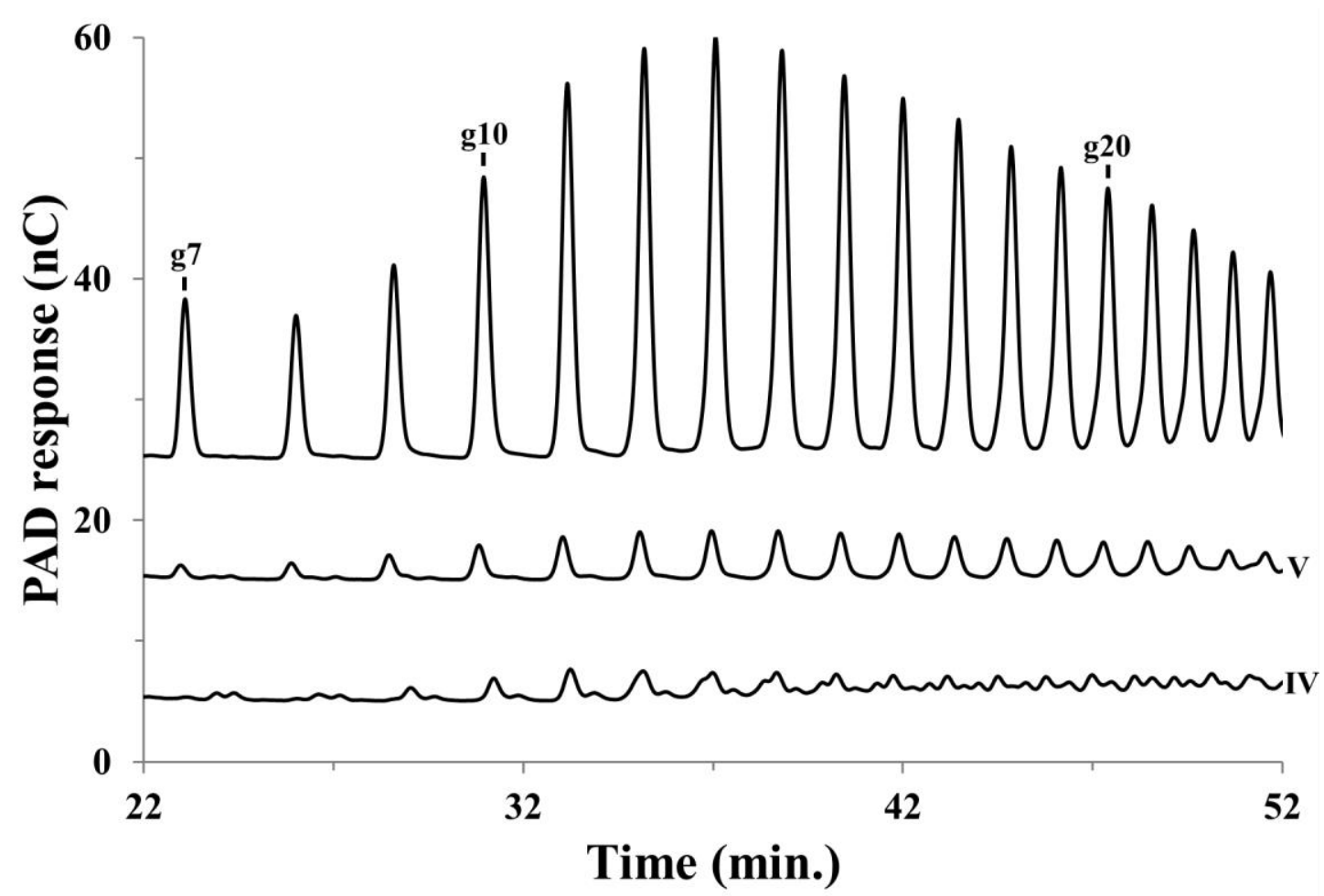

A comparison of the retention times of potato IMMP before (IV) and after (V) isopullulanase treatment. Isopullulanase treatment results in realignment with the $\alpha-(1 \rightarrow 4)$ linked glycosidic reference oligosaccharides, indicating that isopullulanase treatment results in the complete removal of shielding $\alpha-(1 \rightarrow 6)$ linked glycosidic material. IV refers to the one-pot incubation with isoamylase, $\beta$-amylase and dextranase (Figure 3 (IV, purple)) and $\mathbf{V}$ refers to the one-pot incubation with isoamylase, $\beta$-amylase and dextranase followed by isopullulanase (Figure 3 (V, light blue)). 



\section{Chapter 4}

\section{The influence of linear substrates on 4,6- $\alpha$-glucanotransferase reaction dynamics during isomalto/malto-polysaccharide synthesis}

Isomalto/malto-polysaccharides (IMMPs) are soluble dietary fibres produced by the incubation of $\alpha$-(1 $\rightarrow 4)$ linked $\alpha$-glucans with the 4,6- $\alpha$-glucanotransferase (GTFB) enzyme. This study investigates the reaction dynamics of the GTFB enzyme by using isoamylase treated starches as simplified linear substrates. GTFB modification was investigated over time and analysed with ${ }^{1} \mathrm{H}$ NMR, HPSEC, HPAEC combined with GOPOD and PAHBAH assays. In this paper we show that GTFB modification of linear substrates follows a substrate/acceptor model, in which $\alpha-(1 \rightarrow 4)$ linked glycosidic chains DP $\geq 6$ function as donor substrate, and $\alpha-(1 \rightarrow 4)$ linked glycosidic chains DP $<6$ function as acceptor. The presence of $\alpha-(1 \rightarrow 4)$ linked $\mathrm{DP}<6$ glycosidic material also resulted in higher GTFB transferase activity, while the absence resulted in higher GTFB hydrolytic activity. The information obtained in this study provides a better insight into GTFB reaction dynamics and will be useful for substrate selection for the directed modification of IMMPs in the future.

Based on: van der Zaal, P.H., Klostermann, C.E., Schols, H. A., Bitter J.H. \& Buwalda, P.L. (2018). The influence of linear substrates on 4,6- $\alpha$-glucanotransferase reaction dynamics during isomalto/malto-polysaccharide synthesis. Carbohydrate Polymers (to be submitted). 


\section{Introduction}

Isomalto/malto-polysaccharides (IMMPs) are $\alpha$-glucans produced by the enzymatic modification of starch with 4,6- $\alpha$-glucanotransferase (GTFB) (Leemhuis et al., 2014; van der Zaal et al., 2017). Modification of starch with the GTFB enzyme results in an increase of $\alpha-(1 \rightarrow 6)$ glycosidic linkages and a decrease of easily digestible $\alpha-(1 \rightarrow 4)$ glycosidic linkages. IMMPs are therefore considered a dietary fibre (Leemhuis et al., 2014) and previous research highlights their possible prebiotic potential ( $\mathrm{Gu}$ et al., 2018). In order to get a better understanding on GTFB modification, it is essential to understand how the GTFB enzyme interacts with its substrate.

In previous research, the activity of the GTFB enzyme was investigated on monodisperse model substrates, such as maltoheptaose (Dobruchowska et al., 2012). GTFB activity on maltoheptaose led to the formation of compounds smaller and larger than the initial substrate. This showed that the GTFB enzyme is able to polymerise $\alpha$ glucans by elongating linear $\alpha-(1 \rightarrow 6)$ linked glycosidic linkages at the expense of $\alpha$ $(1 \rightarrow 4)$ linked glycosidic linkages (Dobruchowska et al., 2012). This disproportionating effect is also commonly observed for other glucanotransferase enzymes, such as amylomaltase (4,4- $\alpha$-glucanotransferase) (van der Maarel et al., 2005). Experiments with monodisperse $\alpha$-glucans, however, are not easily transferable to technical applications, since the effect of varying substrate length on enzyme activity is not taken into account. This knowledge is needed since monodisperse $\alpha$-glucans are expensive and rare, normally one would like to use an easily obtainable substrate, such as starch.

Therefore, GTFB activity was also studied on starches, a more complex but widely available substrate (Bai et al., 2015; Leemhuis et al., 2014; van der Zaal et al., 2017). Starches generally have a high polydispersity index and vary in degree of branching and amylose content depending on the starch origin. Previous research on the GTFB modification of several starches shows the formation of large $\alpha-(1 \rightarrow 6)$ linked glycosidic chains, especially in the presence of amylose (van der Zaal et al., 2017; van der Zaal et al., 2018). The GTFB enzyme has a preference for linear $\alpha-(1 \rightarrow 4)$ linked substrate types, while $\alpha-(1 \rightarrow 4,6)$ linked branching points were limiting its performance (Leemhuis et al., 2014; van der Zaal et al., 2017). Although the use of starch is a lot more realistic in an industrial scenario, the complexity of the substrate made it hard to verify the underlying mechanism of the complex enzymatic disproportionation reaction.

In this study we 'simplify' our $\alpha$-glucan substrates with a debranching step. While a debranched $\alpha$-glucan substrate still has high polydispersity, its chain length distribution (CLD) can be controlled by selecting the type of starch that is debranched. For example, 
the CLD of amylomaltase treated starch is known to be broader, containing both smaller and larger material compared to debranched amylopectin (Ayudhaya, Pongsawasdi, Laohasongkram, \& Chaiwanichsiri, 2016). Therefore we selected debranched amylomaltase treated potato starch (ATPS) for its broad CLD and debranched waxy potato starch (WPS) for its narrow CLD. The effect of a broad and narrow CLD on GTFB activity was investigated over time and analysed with HPSEC, HPAEC, ${ }^{1} \mathrm{H}$ NMR and enzymatic fingerprinting. The use of simplified substrates in combination with several modes of detection allowed for an in-depth investigation on the limits of GTFB transferase activity. Which, in turn, will help to optimize the synthesis of future IMMPs. 


\section{Materials \& methods}

\subsection{Materials}

Waxy potato starch (WPS, Eliane 100) and amylomaltase treated potato starch (ATPS, Etenia 457 ) were provided by Avebe (Veendam, the Netherlands). Dextran ( 35 kDa), glucose, maltotriose and isomaltotriose were obtained from Sigma-Aldrich (St. Louis, MO, USA). Isomaltose was obtained from Megazyme (Bray, Wicklow, Ireland) and maltose was obtained from Fluka (Buchs, Switzerland). Isoamylase (EC 3.2.1.68) (Pseudomonas sp.), isopullulanase (EC 3.2.1.57) (Aspergillus niger) and $\beta$-amylase (EC 3.2.1.2) (Barley) were purchased from Megazyme (Bray, Wicklow, Ireland). Dextranase (EC 3.2.1.11) (Chaetomium erraticum) was obtained from Sigma-Aldrich (St. Louis, MO, USA).

\subsection{GTFB- $\triangle N$ production and activity}

4,6- $\alpha$-glucanotransferase- $\Delta \mathrm{N}$ (GTFB- $\Delta \mathrm{N})$ was produced and purified according to (van der Zaal et al., 2017). GTFB- $\Delta \mathrm{N}$ activity was measured with a GOPOD assay (Megazyme, Bray, Wicklow, Ireland) and was comparable to previous research (Bai et al., 2015; van der Zaal et al., 2017).

\subsection{IMMP synthesis from linear substrates}

WPS or ATPS was suspended at $2.5 \mathrm{mg} / \mathrm{mL}$ in $20 \mathrm{mM}$ acetate buffer, $\mathrm{pH}=5.0$ containing $5 \mathrm{mM} \mathrm{CaCl}_{2}$. The suspension was autoclaved at $121{ }^{\circ} \mathrm{C}$ for $15 \mathrm{~min}$. and cooled to $40{ }^{\circ} \mathrm{C}$. The reaction mixture was debranched by adding isoamylase $(0.16 \mathrm{U} / \mathrm{mg}$ substrate $)$ and incubating in a Kuhnershaker Climo-shaker IFF1-X (Kuhner, Bisfelden, Switzerland) at $40{ }^{\circ} \mathrm{C}$ and $100 \mathrm{rpm}$ for $17 \mathrm{~h}$. The isoamylase was inactivated in a water bath at $95^{\circ} \mathrm{C}$ for 15 minutes and the samples were subsequently cooled to $37^{\circ} \mathrm{C}$. IMMP synthesis was carried out by adding $0.3 \mathrm{mg}$ GTFB- $\Delta \mathrm{N} / \mathrm{g}$ substrate and incubating at $37^{\circ} \mathrm{C}$ for $0,7,24$, 48 and $70 \mathrm{~h}$. After reaction, GTFB- $\Delta \mathrm{N}$ was inactivated in a water bath at $95^{\circ} \mathrm{C}$ for 15 minutes. Samples were stored at room temperature to avoid crystallisation and analysed within 3 days after preparation.

\subsection{Enzymatic fingerprinting assay}

IMMPs synthesized from debranched ATPS were further analysed with an enzymatic fingerprinting method adapted from van der Zaal et al. (2018). The sample solutions $(2.5 \mathrm{mg} / \mathrm{mL})$ were incubated with different hydrolytic enzymes in a $20 \mathrm{mM}$ acetate buffer, $\mathrm{pH}=5.0$ containing $5 \mathrm{mM} \mathrm{CaCl} 2 . \beta$-amylase and isopullulanase were added at 
concentrations of $0.16 \mathrm{U} / \mathrm{mg}$ substrate and dextranase was added at a concentration of $0.052 \mathrm{U} / \mathrm{mg}$ substrate. The following incubations were performed: $\beta$-amylase, isopullulanase, dextranase individually, and two one-pot incubations of $\beta$-amylase with isopullulanase and $\beta$-amylase with dextranase. All incubations were performed in a Kuhnershaker at $39^{\circ} \mathrm{C}$ and $100 \mathrm{rpm}$ for $4 \mathrm{~h}$. After reaction, the enzymes were inactivated in a water bath at $95^{\circ} \mathrm{C}$ for $15 \mathrm{~min} . \alpha-(1 \rightarrow 6)$ and $\alpha-(1 \rightarrow 4)$ linked reference samples were prepared in similar fashion by incubating dextran $(2.5 \mathrm{mg} / \mathrm{mL})$ with dextranase $(0.052 \mathrm{U} / \mathrm{mg})$ and by incubating ATPS $(2.5 \mathrm{mg} / \mathrm{mL})$ with isoamylase $(0.16 \mathrm{U} / \mathrm{mg})$ respectively (supplementary information 7.1).

\section{$2.5^{1} H$ NMR Spectroscopy}

The total $\alpha-(1 \rightarrow 6)$ content was measured with ${ }^{1} \mathrm{H}$ NMR spectroscopy adapted from (van der Zaal et al., 2017). Freeze-dried IMMP was exchanged once with $\mathrm{D}_{2} \mathrm{O}$ by lyophilisation and dissolved in $\mathrm{D}_{2} \mathrm{O}$ (99.9 atom \% D, Sigma-Aldrich, St. Louis, MO, USA) at a concentration of $10 \mathrm{mg} / \mathrm{ml}$. Samples were shaken and heated up to $340 \mathrm{~K}$ in a Thermomixer (Eppendorf, Hamburg, Germany) to ensure maximal solubility during the NMR measurement. 1D ${ }^{1} \mathrm{H}$ NMR spectra were recorded at $340 \mathrm{~K}$ on a Bruker Avance 600 spectrometer equipped with a cryo-probe (Billerica, MA, USA) located at the Wageningen NMR Centre. Structures were characterized using the ${ }^{1} \mathrm{H}$ NMR structuralreporter-group concept for $\alpha$-D-glucans (Dobruchowska et al., 2012; van Leeuwen et al., 2008).

\subsection{Determination of molecular weight distribution by HPSEC-RI}

Sample solutions $(2.5 \mathrm{mg} / \mathrm{mL})$ were centrifuged at $7317 \mathrm{x} \mathrm{g}$ and $20{ }^{\circ} \mathrm{C}$ for $10 \mathrm{~min}$. and the supernatant was used for HPSEC analysis. An Ultimate 3000 HPLC (Dionex, Sunnyvale, USA) was used with a column set which consisted of three in series connected TosoHaas (Tokyo, Japan) TSK-Gel columns (4000PWXL-3000PWXL2500PWXL), (6 x $150 \mathrm{~mm}$ ), with a guard column and a Shodex type RI-101 refractive index detector (Showa Denko, K.K., Kawasaki, Japan). With 0.2 M NaNO3 as eluent and a flow of $0.6 \mathrm{~mL} / \mathrm{min}$ at $55^{\circ} \mathrm{C}$. A volume of $10 \mu \mathrm{L}$ sample solution was injected onto the column. A pullulan standard series (180 - 780000 Da) (Fluka) was used for calibration. The buffer and all enzymes were run separately as controls. Data analysis was performed with ChromeleonTM 7.1 software from Thermo Fisher Scientific (Waltham, Massachusetts, USA). 


\subsection{Determination of mono- and oligomers by HPAEC-PAD}

Sample solutions were diluted to $0.5 \mathrm{mg} / \mathrm{mL}$ with Millipore water and centrifuged at $7317 \mathrm{x} \mathrm{g}$ and $20^{\circ} \mathrm{C}$ for $10 \mathrm{~min}$, the supernatant was used for HPAEC analysis. The analysis was performed on an ICS5000 High Performance Anion Exchange Chromatography system with Pulsed Amperometric Detection (HPAEC-PAD) (Dionex Corporation, Sunnyvale, USA) equipped with a CarboPac PA-1 column ( 2 x $250 \mathrm{~mm})$ and a CarboPac PA-1 guard column $(2 \times 25 \mathrm{~mm})$. The two mobile phases were (A) 0.1 $\mathrm{M} \mathrm{NaOH}$ and (B) $1 \mathrm{M} \mathrm{NaOAc}$ in $0.1 \mathrm{M} \mathrm{NaOH}$ and the flow rate was set to $0.3 \mathrm{~mL} / \mathrm{min}$. The following gradient was used: 0-50 min 5-40\% B, 50-65 min 40-100\% B, 65-70 min $100 \%$ B ending with a $70-85$ min re-equilibration at $5 \% \mathrm{~B}$. A volume of $10 \mu \mathrm{L}$ sample solution was injected onto the column. Glucose, maltose, maltotriose, isomaltose and isomaltotriose $(10-100 \mu \mathrm{g} / \mathrm{mL})$ were run as standards. Data analysis was performed with ChromeleonTM 7.1 software Thermo Fisher Scientific (Waltham, Massachusetts, USA).

\subsection{Free glucose determination}

Free glucose was measured with the GOPOD assay (Megazyme, Bray, Wicklow, Ireland), the preparation of the GOPOD assay was performed according to the supplier's manual. The deactivated samples $(50 \mu \mathrm{L})$ were mixed with $1.5 \mathrm{~mL}$ of GOPOD reagents and incubated in a Thermomixer (Eppendorf, Hamburg, Germany) at $40{ }^{\circ} \mathrm{C}$ and $450 \mathrm{rpm}$ for 20 minutes. The absorbance at $510 \mathrm{~nm}$ was measured with a DU $720 \mathrm{UV} / \mathrm{vis}$ spectrophotometer (Beckman Coulter, Brea, CA, USA).

\subsection{Reducing ends determination}

The amount of reducing ends was measured at different time points during IMMP synthesis by using a 4-hydroxybenzoic acid hydrazide (PAHBAH) reagents. The deactivated samples (sugars) were diluted 3 or 4 times to fit the $0-1 \mathrm{mg} / \mathrm{mL}$ glucose calibration curve. A solution of $5 \%$ PAHBAH in $0.5 \mathrm{M} \mathrm{HCl}$ was mixed with 4 parts of $0.5 \mathrm{M} \mathrm{NaOH}$. The activated PAHBAH reagents $(200 \mu \mathrm{L})$ was added to $10 \mu \mathrm{L}$ sample in a 96-well plate. The 96-well plate was covered and incubated in a Thermomixer (Thermomixer, Hamburg, Germany) at $70{ }^{\circ} \mathrm{C}$ and $600 \mathrm{rpm}$ for 35 minutes. After cooling to room temperature, the absorbance was measured at $405 \mathrm{~nm}$ with a Tecan Infinite F500 microplate reader (Männedorf, Switzerland). 


\section{Result \& Discussion}

\subsection{Substrate chain length distribution}

Isoamylase was used to debranch amylomaltase treated potato starch (ATPS) and waxy potato starch (WPS). The chain length distribution (CLD) of debranched ATPS (dATPS) and debranched WPS (dWPS) is displayed in Figure 1. HPAEC peaks eluting before $t_{\mathrm{el}}=15$ min. reveal the presence of smaller DP $<6$ material in dATPS (Figure 1). HPSEC profiles show that dATPS contains more high molecular weight material compared to dWPS, see below. The presence of smaller and larger compounds in dATPS is caused by the disproportionating effect of amylomaltase treatment and results in a broader CLD (Ayudhaya et al., 2016). The HPAEC profile of dWPS contains fewer peaks compared to the HPAEC profile of dATPS and is thus distributed in a more narrow range, albeit still polydisperse (Figure 1). Both substrates consist solely of $\alpha$ $(1 \rightarrow 4)$ linked glycosidic material and vary only in their respective CLDs. Since $\alpha-(1 \rightarrow 4)$ linked glycosidic chains are considered to be the best known substrate for the GTFB$\Delta \mathrm{N}$ enzyme (Bai et al., 2015; Leemhuis et al., 2014; van der Zaal et al., 2017), both substrates should be perfectly suited for GTFB- $\Delta$ N modification.

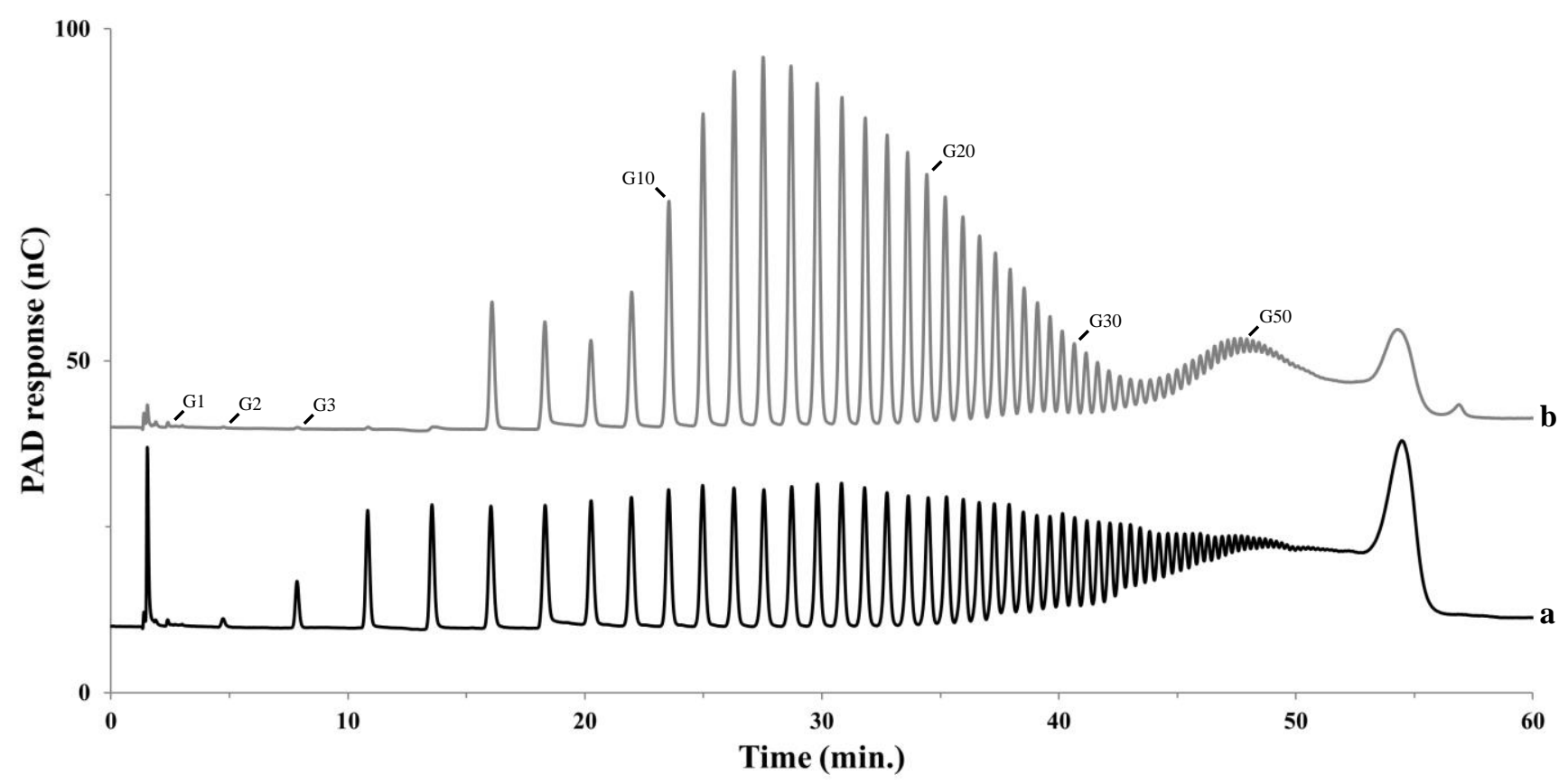

Figure 1. HPAEC profile of debranched amylomaltase treated potato starch (dATPS) (a) and debranched waxy potato starch (dWPS) (b). The elution times of the HPAEC standards; glucose, maltose, maltotriose are indicated as G1, G2, G3 respectively. 


\subsection{Hydrolytic and transferase activity of $G T F B-\Delta N$}

dATPS and dWPS were treated with the GTFB- $\Delta$ N enzyme and incubated for $0,7,24$, 48 or 70 hours. Previous research has shown that the GTFB enzyme displays both hydrolytic and transferase activity, with the hydrolytic activity splitting $\alpha-(1 \rightarrow 4)$ glycosidic linkages and the transferase activity forming $\alpha-(1 \rightarrow 6)$ linked glycosidic linkages from $\alpha-(1 \rightarrow 4)$ linked glycosidic linkages (Bai et al., 2015). GTFB- $\Delta \mathrm{N}$ hydrolytic activity was monitored by tracking the percentage of reducing ends and free glucose and GTFB- $\Delta$ N transferase activity was monitored by tracking the percentage of $\alpha-(1 \rightarrow 6)$ linked glycosidic linkages (Figure 2). Figure 2 shows that GTFB- $\Delta N$ transferase activity lags behind initial GTFB- $\Delta \mathrm{N}$ hydrolytic activity, since the amount of reducing ends and the amount of free glucose increase faster than the total amount of $\alpha-(1 \rightarrow 6)$ glycosidic linkages. The lag phase for transferase activity is shorter for dATPS and dATPS also scores consistently higher in total $\alpha-(1 \rightarrow 6)$ glycosidic linkage content, amount of free glucose and the amount of reducing ends compared to dWPS (Figure 2). The percentage $\alpha-(1 \rightarrow 6)$ linked glycosidic linkages after 70 hours of GTFB- $\Delta \mathrm{N}$ incubation is $69 \%$ for dATPS and $63 \%$ for dWPS. These initial measurements indicate that higher rates of GTFB- $\Delta \mathrm{N}$ transferase activity is preceded by initial hydrolytic activity, even in purely linear samples.
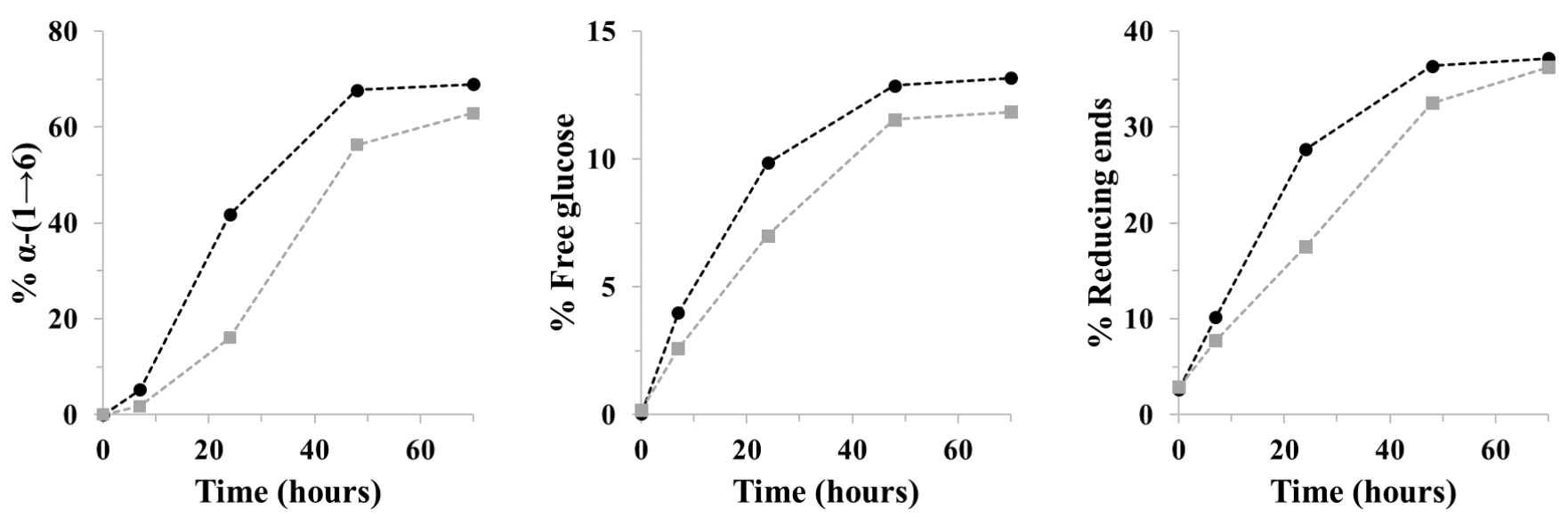

Figure 2. Incubation of debranched amylomaltase treated potato starch (dATPS) (•) and debranched waxy potato starch (dWPS) ( $\square$ ) with the GTFB- $\Delta$ N enzyme, measured at $0,7,24,48$ and 70 hours. The total $\alpha-(1 \rightarrow 6)$ linked glycosidic linkage content, free glucose and reducing ends are expressed as percentage of the total amount of free and bound glucose present in the sample. 


\subsection{The effect of GTFB- $\Delta N$ activity on molecular weight}

GTFB- $\triangle$ N modification of dATPS and dWPS was investigated in further detail with HPSEC and HPAEC (Figure 3). HPSEC profiles show that both dATPS and dWPS gain wider CLDs after GTFB- $\triangle$ N modification, with CLDs that contain larger and smaller populations than the initial substrate (Figure 3 (A)). Although we observe high polydispersity in all samples, the largest population of dATPS shows an average size increase from $5.8 \mathrm{kDa}$ to $9.5 \mathrm{kDa}$ and the largest population of dWPS shows an average size increase from $3.2 \mathrm{kDa}$ to $6.8 \mathrm{kDa}$ after 70 hours of incubation with GTFB- $\Delta \mathrm{N}$. This indicates that GTFB- $\Delta \mathrm{N}$ is still able to polymerise $\alpha$-glucans (Figure 3 (A)), despite the considerable increase in the amount of free glucose and reducing ends (Figure 2).

HPAEC profiles of both samples show a transition from a majority $\alpha-(1 \rightarrow 4)$ glycosidic linked substrate to a majority $\alpha-(1 \rightarrow 6)$ glycosidic linked product during the 70 hour incubation (Figure $3(\mathrm{~B})$ ). This transition is clearly visible in the HPAEC profiles (Figure $3(\mathrm{~B})$ ), since $\alpha-(1 \rightarrow 6)$ linked glycosidic material elutes faster than $\alpha-(1 \rightarrow 4)$ linked glycosidic material (van der Zaal et al. (2018), supplementary information 7.1). We can follow the transition of $\alpha-(1 \rightarrow 4)$ linked glycosidic substrate into $\alpha-(1 \rightarrow 6)$ linked glycosidic product by following the disappearance of the recognizable $\alpha-(1 \rightarrow 4)$ peaks (G2, G3, G10 and G20, Figure 3 (B)), or by following the appearance of the 'hump' of majority $\alpha-(1 \rightarrow 6)$ glycosidic linked material that is eluting between 20 and 25 minutes.

After 48 hours for dATPS and 70 hours for dWPS, only five $\alpha-(1 \rightarrow 4)$ linked peaks are left in the reaction mixture (Figure 3 (B)). Most of these leftover peaks are not naturally present in the substrate, they are the by-product of GTFB hydrolytic and transferase activity. These leftover $\alpha-(1 \rightarrow 4)$ peaks $(\mathrm{DP}<6)$ eluting before $t_{\mathrm{el}}=15 \mathrm{~min}$. (Figure 3 (B): dATPS $(t=48$ and 70), dWPS $(t=70)$ ), clearly indicate the limits of what the GTFB- $\Delta \mathrm{N}$ enzyme is still able to recognize and use as a donor substrate. This apparent minimum substrate length $(\mathrm{DP}=6)$ coincides with the minimum length that is needed to form a single $\alpha-(1 \rightarrow 4)$ glycosidic helix (Pérez \& Bertoft, 2010). The $\alpha-(1 \rightarrow 4)$ linked glycosidic compounds with a $\mathrm{DP}<6$ are therefore probably not recognized as donor substrate by the carbohydrate binding module (CBM) of the GTFB- $\Delta$ N enzyme. After 70 hours of incubation, there are some peaks that deviate from the $\alpha-(1 \rightarrow 6)$ glycosidic linked and $\alpha-(1 \rightarrow 4)$ glycosidic linked reference samples (Figure 3 (B), supplementary information 7.1). These are most likely hybrid molecules consisting of both an $\alpha-(1 \rightarrow 6)$ glycosidic linked segment and an $\alpha-(1 \rightarrow 4)$ glycosidic linked segment (van der Zaal et al., 2018). 

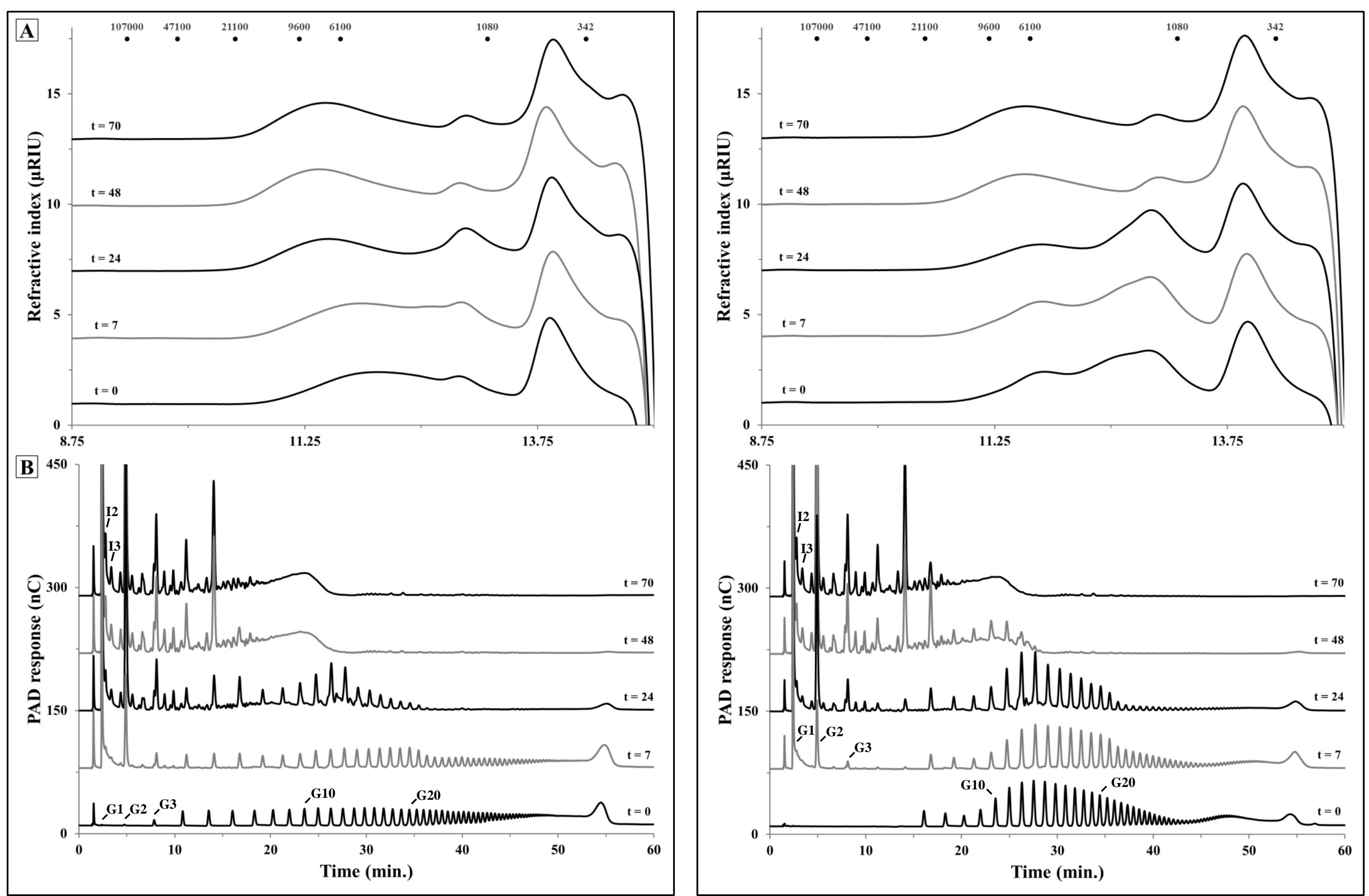

Figure 3. HPSEC (A) and HPAEC (B) profiles of isoamylase debranched amylomaltase treated potato starch (dATPS, left) and isoamylase debranched waxy potato starch (dWPS, right), incubated with GTFB- $\Delta \mathrm{N}$ for $0,7,24,48$ and 70 hours. HPSEC pullulan standards are indicated by black dots $(\bullet)$ and are given in Dalton (Da). The elution times of the HPAEC standards; glucose, maltose, maltotriose, isomaltose and isomaltotriose are indicated as G1, G2, G3, I2 and I3 respectively. 
The increase of glucose (G1), maltose (G2) and maltotriose (G3) after 7 hours of reaction time, indicates a mostly hydrolytic GTFB- $\Delta \mathrm{N}$ activity at the start of the reaction (Figure $3(B)$ ). The increase in linear $\alpha-(1 \rightarrow 4)$ linked glycosidic oligosaccharides, such as maltose and maltotriose, proves that the GTFB- $\Delta$ N enzyme is indeed capable of endoactivity as demonstrated in previous research (Bai et al., 2016), since exo-activity alone would only yield an increase in glucose.

The change in chain length distribution during GTFB- $\Delta \mathrm{N}$ modification shows that a majority $\alpha-(1 \rightarrow 4)$ linked glycosidic chains are converted into $\alpha-(1 \rightarrow 6)$ linked glycosidic chains (Figure $3(\mathrm{~B})$ ). The composition at the end of the reaction reveals that only $\alpha-$ $(1 \rightarrow 4)$ linked glycosidic chains $\mathrm{DP} \geq 6$ are a suitable donor substrate for GTFB- $\Delta \mathrm{N}$ transferase activity. The lag between GTFB- $\Delta \mathrm{N}$ transferase activity and GTFB- $\Delta \mathrm{N}$ hydrolytic activity indicates that the $\alpha-(1 \rightarrow 4)$ linked glycosidic moieties $(\mathrm{DP}<6)$ created by GTFB- $\Delta \mathrm{N}$ hydrolysis could potentially function as acceptors for GTFB transferase activity (Figure 2 and 3 ). These acceptors, in turn, might be able to increase the speed of the GTFB- $\Delta \mathrm{N}$ transferase activity, since previous research has shown that GTFB amylose degradation increases after the addition of low DP glycosidic acceptors (Bai et al., 2015).

\subsection{Enzymatic fingerprinting}

The incubation of dATPS with the GTFB- $\Delta \mathrm{N}$ enzyme was monitored by using an enzymatic fingerprinting method adapted from van der Zaal et al. (2018). Enzymatic fingerprinting was used in order to confirm the presence of hybrid molecules and to get more information on size of the linear $\alpha-(1 \rightarrow 6)$ linked glycosidic chains produced by the GTFB- $\triangle \mathrm{N}$ enzyme. Analysis of the reaction products also provided more information about the preferred substrate and acceptors of the GTFB- $\Delta$ N enzyme.

dATPS was incubated for 70 hours with the GTFB- $\triangle \mathrm{N}$ enzyme (dATPS $70 \mathrm{~h}$ ) and subsequently fingerprinted, HPSEC profiles of dATPS $70 \mathrm{~h}$ and the corresponding fingerprinted samples are displayed in Figure 4. All of the $\alpha-(1 \rightarrow 4)$ linked glycosidic material of the dATPS $70 \mathrm{~h}$ sample was removed by a one-pot incubation with isopullulanase and $\beta$-amylase (Figure $4,-$ ). The slight decrease in size indicates that most $\alpha$ - $(1 \rightarrow 6)$ linked glycosidic material contains a small amount of $\alpha-(1 \rightarrow 4)$ linked glycosidic linkages, but that most of the high molecular weight (HMW) fraction in dATPS $70 \mathrm{~h}$ consists of $\alpha-(1 \rightarrow 6)$ linked glycosidic material. When comparing the HPSEC profiles with the pullulan standards we can observe that GTFB- $\Delta \mathrm{N}$ is able to synthesize linear $\alpha-(1 \rightarrow 6)$ linked glycosidic chains up to $21 \mathrm{kDa}$, which is slightly 
higher than previously reported (van der Zaal et al., 2018). The one-pot incubation with dextranase and $\beta$-amylase hydrolyses both the $\alpha-(1 \rightarrow 6)$ linked glycosidic material and the $\alpha-(1 \rightarrow 4)$ linked glycosidic material with an available reducing end (Figure $4,-$ ). The large decrease in the HMW fraction also confirms that the HMW fraction of dATPS $70 \mathrm{~h}$ indeed consists of mostly of $\alpha-(1 \rightarrow 6)$ linked glycosidic material.

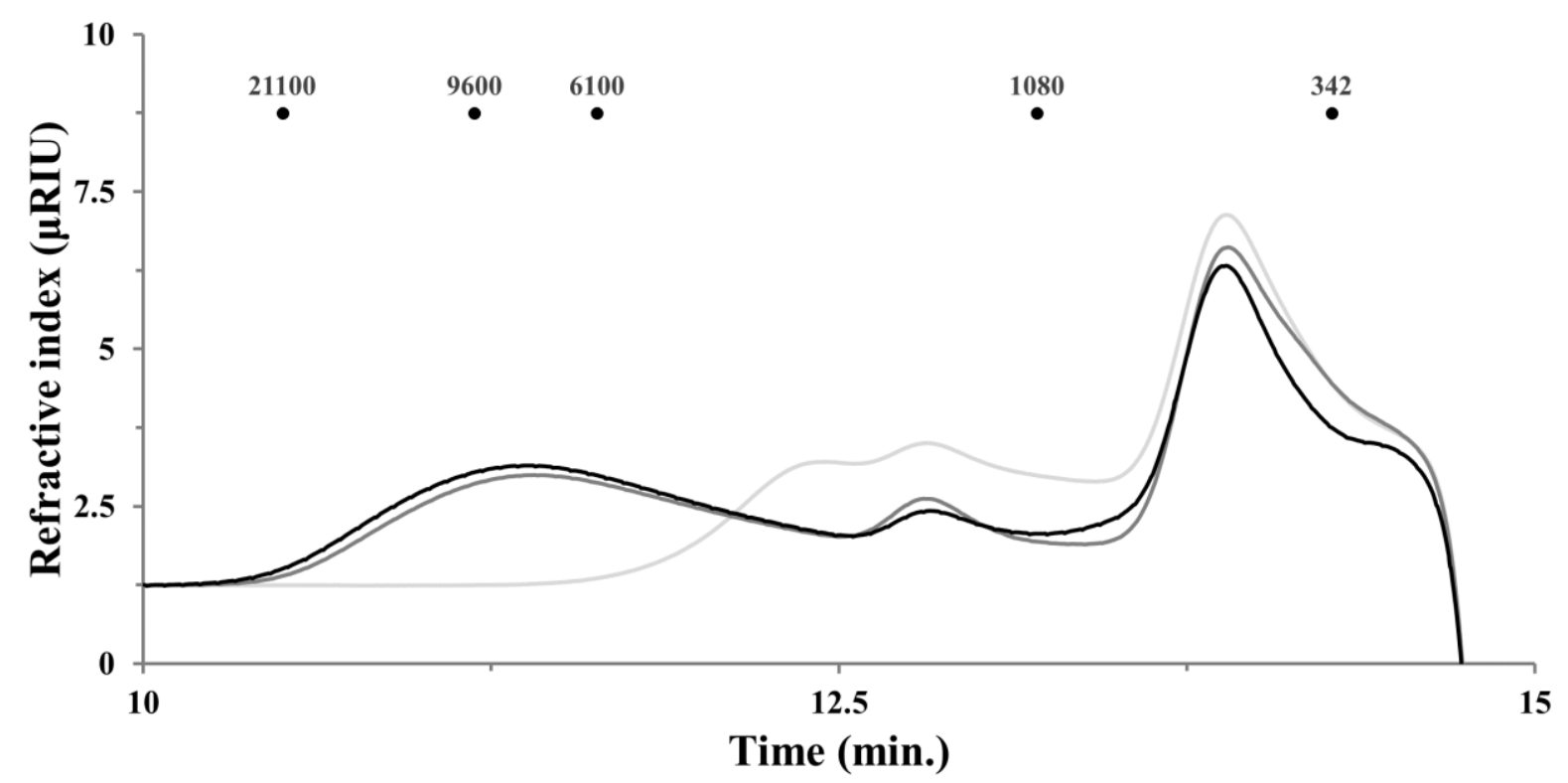

Figure 4. HPSEC profile of debranched amylomaltase treated potato starch after 70 hours of incubation with the GTFB- $\Delta$ N enzyme (dATPS $70 \mathrm{~h},-$ ), fingerprinted by a one-pot incubation with isopullulanase and $\beta$-amylase $(-)$ or by a one-pot incubation with dextranase and $\beta$-amylase $(-)$.

Different acceptors of GTFB- $\Delta \mathrm{N}$ transferase activity were identified by incubating dATPS $70 \mathrm{~h}$ with isopullulanase and comparing it with the blank (Figure 5). HPAEC chromatography shows a clear increase for maltose, maltotriose and maltotetraose after isopullulanase activity (Figure 5). As depicted in Figure 6, the existence of; maltose, maltotriose, maltotetraose, maltopentaose and maltohexaose acceptors can be proven by the respective increase in glucose (g1), maltose (g2), maltotriose (g3), maltotetraose (g4) and maltopentaose (g5) after isopullulanase incubation. The isopullulanase incubation thus indicates that maltotriose, maltotetraose and maltopentaose are relatively good acceptors for GTFB- $\Delta \mathrm{N}$ transferase activity. The large glucose peak was not reliable for quantification, but measurement of free glucose with the GOPOD assay showed an increase after isopullulanase incubation, proving that maltose is also used as an acceptor by the GTFB- $\Delta \mathrm{N}$ enzyme. The presence of panose in the dATPS $70 \mathrm{~h}$ sample also confirms the existence of maltose as acceptor for GTFB- $\Delta \mathrm{N}$ transferase activity (Figure 
5 and 6). The presence of isomaltose in the dATPS $70 \mathrm{~h}$ sample also proves the existence of glucose as acceptor (Figure 5 and 6). The increase in maltopentaose after isopullulanase incubation was minimal, meaning that we cannot really confirm or deny the existence of maltohexaose acceptors for GTFB- $\Delta$ N transferase activity (Figure 6), although maltohexaose was identified as an acceptor for GTFB- $\Delta$ N transferase activity in previous research (Dobruchowska et al., 2012). Their relative increase after isopullulanase incubation, however, indicates that maltotriose, maltotetraose and maltopentaose can be considered as the best acceptors for GTFB- $\Delta \mathrm{N}$ transferase activity. The combination of enzymatic fingerprinting and chromatography made this the first study that was able to extensively characterize and prove the existence of high DP IMMP structures depicted in Figure 6.

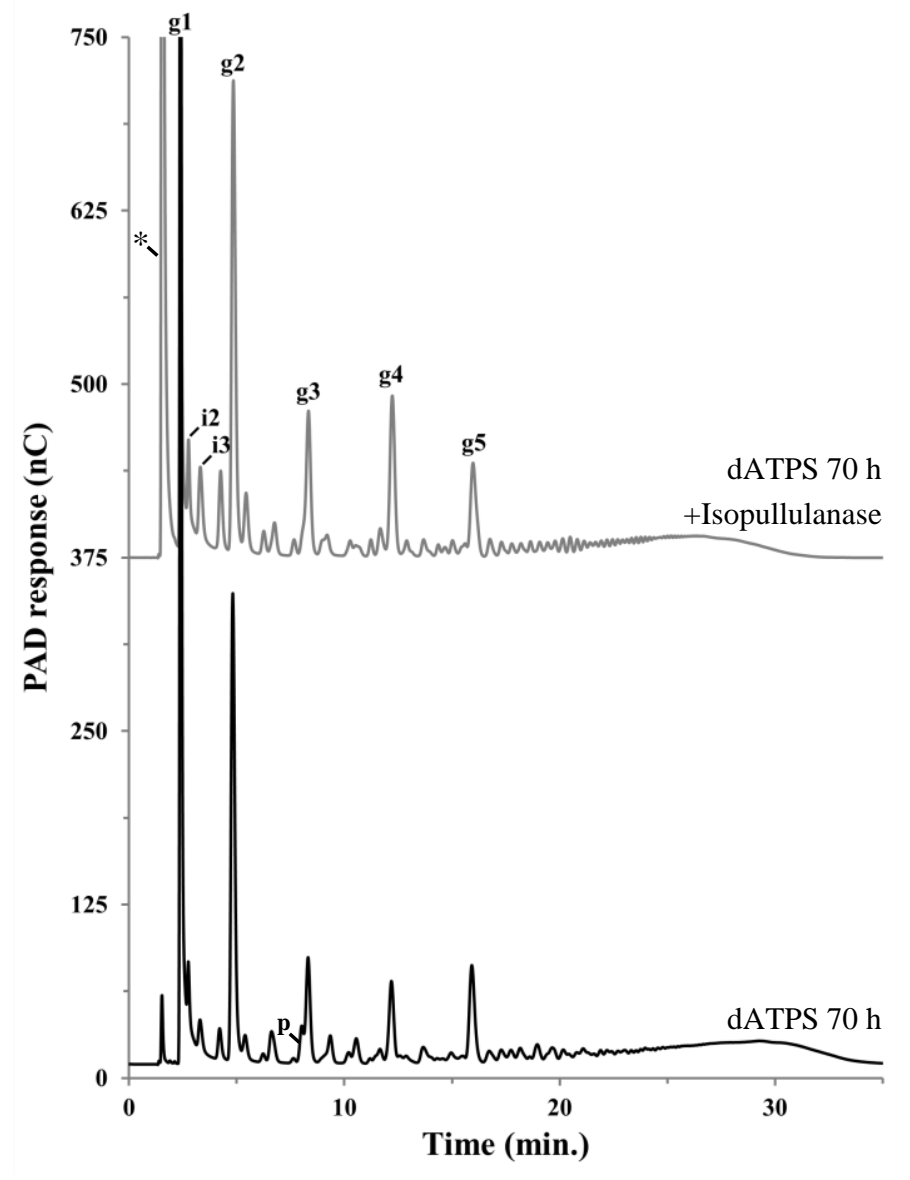

Figure 5. HPAEC profile of debranched amylomaltase treated potato starch (dATPS) after 70 hours of incubation with the GTFB- $\triangle \mathrm{N}$ enzyme (dATPS $70 \mathrm{~h}, \mathbf{C}$ ), fingerprinted with isopullulanase (dATPS $70 \mathrm{~h}+$ Isopullulanase, -). Indicated are; glucose (g1), maltose (g2), maltotriose (g3), maltotetraose (g4), maltopentaose (g5), isomaltose (i2) and isomaltotriose (i3). The elution time of panose (p) was derived from (Dobruchowska et al., 2013) and the glycerol peak (*) from the enzyme solution. 


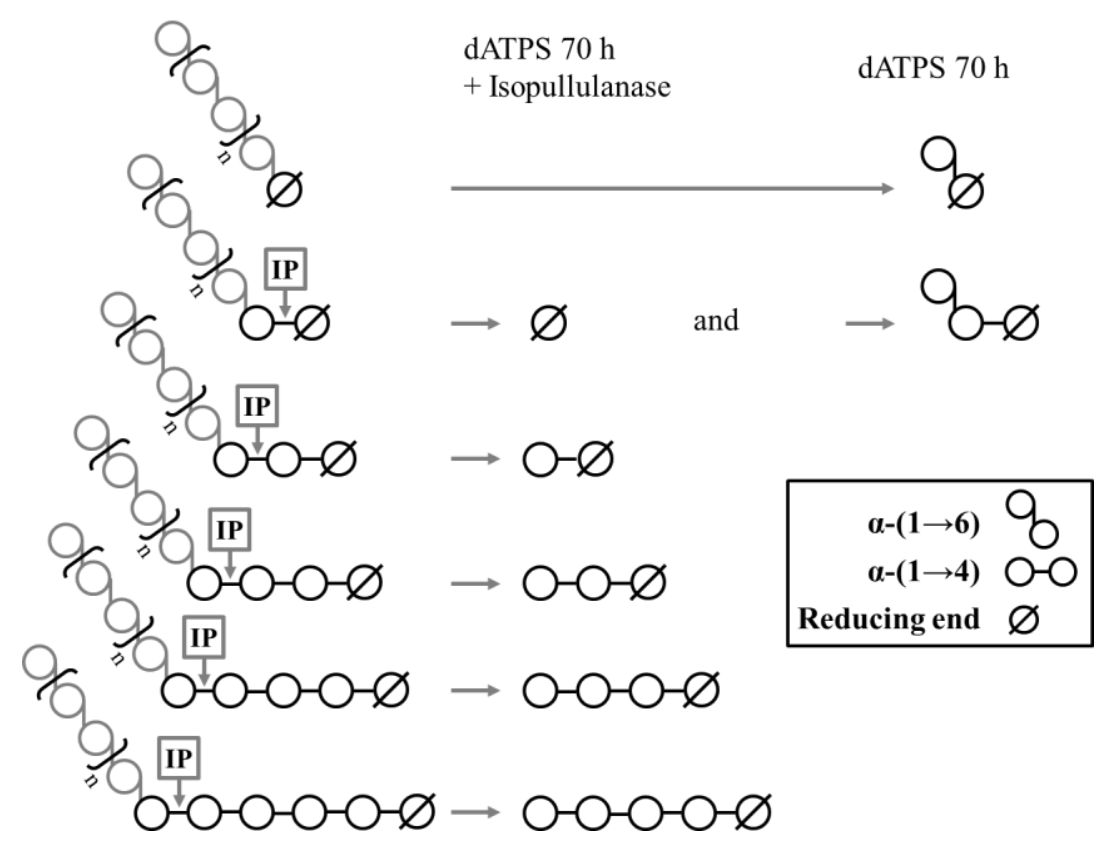

Figure 6. Proposed IMMP structures produced by GTFB- $\Delta N$ activity on linear $\alpha-(1 \rightarrow 4)$ linked glycosidic material. To be proven by enzymatic fingerprinting with isopullulanase or by the presence of certain compounds in the dATPS $70 \mathrm{~h}$ sample.

\subsection{Influence of GTFB- $\triangle N$ on IMMP structure}

Comparing dATPS and dWPS over time (Figure 3 ) shows that a substrate without initial $\mathrm{DP}<6$ material results in less and slower GTFB- $\Delta$ N transferase activity. The GTFB- $\Delta \mathrm{N}$ transferase activity in the dWPS only increases after GTFB- $\Delta$ N hydrolytic activity created sufficient DP $<6$ material. Enzymatic fingerprinting of dATPS after GTFB- $\Delta$ N incubation reveals, surprisingly, no acceptors DP $>6$ at all. Indicating that all material in the final product is either high DP $\alpha-(1 \rightarrow 6)$ linked glycosidic material linked to $\alpha-(1 \rightarrow 4)$ linked glycosidic acceptors ranging from DP 1 to DP 6, as seen in Figure 6, or small oligosaccharides ranging from DP 1 to DP 5 . The addition of small di- or oligosaccharide acceptors could potentially increase the speed and efficiency of GTFB- $\Delta$ N transferase activity leading to less hydrolysis and higher total $\alpha-(1 \rightarrow 6)$ glycosidic linkages contents. Altogether, we can conclude that the presence of $\alpha-(1 \rightarrow 4)$ linked glycosidic acceptors $(\mathrm{DP}<6)$ plays a decisive role for the formation of $\alpha-(1 \rightarrow 6)$ glycosidic linkages during IMMP synthesis. 


\section{Conclusions}

In this study we found that the GTFB- $\Delta$ N elongation of $\alpha-(1 \rightarrow 6)$ linked glycosidic chains goes much further than reported previously, increasing the molecular weight of the largest ATPS fraction from $5.8 \mathrm{kDa}$ to $9.5 \mathrm{kDa}$ and the largest WPS fraction from $3.2 \mathrm{kDa}$ to $6.8 \mathrm{kDa}$ after GTFB- $\Delta \mathrm{N}$ modification. This increase in size is quite remarkable since the GTFB- $\Delta$ N has to completely breakdown the $\alpha-(1 \rightarrow 4)$ linked glycosidic chains in the donor substrate in order to produce the newly introduced $\alpha$ $(1 \rightarrow 6)$ linked glycosidic linkages. Enzymatic fingerprinting of the dATPS substrate after 70 hours of GTFB- $\Delta$ N incubation even revealed $\alpha-(1 \rightarrow 6)$ linked glycosidic chains up to $21 \mathrm{kDa}$. The presence of oligomer acceptors $(\mathrm{DP}<6)$ in the debranched ATPS substrate resulted in a higher final $\alpha-(1 \rightarrow 6)$ glycosidic linkage content and a larger average molecular weight after GTFB- $\Delta \mathrm{N}$ incubation $(69 \% \alpha-(1 \rightarrow 6), 9.5 \mathrm{kDa})$, compared to the debranched WPS substrate $(63 \% \alpha-(1 \rightarrow 6), 6.8 \mathrm{kDa})$. Since no linear $\alpha$ $(1 \rightarrow 4)$ glycosidic linked material larger than DP 6 was detected after 70 hours of incubation, we can conclude that GTFB- $\Delta \mathrm{N}$ is not able to use linear $\alpha-(1 \rightarrow 4)$ linked glucose moieties smaller than DP 6 as a donor substrate. Glucose, maltose, maltotriose, maltotetraose and maltopentaose were confirmed to be acceptors for GTFB- $\Delta \mathrm{N}$ transferase activity, with maltotriose, maltotetraose and maltopentaose being the most preferred. The presence of $\alpha-(1 \rightarrow 4)$ linked glycosidic acceptors $(\mathrm{DP}<6)$ was found to have a big influence on GTFB- $\Delta$ N activity, reducing GTFB- $\Delta$ N hydrolytic activity and increasing GTFB $-\Delta \mathrm{N}$ transferase activity. The use of acceptors during GTFB- $\Delta \mathrm{N}$ incubation will therefore be of great interest for the directed modification of IMMPs in the future.

\section{Acknowledgements}

This project (TKI-2013-B) is jointly funded by AVEBE and TKI as coordinated by the Carbohydrate Competence Center (CCC-ABC; www.cccresearch.nl). The authors thank Pieter de Waard (Wageningen NMR centre) and Margaret Bosveld (Laboratory of Food Chemistry, Wageningen University \& Research) for help and support with the analysis. 


\section{References}

Ayudhaya, P., Pongsawasdi, P., Laohasongkram, K., \& Chaiwanichsiri, S. (2016). Properties of Cassava Starch Modified by Amylomaltase from Corynebacterium glutamicum. Journal of Food Science, 81(6), 1363-1369. https://doi.org/10.1111/1750-3841.13305

Bai, Y., Gangoiti, J., Dijkstra, B. W., Dijkhuizen, L., \& Pijning, T. (2016). Crystal structure of 4,6- $\alpha-$ glucanotransferase supports diet-driven evolution of GH70 enzymes from $\alpha$-amylases in oral bacteria. Structure, 1-12. https://doi.org/10.1016/j.str.2016.11.023

Bai, Y., van der Kaaij, R. M., Leemhuis, H., Pijning, T., Leeuwen, S. S. van, Jin, Z., \& Dijkhuizen, L. (2015). Biochemical characterization of the Lactobacillus reuteri glycoside hydrolase family 70 GTFB type of 4,6- $\alpha$-glucanotransferase enzymes that synthesize soluble dietary starch fibers. Applied and Environmental Microbiology, 81(20), 7223-7232. https://doi.org/10.1128/AEM.01860-15

Dobruchowska, J. M., Gerwig, G. J., Kralj, S., Grijpstra, P., Leemhuis, H., Dijkhuizen, L., \& Kamerling, J. P. (2012). Structural characterization of linear isomalto-/malto-oligomer products synthesized by the novel GTFB 4,6- $\alpha$-glucanotransferase enzyme from Lactobacillus reuteri 121. Glycobiology, 22(4), 517-528. https://doi.org/10.1093/glycob/cwr167

Dobruchowska, J. M., Meng, X., Leemhuis, H., Gerwig, G. J., Dijkhuizen, L., \& Kamerling, J. P. (2013). Gluco-oligomers initially formed by the reuteransucrase enzyme of Lactobacillus reuteri 121 incubated with sucrose and malto-oligosaccharides. Glycobiology, 23(9), 1084-1096. https://doi.org/10.1093/glycob/cwt048

Gu, F., Borewicz, K., Richter, B., van der Zaal, P. H., Smidt, H., Buwalda, P. L., \& Schols, H. A. (2018). In vitro fermentation behaviour of isomalto/malto-polysaccharides using human faecal inoculum indicates prebiotic potential. Molecular Nutrition \& Food Research. https://doi.org/10.1002/mnfr.201601015

Leemhuis, H., Dobruchowska, J. M., Ebbelaar, M., Faber, F., Buwalda, P. L., Van Der Maarel, M. J. E. C., ... Dijkhuizen, L. (2014). Isomalto/malto-polysaccharide, a novel soluble dietary fiber made via enzymatic conversion of starch. Journal of Agricultural and Food Chemistry, 62(49), 12034-12044. https://doi.org/10.1021/jf503970a

Pérez, S., \& Bertoft, E. (2010). The molecular structures of starch components and their contribution to the architecture of starch granules: A comprehensive review. Starch - Stärke, 62. https://doi.org/10.1002/star.201000013

van der Maarel, M., Capron, I., Euverink, G. W., Bos, H., Kaper, T., Binnema, D. J., \& Steeneken, P. (2005). A novel thermoreversible gelling product made by enzymatic modification of starch, 57(10), 465-472. https://doi.org/10.1002/star.200500409

van der Zaal, P. H., Schols, H. A., Bitter, J. H., \& Buwalda, P. L. (2017). Isomalto/maltopolysaccharide structure in relation to the structural properties of starch substrates. Carbohydrate Polymers, 185(November 2017), 179-186. https://doi.org/10.1016/j.carbpol.2017.11.072

van Leeuwen, S. S., Kralj, S., van Geel-Schutten, I. H., Gerwig, G. J., Dijkhuizen, L., \& Kamerling, J. P. (2008). Structural analysis of the $\alpha$-d-glucan (EPS180) produced by the Lactobacillus reuteri strain 180 glucansucrase GTF180 enzyme. Carbohydrate Research, 343(7), 1237-1250. https://doi.org/10.1016/j.carres.2008.01.042 


\section{Supplementary information}

7.1 HPAEC elution of $\alpha-(1 \rightarrow 4)$ linked and $\alpha-(1 \rightarrow 6)$ linked gluco-oligomers

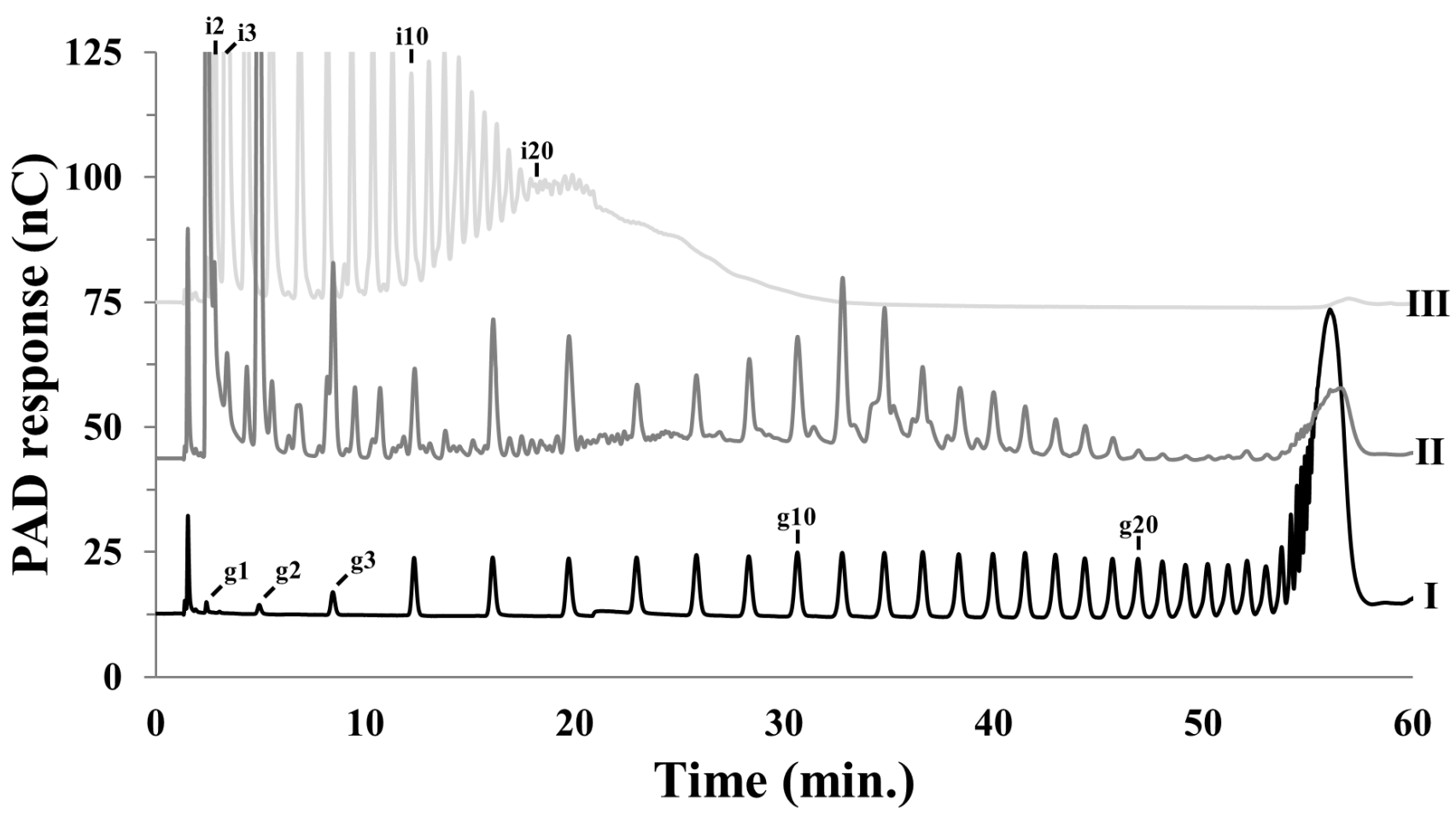

HPAEC profiles of the $\alpha-(1 \rightarrow 4)$ linked glycosidic reference sample (isoamylase debranched amylomaltase treated potato starch (dATPS) (I)), dATPS after $24 \mathrm{~h}$ GTFB- $\Delta$ N treatment (II) and the $\alpha-(1 \rightarrow 6)$ linked glycosidic reference sample (dextranase hydrolysed dextran (III)). Glucose is depicted as $\mathbf{g} \mathbf{1}$ and the degree of polymerisation (DP) of $\alpha-(1 \rightarrow 4)$ linked glucose oligomers is indicated with maltose (g2), maltotriose (g3), $\mathbf{g 1 0}$ and $\mathbf{g 2 0}$ and the DP of $\alpha-(1 \rightarrow 6)$ linked glucose oligomers is indicated with isomaltose (i2), isomaltotriose (i3), $\mathbf{i 1 0}$ and $\mathbf{i 2 0}$. 



\section{Chapter 5}

\section{Directed modification of isomalto/malto-polysaccharides}

Isomalto/malto-polysaccharides are polysaccharides produced by the enzymatic modification of starch-based substrates with 4,6-glucanotransferase (GTFB). In this paper, we demonstrate how the presence of mono- and di-saccharides changes the outcome of GTFB modification. Waxy potato starch was incubated in a one-pot reaction with GTFB and isoamylase including either glucose, maltose or trehalose at two concentrations. The produced IMMPs were analysed with HPSEC, HPAEC, ${ }^{1} \mathrm{H}$ NMR and reducing ends were determined with PAHBAH reagents. It was found that the average molecular weight of the produced IMMPs is negatively correlated to the concentration of added mono- and di-saccharides. The addition of trehalose resulted in a new type of linear non-reducing oligo-/poly-saccharide. Both non-reducing ends of trehalose were found to function as acceptors for GTFB transferase activity. This paper shows that it is possible to control the molecular weight of IMMPs with the addition of mono-/di-saccharides and that the added saccharides are incorporated into the final IMMP.

Based on: van der Zaal, P.H., Bitter J.H. \& Buwalda, P.L. (2018). Directed modification of isomalto/malto-polysaccharides. Carbohydrate Polymers (to be submitted). 


\section{Introduction}

Isomalto/malto-polysaccharides (IMMPs) are polysaccharides produced by the enzymatic modification of starch-based substrates with the 4,6-glucanotransferase (GTFB) enzyme. IMMP structure has been investigated by traditional analysis and by enzymatic fingerprinting (Leemhuis et al., 2014; van der Zaal et al., 2017; van der Zaal et al., 2018). The reaction dynamics of the GTFB enzyme were also investigated and revealed that the GTFB enzyme reacts with starch-based substrates in a substrate acceptor model (van der Zaal et al., 2018). The rate of hydrolysis and the transferase rate of the GTFB enzyme were found to increase in the presence of small glycosidic oligosaccharide material (DP<6) (Bai et al., 2015; van der Zaal et al., 2018). In this paper we will explain how the presence of mono- and di-saccharides influences the outcome of GTFB modification.

The enzymatic modification of $\alpha$-glucans became an established field that shows the potential and flexibility of enzymes in the production of tailor-made polysaccharides (Bissaro, Monsan, Fauré, \& O'Donohue, 2015). Transferase enzymes are particularly suited for controlled and directed modification of $\alpha$-glucans, in this category we can distinguish two main groups: glucansucrases and glucanotransferases. Glucansucrases use sucrose as a substrate and can be used to synthesize a wide variety of $\alpha$-glucan polysaccharides (Leemhuis et al., 2013). Dextransucrases use sucrose to elongate $\alpha$ glucan acceptors with $\alpha-(1 \rightarrow 6)$ linked glycosidic chains (Fang, Wu, \& Xu, 2015; Gan, Zhang, Zhang, \& Hu, 2014; Kothari \& Goyal, 2013) and amylosucrases use sucrose to elongate $\alpha$-glucan acceptors with $\alpha$ - $(1 \rightarrow 4)$ linked glycosidic chains (Kim, Kim, Moon, \& Choi, 2014; Rolland-Sabaté, Colonna, Potocki-Véronèse, Monsan, \& Planchot, 2004). Glucanotransferases are able to transfer single glucoses or partial glycosidic chains from an $\alpha$-glucan donor substrate to an $\alpha$-glucan acceptor molecule. Glucanotransferase activity leads to branching if the reattachment occurs in the middle of an $\alpha$-glucan chain (Grimaud et al., 2013; Suzuki et al., 2015) and leads to cyclization if the reattachment occurs on the same $\alpha$-glucan molecule (Kim et al., 2012; Vongpichayapaiboon et al., 2016; Watanabe et al., 2006). Glucanotransferase activity can also lead to elongation and disproportionation if the reattachment occurs at the end of $\alpha$-glucan chains (Leemhuis et al., 2014; Sorndech et al., 2016).

Combinations of the abovementioned enzymes can be used to make tailor-made $\alpha$ glucan structures such as cyclo-amylose (Kim et al., 2011; Xu et al., 2014), elongated branched amylopectin (Sorndech et al., 2015) and synthetic glycogen (Grimaud et al., 2013; Kajiura, Takata, Kuriki, \& Kitamura, 2010). One of the big challenges for the 
(enzymatic) production of polysaccharides is still the high polydispersity of the substrates and/or products, making it difficult to reliably control the molecular weight of the final polysaccharide. A possible solution could be derived from research on oligosaccharide synthesis with glucansucrases. The synthesis of oligosaccharides with glucansucrases is often directed by altering the ratio between sucrose (substrate) and glycosidic acceptors in the reaction mixture (Iliev et al., 2008; Kothari \& Goyal, 2013; Lee et al., 2008). Although glucansucrases do not use the same substrate, similar principles might also be applicable to the GTFB transferase activity during IMMP synthesis.

Therefore, we investigate the influence of glycosidic acceptors by synthesizing IMMPs in the presence of either glucose, maltose or trehalose. The synthesis of IMMPs was carried out in a one-pot reaction with waxy potato starch, isoamylase, GTFB and the added acceptors. Waxy potato starch in combination with isoamylase form the linear $\alpha$ $(1 \rightarrow 4)$ linked glycosidic chains that function as the substrate for the GTFB enzyme. Previous research has also shown that it is possible to produce IMMPs with high $\alpha$ $(1 \rightarrow 6)$ glycosidic linkage contents in the presence of isoamylase (Leemhuis et al., 2014), but the produced compounds were only analysed with ${ }^{1} \mathrm{H}$ NMR. In this paper we also investigate the molecular weight of produced IMMPs with HPSEC and HPAEC, in order to get more information on the properties of the produced IMMPs and the effect of the added mono-/di-saccharide acceptors. 


\section{Materials \& methods}

\subsection{Materials}

Waxy potato starch (WPS, Eliane 100) was provided by Avebe (Veendam, the Netherlands). Dextran ( $\sim 35 \mathrm{kDa})$ glucose, maltose and trehalose were obtained from Sigma-Aldrich (St. Louis, MO, USA) and isomaltose was obtained from Megazyme (Bray, Wicklow, Ireland). Isoamylase (EC 3.2.1.68) (Pseudomonas sp.) was purchased from Megazyme (Bray, Wicklow, Ireland) and dextranase (EC 3.2.1.11) (Chaetomium erraticum) was obtained from Sigma-Aldrich (St. Louis, MO, USA).

\subsection{GTFB- $\triangle N$ production and activity}

4,6- $\alpha$-glucanotransferase- $\Delta \mathrm{N}$ (GTFB- $\Delta \mathrm{N})$ was produced and purified according to (van der Zaal et al., 2017). GTFB- $\Delta \mathrm{N}$ activity was measured with a GOPOD assay (Megazyme, Bray, Wicklow, Ireland) and was comparable to previous research (Bai et al., 2015; van der Zaal et al., 2017; van der Zaal et al., 2018).

\subsection{IMMP synthesis}

WPS was suspended at $2.5 \%(\mathrm{w} / \mathrm{v})$ in $50 \mathrm{~mL} 20 \mathrm{mM}$ acetate buffer, $\mathrm{pH}=5.0$ containing $5 \mathrm{mM} \mathrm{CaCl}_{2}$. The isoamylase debranched WPS substrate was assumed to have an average DP of 30 (Jane, 1999), acceptors were added in a substrate acceptor ratios of approximately 1:1 and 1:2. For glucose either $47.9 \mathrm{mg}$ (dWPS 1:1 GL) or $90.1 \mathrm{mg}$ (dWPS 1:2 GL) was added, for maltose (monohydrate) either $95.8 \mathrm{mg}$ (dWPS 1:1 MA) or 180.2 mg (dWPS 1:2 MA) was added and for trehalose (di-hydrate) either $100.6 \mathrm{mg}$ (dWPS 1:1 TR) or 189.2 mg (dWPS 1:2 TR) was added. The suspension was autoclaved at $121{ }^{\circ} \mathrm{C}$ for $15 \mathrm{~min}$. and subsequently cooled to $37^{\circ} \mathrm{C}$. IMMP synthesis was carried out by adding $0.3 \mathrm{mg}$ GTFB- $\Delta \mathrm{N} / \mathrm{mg}$ substrate, $0.0267 \mathrm{U}$ isoamylase $/ \mathrm{mg}$ substrate and incubating the solution at $37^{\circ} \mathrm{C}$ for $24 \mathrm{~h}$. After reaction, GTFB- $\Delta \mathrm{N}$ and isoamylase were inactivated by heating the reaction mixture to $95{ }^{\circ} \mathrm{C}$ for $15 \mathrm{~min}$ in a water bath. Next, the solution was cooled to $50{ }^{\circ} \mathrm{C}$, Amberlite MB20-resin (DOW, Midland, MI, USA) was added and the mixture was incubated at $50{ }^{\circ} \mathrm{C}$ for $2 \mathrm{~h}$. The MB20-resin was sieved out. The IMMP solution was stored at $-20^{\circ} \mathrm{C}$ overnight and subsequently freeze-dried.

\section{$2.6{ }^{l} H$ NMR Spectroscopy}

The total $\alpha-(1 \rightarrow 6)$ content was measured with ${ }^{1} \mathrm{H}$ NMR spectroscopy adapted from (van der Zaal et al., 2017). Freeze-dried IMMP was exchanged once with $\mathrm{D}_{2} \mathrm{O}$ by lyophilisation and dissolved in $\mathrm{D}_{2} \mathrm{O}$ (99.9 atom \% D, Sigma-Aldrich, St. Louis, MO, 
USA) at a concentration of $10 \mathrm{mg} / \mathrm{ml}$. Samples were shaken and heated up to $340 \mathrm{~K}$ in a Thermomixer (Eppendorf, Hamburg, Germany) to ensure maximal solubility during the NMR measurement. 1D ${ }^{1} \mathrm{H}$ NMR spectra were recorded at $340 \mathrm{~K}$ on a Bruker Avance 600 spectrometer equipped with a cryo-probe (Billerica, MA, USA) located at the Wageningen NMR Centre. Trehalose was used as a reference for the $\alpha-(1 \rightarrow 1)$ glycosidic linkage, the other linkage types were characterized using the ${ }^{1} \mathrm{H}$ NMR structuralreporter-group concept for $\alpha$-D-glucans (Dobruchowska et al., 2012; van Leeuwen et al., 2008).

\subsection{Determination of molecular weight distribution by HPSEC-RI}

Sample solutions $(2.5 \mathrm{mg} / \mathrm{mL})$ were centrifuged at $7300 \mathrm{x} \mathrm{g}$ and $20{ }^{\circ} \mathrm{C}$ for $10 \mathrm{~min}$ and the supernatant was used for HPSEC analysis. An Ultimate 3000 HPLC (Dionex, Sunnyvale, USA) was used with a column set which consisted of three in series connected TosoHaas (Tokyo, Japan) TSK-Gel columns (4000PWXL-3000PWXL2500PWXL), (6 x $150 \mathrm{~mm}$ ), with a guard column and a Shodex type RI-101 refractive index detector (Showa Denko, K.K., Kawasaki, Japan). With 0.2 M NaNO3 as eluent and a flow of $0.6 \mathrm{~mL} / \mathrm{min}$ at $55^{\circ} \mathrm{C}$. A volume of $10 \mu \mathrm{L}$ sample solution was injected onto the column. A pullulan standard series (180 - 780000 Da) (Fluka) (Buchs, Switzerland) was used for calibration. The buffer and all enzymes were run separately as controls. Data analysis was performed with ChromeleonTM 7.1 software from Thermo Fisher Scientific (Waltham, Massachusetts, USA).

\subsection{Determination of mono- and oligomers by HPAEC-PAD}

Sample solutions were diluted to $0.5 \mathrm{mg} / \mathrm{mL}$ with Millipore water and centrifuged at $7300 \mathrm{x} \mathrm{g}$ and $20{ }^{\circ} \mathrm{C}$ for $10 \mathrm{~min}$, the supernatant was used for HPAEC analysis. The analysis was performed on an ICS5000 High Performance Anion Exchange Chromatography system with Pulsed Amperometric Detection (HPAEC-PAD) (Dionex Corporation, Sunnyvale, USA) equipped with a CarboPac PA-1 column ( 2 x $250 \mathrm{~mm}$ ) and a CarboPac PA-1 guard column ( $2 \times 25 \mathrm{~mm})$. The two mobile phases were (A) 0.1 $\mathrm{M} \mathrm{NaOH}$ and (B) $1 \mathrm{M} \mathrm{NaOAc}$ in $0.1 \mathrm{M} \mathrm{NaOH}$ and the flow rate was set to $0.3 \mathrm{~mL} / \mathrm{min}$. The following gradient was used: 0-50 min 5-40\% B, 50-65 min 40-100\% B, 65-70 min $100 \%$ B ending with a $70-85$ min re-equilibration at $5 \% \mathrm{~B}$. A volume of $10 \mu \mathrm{L}$ sample solution was injected onto the column. Glucose, maltose, isomaltose and trehalose (10$100 \mu \mathrm{g} / \mathrm{mL}$ ) were run as standards. Data analysis was performed with ChromeleonTM 7.1 software Thermo Fisher Scientific (Waltham, Massachusetts, USA). 


\subsection{Reducing ends determination}

The amount of reducing was determined by using a 4-hydroxybenzoic acid hydrazide (PAHBAH) reagents. The deactivated samples were diluted 3 or 4 times to fit the $0-1$ $\mathrm{mg} / \mathrm{mL}$ glucose calibration curve. A solution of $5 \%$ PAHBAH in $0.5 \mathrm{M} \mathrm{HCl}$ was mixed with 4 parts of $0.5 \mathrm{M} \mathrm{NaOH}$. The activated PAHBAH reagents $(200 \mu \mathrm{L})$ was added to $10 \mu \mathrm{L}$ sample in a 96-well plate. The 96-well plate was covered and incubated in a Thermomixer (Thermomixer, Hamburg, Germany) at $70^{\circ} \mathrm{C}$ and $600 \mathrm{rpm}$ for 35 minutes. After cooling to room temperature, the absorbance was measured at $405 \mathrm{~nm}$ with a Tecan Infinite F500 microplate reader (Männedorf, Switzerland). 


\section{Results \& Discussion}

\subsection{IMMP linkage content}

IMMPs with high $\alpha-(1 \rightarrow 6)$ glycosidic linkage contents were produced by incubating waxy potato starch with GTFB- $\Delta \mathrm{N}$ and isoamylase. The glycosidic linkage content of the produced IMMPs was measured with ${ }^{1} \mathrm{H}$ NMR. A typical ${ }^{1} \mathrm{H}$ NMR spectrum for an IMMP with a high $\alpha-(1 \rightarrow 6)$ glycosidic linkage content is displayed in Figure 1. The most relevant peaks are at $5.55 \mathrm{ppm}$ and $5.15 \mathrm{ppm}$ corresponding to the $\alpha-(1 \rightarrow 4)$ and $\alpha$ $(1 \rightarrow 6)$ linked glycosidic linkages respectively. The peaks at $5.42 \mathrm{ppm}$ and $4.82 \mathrm{ppm}$ respectively correspond to the $\alpha$ - and $\beta$-reducing ends. The fact that the reducing ends are visible indicates that the produced IMMPs have a lower average molecular weight than IMMPs produced directly from starch substrates (van der Zaal et al., 2017).

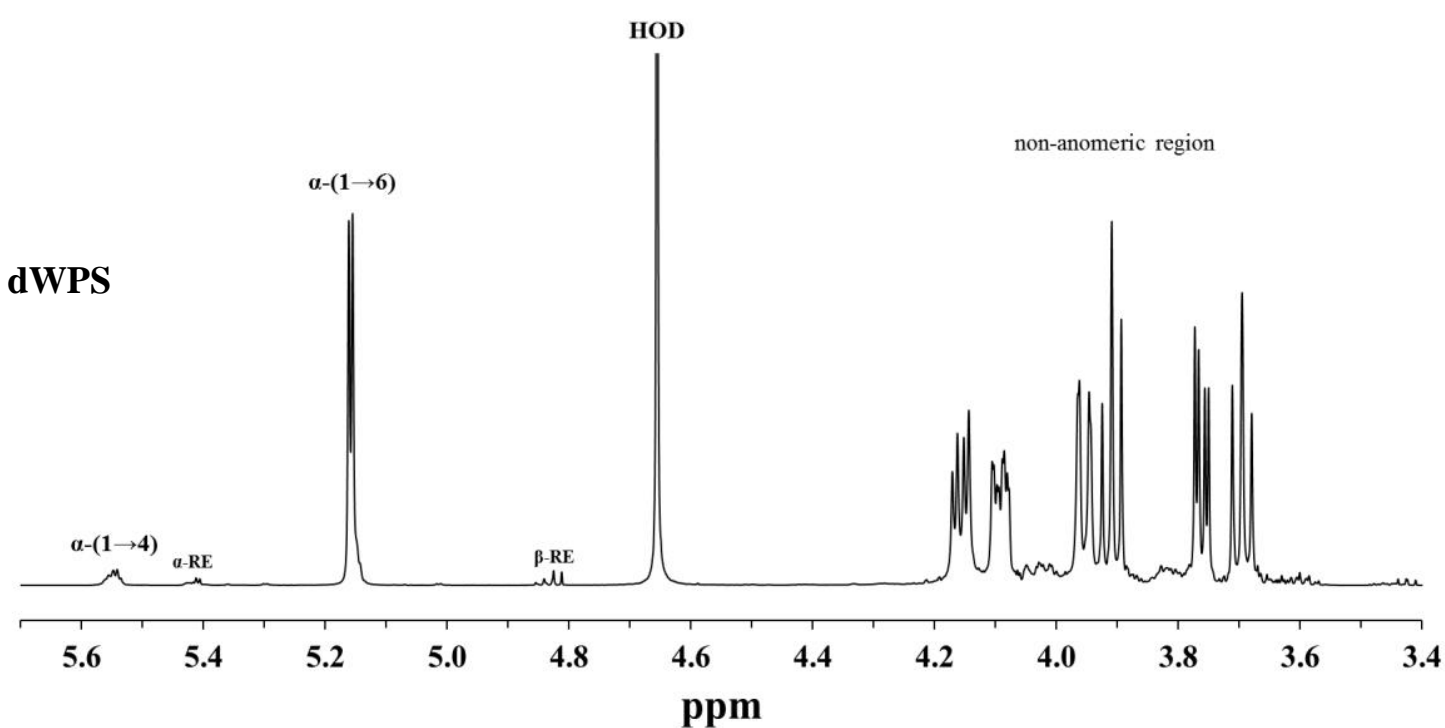

Figure 1. $600 \mathrm{MHz}{ }^{1} \mathrm{H}$ NMR spectra ( $\left.\mathrm{D}_{2} \mathrm{O}, 340 \mathrm{~K}\right)$ of waxy potato starch incubated with GTFB- $\Delta \mathrm{N}$ and isoamylase (dWPS), $\alpha$ - and $\beta$ - reducing ends are indicated with $\alpha-R E$ and $\beta$-RE respectively.

The effect of mono-/di-saccharide acceptors was investigated by adding glucose (GL), maltose (MA) or trehalose (TR) in substrate acceptor ratios of 1:1 or 1:2 as indicated in the sample code (Table 1). The substrate acceptor ratio is a molar ratio based on the average molecular weight of the isoamylase treated waxy potato starch substrate and the molecular weight of the added mono-/di-saccharides. The peak of the $\alpha-(1 \rightarrow 1)$ glycosidic linkage at $5.38 \mathrm{ppm}$ was located with the use of a trehalose reference sample, see below. The obtained linkage contents of all produced IMMPs are compiled in Table 1. 
Table 1. Amount of $\alpha-(1 \rightarrow 6), \alpha-(1 \rightarrow 4)$ and $\alpha-(1 \rightarrow 1)$ linked glycosidic linkages determined with ${ }^{1} \mathrm{H}$ NMR spectroscopy of waxy potato starch incubated with isoamylase and GTFB- $\triangle \mathrm{N}$ (dWPS) in the absence or presence of acceptors. Glucose (GL), maltose (MA) and trehalose (TR) were used as acceptors, added in substrate acceptor ratios of $1: 1$ or $1: 2$.

\begin{tabular}{|l|c|c|c|}
\hline \multirow{2}{*}{ Sample } & \multicolumn{3}{|c|}{${ }^{\mathbf{1}}$ H NMR } \\
\cline { 2 - 4 } & $\mathbf{\%} \boldsymbol{\alpha}-(\mathbf{1} \rightarrow \mathbf{6})$ & $\mathbf{\%} \boldsymbol{\alpha}-(\mathbf{1} \rightarrow \mathbf{4})$ & $\mathbf{\%} \boldsymbol{\alpha}-(\mathbf{1} \rightarrow \mathbf{1})$ \\
\hline dWPS & 91 & 9 & \\
dWPS 1:1 GL & 90 & 10 & \\
dWPS 1:2 GL & 92 & 8 & \\
dWPS 1:1 MA & 91 & 9 & \\
dWPS 1:2 MA & 91 & 9 & \\
dWPS 1:1 TR & 83 & 8 & 9 \\
dWPS 1:2 TR & 77 & 7 & 16 \\
\hline
\end{tabular}

As demonstrated in Table 1, the GTFB- $\Delta \mathrm{N}$ enzyme is able to produce IMMPs with high $\alpha-(1 \rightarrow 6)$ glycosidic linkage contents with the use of isoamylase. The linkage content of the produced IMMPs has more in common with dextran $( \pm 95 \% \alpha-(1 \rightarrow 6))$ (Vettori, Franchetti, \& Contiero, 2012) than with the original waxy potato starch substrate $( \pm 96 \% \alpha-(1 \rightarrow 4))$ (Leemhuis et al., 2014). The $\alpha-(1 \rightarrow 4)$ glycosidic linkage content of the produced IMMPs (7-10\%), shows that most of the waxy potato starch $\alpha-(1 \rightarrow 4)$ linked glycosidic material converted by the GTFB- $\Delta N$ enzyme. The remainder of a small amount of $\alpha-(1 \rightarrow 4)$ linked glycosidic material is in accordance with previous research, which shows that GTFB- $\Delta \mathrm{N}$ transferase activity is limited to $\alpha-(1 \rightarrow 4)$ linked glycosidic material DP $\geq 6$ (van der Zaal et al. (2018)). Meaning that most of the $\alpha-(1 \rightarrow 4)$ linked glycosidic material that is or becomes smaller than DP $=6$ is not available as a substrate for GTFB- $\Delta \mathrm{N}$ transferase activity. The effect of the mono-/di-saccharide acceptors seems to be limited when solely looking at the linkage content measured with ${ }^{1} \mathrm{H}$ NMR.

\subsection{IMMP size distribution}

The proposed influence of acceptors during IMMP synthesis (van der Zaal et al., 2018), becomes apparent when investigating the size distribution of the produced IMMPs with HPSEC and HPAEC (Figure 2). The size distribution of the produced IMMPs is displayed in HPAEC chromatograms (Figure 2, left) and HPSEC chromatograms (Figure 2, right), the black line represents GTFB- $\Delta \mathrm{N}$ and isoamylase treated waxy potato starch in the absence of mono-/di-saccharide acceptors. The position of the glucose, 
maltose and trehalose standards in the HPAEC chromatograms is indicated with G1, G2 and TR respectively and the molecular weights standards (pullulan, 342-107000 Da) are displayed above the HPSEC chromatograms (Figure 2). The 1:1 substrate acceptor ratio is displayed in dark grey and the 1:2 substrate acceptor ratio is displayed in light grey. 

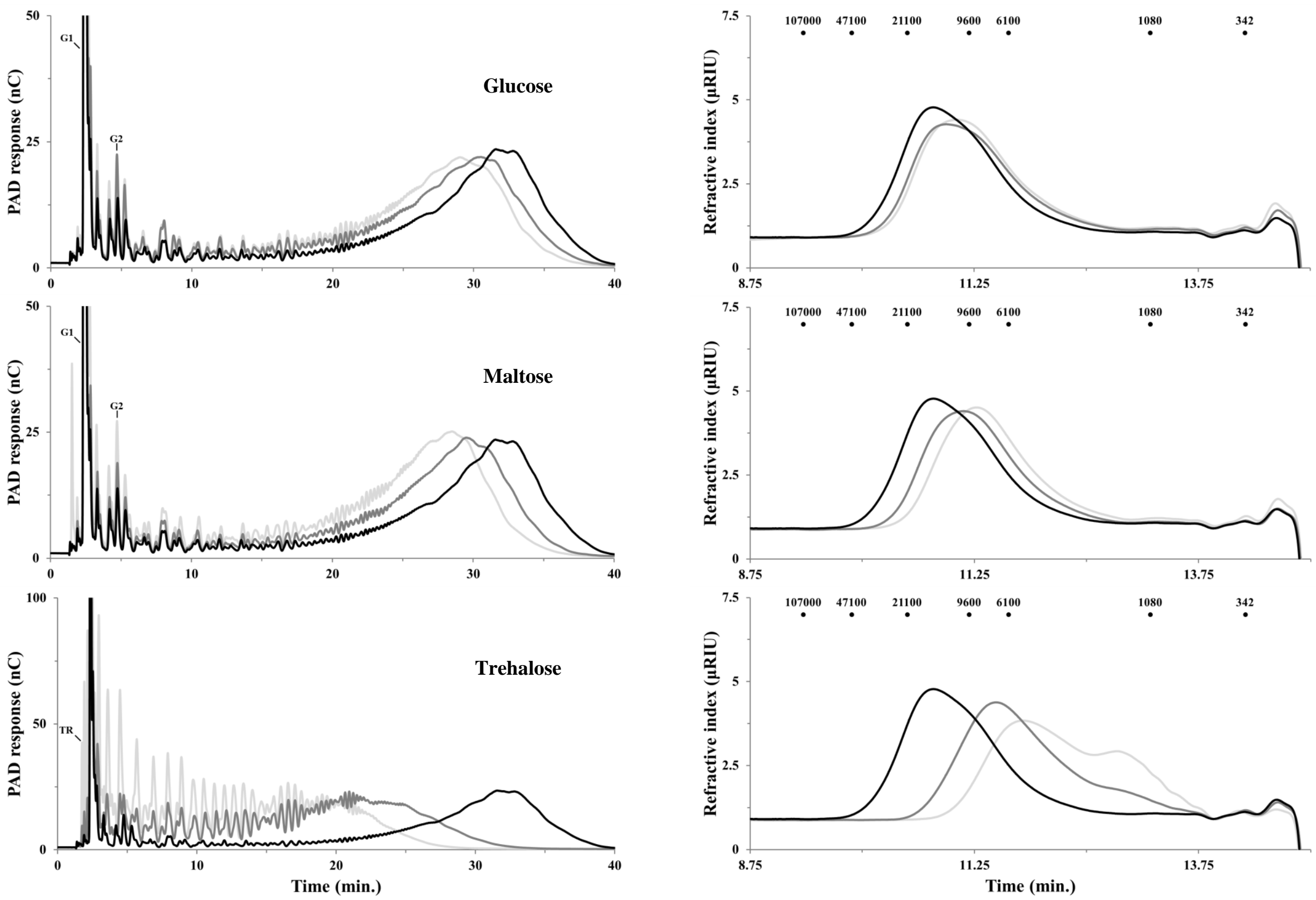

Figure 2. HPAEC (left) and HPSEC (right) profiles of waxy potato starch incubated with GTFB- $\triangle \mathrm{N}$ and isoamylase (dWPS) in the absence or presence of glucose, maltose or trehalose acceptors. Waxy potato starch with isoamylase and GTFB- $\Delta \mathrm{N}$ (black), low concentration of acceptors (1:1, dark grey) and high concentration of acceptors (1:2, light grey). HPSEC pullulan standards are indicated by black dots $(\bullet)$ and are given in Dalton (Da). 
When we investigate the addition of glucose to the reaction mixture, we can observe a shift to the left in the HPAEC chromatograms and a shift to the right in the HPSEC chromatograms (Figure 2), indicating that the addition of glucose causes a slight reduction of average molecular weight of the produced IMMPs. The addition of maltose shows a similar trend but in an amplified way, indicating that maltose is a better acceptor for the GTFB- $\Delta$ N enzyme. This is in accordance with previous research where the addition of maltose shows an increased rate of amylose degradation compared to glucose (Bai et al., 2015). This research shows that it does not only affect the speed of the GTFB$\Delta \mathrm{N}$ hydrolytic activity, but also the size distribution of the product. When more mono/di-saccharide acceptors are added the average molecular weight of the produced IMMP becomes lower, indicating that the GTFB- $\Delta$ N enzyme tends to equally distribute its $\alpha$ $(1 \rightarrow 6)$ glycosidic linkages over the amount of acceptors that are added. This effect can thus be used to effectively control the molecular weight of the produced IMMPs, a feat that is normally very hard to achieve in the production of oligo- and poly-saccharides.

\subsection{Incorporation of trehalose}

Since trehalose is normally not present in GTFB reaction mixtures, we were interested whether the GTFB enzyme is able to incorporate trehalose into a bigger molecule. When we investigate the addition of trehalose, we can observe that trehalose has an even bigger effect on IMMP size distribution (Figure 2). This amplified effect can be explained by the fact that the trehalose molecule contains two non-reducing ends, and both are apparently available for GTFB- $\Delta \mathrm{N}$ transferase activity. The addition of trehalose acceptors in the same molar substrate acceptor ratio as glucose and maltose, effectively doubles the amount of available acceptors for GTFB- $\Delta \mathrm{N}$ transferase activity. The HPAEC chromatograms of the trehalose containing IMMPs also show a shift in peak retention times compared to the IMMP without trehalose (Figure 2 (black line), supplementary information 7.1). This shift is caused by the incorporation of trehalose in the IMMP molecules. Another indication of the incorporation of trehalose in the IMMP structure is the shift of the $\alpha-(1 \rightarrow 1)$ peak in the ${ }^{1} \mathrm{H}$ NMR spectrum of the trehalose containing IMMP compared to pure trehalose (Figure 3). The ${ }^{1} \mathrm{H}$ NMR peak at $5.38 \mathrm{ppm}$ shifts from a doublet for pure trehalose into a broader peak for the IMMP containing trehalose (Figure 3), indicating that there is definitely a change in the structure surrounding the trehalose $\alpha-(1 \rightarrow 1)$ glycosidic linkage. 

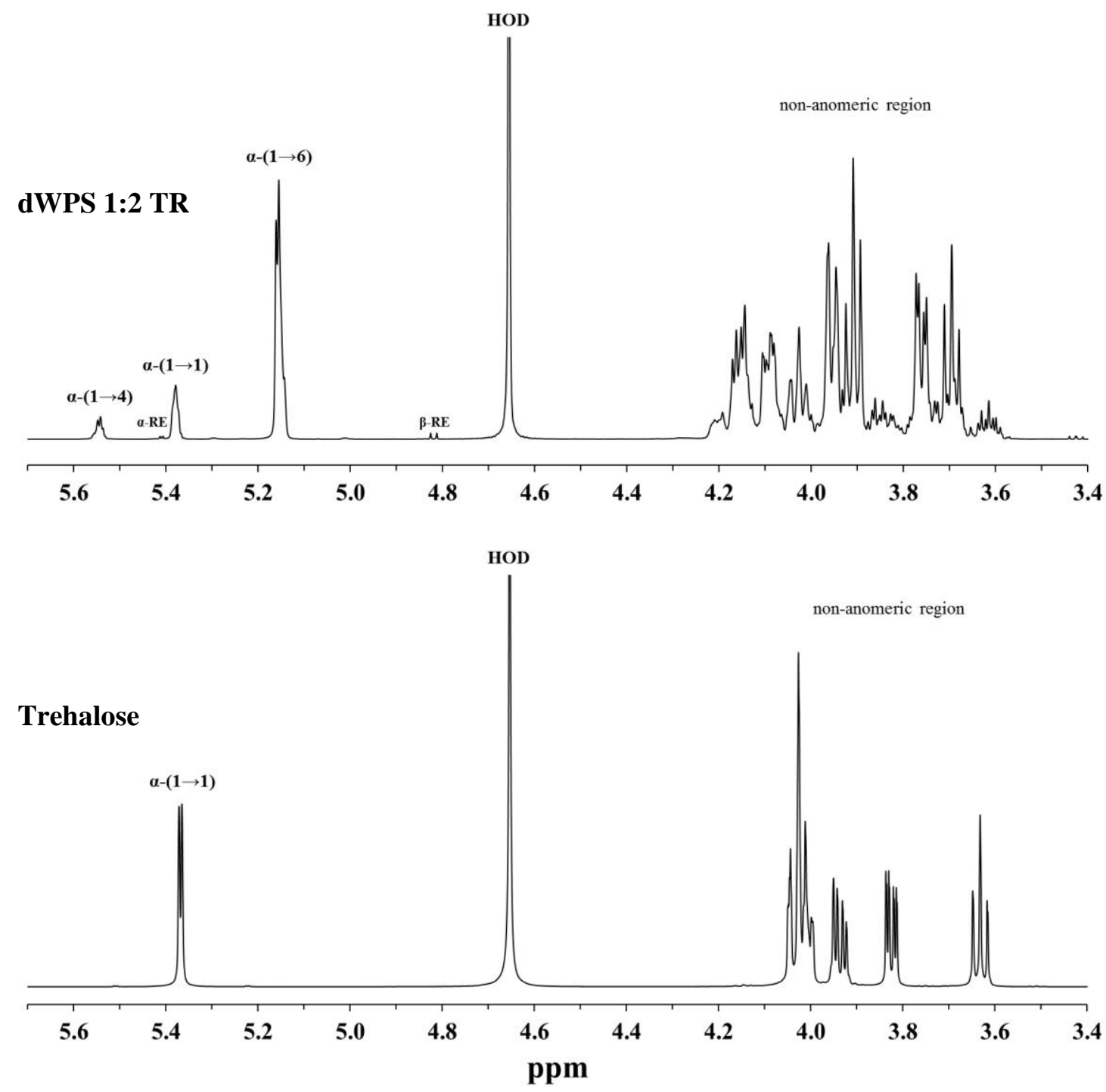

Figure 3. $600 \mathrm{MHz}{ }^{1} \mathrm{H}$ NMR spectra $\left(\mathrm{D}_{2} \mathrm{O}, 340 \mathrm{~K}\right)$ of waxy potato starch incubated with GTFB- $\Delta \mathrm{N}$ and isoamylase (dWPS), waxy potato starch and trehalose incubated with GTFB- $\Delta$ N and isoamylase (dWPS 1:2 TR) and trehalose. The $\alpha$ - and $\beta$ - reducing ends are indicated with $\alpha-\mathrm{RE}$ and $\beta$-RE respectively.

\subsection{Proposed structure of the non-reducing oligosaccharide}

The HPSEC chromatogram of the trehalose containing IMMP with the highest concentration of trehalose acceptors (dWPS 1:2 TR) shows the appearance of two fractions (Figure 3, light grey). These two fractions might consist of two different trehalose containing structures (Figure 4), in which the first structure contains only one non-reducing end of trehalose that is affected by GTFB- $\Delta$ N transferase activity and the second structure where both non-reducing ends are affected by GTFB- $\Delta \mathrm{N}$ transferase activity (Figure 4). Both proposed structures do not contain any reducing ends and could have very interesting applications as one of the first linear non-reducing oligosaccharides. 


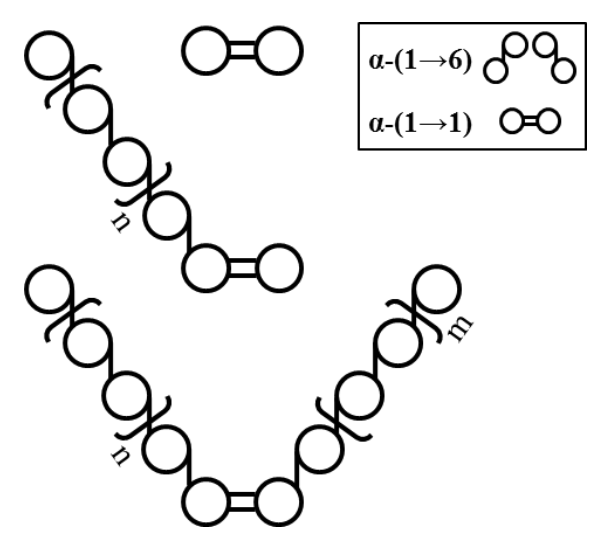

Figure 4. Proposed structure of the non-reducing oligosaccharides formed after incubation of waxy potato starch with isoamylase and GTFB- $\Delta \mathrm{N}$ in the presence of trehalose.

A PAHBAH-assay was used in order to get an indication of the amount of reducing ends present in the produced IMMPs (Figure 5). The addition of glucose and maltose both cause a drop in average molecular weight which understandably results in more reducing ends compared to the larger IMMP produced in the absence of added acceptors (dWPS). The higher substrate acceptor ratios of glucose and maltose (1:2) also show higher amounts of reducing ends in accordance with their smaller size. The trehalose containing IMMPs on the other hand, show a decrease in reducing ends despite the almost doubled decrease in molecular weight compared to the other mono-/di-saccharide acceptors. The fact that the amount of reducing ends did not increase despite the significant smaller size of the trehalose containing IMMPs is another clear indication that trehalose is definitely incorporated in the IMMP structure.

The low amount of reducing ends in trehalose containing IMMPs combined with the natural flexibility of the $\alpha-(1 \rightarrow 6)$ glycosidic linkages, make these non-reducing IMMPs a prime candidate for application as a non-reactive cryo- and/or lyoprotectant (Tonnis et al., 2015). 


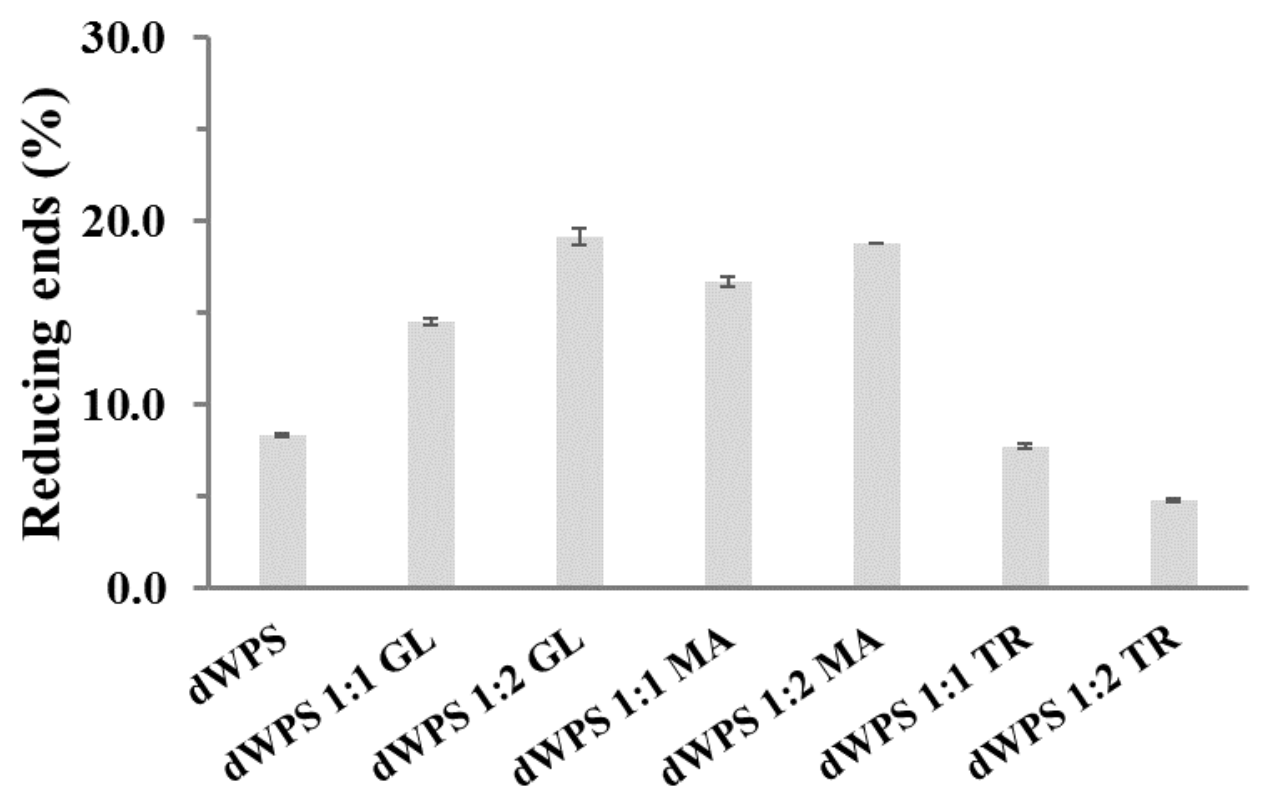

Figure 5. Amount of reducing ends measured with PAHBAH of waxy potato starch incubated with isoamylase and GTFB- $\triangle \mathrm{N}$ (dWPS) in the absence or presence of acceptors. Glucose (GL), maltose (MA) and trehalose (TR) were used as acceptors, added in substrate acceptor ratios of 1:1 or 1:2. 


\section{Conclusions}

This paper is the first to show that the type and amount of added acceptors during IMMP synthesis influences the molecular weight of the final product. The addition of mono/di-saccharide acceptors can thus be used as a tool to synthesize IMMPs of different controlled size ranges. We also demonstrated that trehalose can be incorporated in the IMMP structure, effectively creating one of the first linear non-reducing oligosaccharides. Both non-reducing ends of trehalose proved to be available for GTFB$\Delta \mathrm{N}$ transferase activity, creating a novel non-reducing oligo-/poly-saccharide that is a prime candidate for application as a non-reactive cryo- and/or lyoprotectant.

\section{Acknowledgements}

This project (TKI-2013-B) is jointly funded by AVEBE and TKI as coordinated by the Carbohydrate Competence Center (CCC-ABC; www.cccresearch.nl). The authors thank Pieter de Waard (Wageningen NMR centre) for his help and support with the analysis. 


\section{References}

Bai, Y., van der Kaaij, R. M., Leemhuis, H., Pijning, T., Leeuwen, S. S. van, Jin, Z., \& Dijkhuizen, L. (2015). Biochemical characterization of the Lactobacillus reuteri glycoside hydrolase family 70 GTFB type of 4,6- $\alpha$-glucanotransferase enzymes that synthesize soluble dietary starch fibers. Applied and Environmental Microbiology, 81(20), 7223-7232. https://doi.org/10.1128/AEM.01860-15

Bissaro, B., Monsan, P., Fauré, R., \& O’Donohue, M. J. (2015). Glycosynthesis in a waterworld: new insight into the molecular basis of transglycosylation in retaining glycoside hydrolases. Biochemical Journal, 467(1), 17-35. https://doi.org/10.1042/BJ20141412

Dobruchowska, J. M., Gerwig, G. J., Kralj, S., Grijpstra, P., Leemhuis, H., Dijkhuizen, L., \& Kamerling, J. P. (2012). Structural characterization of linear isomalto-/malto-oligomer products synthesized by the novel GTFB 4,6- $\alpha$-glucanotransferase enzyme from Lactobacillus reuteri 121. Glycobiology, 22(4), 517-528. https://doi.org/10.1093/glycob/cwr167

Fang, Y., Wu, J., \& Xu, Z.-K. (2015). Dextransucrase-catalyzed elongation of polysaccharide brushes with immobilized mono-/di-saccharides as acceptors. Chemical Communications, 51(1), 129132. https://doi.org/10.1039/C4CC06137C

Gan, W., Zhang, H., Zhang, Y., \& Hu, X. (2014). Biosynthesis of oligodextrans with different Mw by synergistic catalysis of dextransucrase and dextranase., 112, 387-395. https://doi.org/10.1016/j.carbpol.2014.06.018

Grimaud, F., Lancelon-Pin, C., Rolland-Sabaté, A., Roussel, X., Laguerre, S., Viksø-Nielsen, A., ... Potocki-Véronèse, G. (2013). In vitro synthesis of hyperbranched $\alpha$-glucans using a biomimetic enzymatic toolbox. Biomacromolecules, 14(2), 438-447. https://doi.org/10.1021/bm301676c

Iliev, I., Vassileva, T., Ignatova, C., Ivanova, I., Haertlé, T., Monsan, P., \& Chobert, J. -M. (2008). Gluco-oligosaccharides synthesized by glucosyltransferases from constitutive mutants of Leuconostoc mesenteroides strain Lm 28, 104(1), 243-250. https://doi.org/10.1111/j.13652672.2007.03555.x

Jane, J. (1999). Effects of amylopectin branch chain-length and amylose content on the gelatinization and pasting properties of starch. Cereal Chemistry, 52(3258), 555. https://doi.org/10.1094/CCHEM.1999.76.5.629

Kajiura, H., Takata, H., Kuriki, T., \& Kitamura, S. (2010). Structure and solution properties of enzymatically synthesized glycogen. Carbohydrate Research, 345(6), 817-824. https://doi.org/10.1016/j.carres.2010.01.013

Kim, B. K., Kim, H. I., Moon, T. W., \& Choi, S. J. (2014). Branch chain elongation by amylosucrase: Production of waxy corn starch with a slow digestion property. Food Chemistry, 152, 113-120. https://doi.org/10.1016/j.foodchem.2013.11.145

Kim, J.-H., Wang, R., Lee, W.-H., Park, C.-S., Lee, S., \& Yoo, S.-H. (2011). \{One-Pot\} Synthesis of Cycloamyloses from Sucrose by Dual Enzyme Treatment: Combined Reaction of Amylosucrase and $\{4-\alpha-$ Glucanotransferase $\}, 59(9), 5044-5051$. https://doi.org/10.1021/jf2002238

Kim, Y.-M. M., Kiso, Y., Muraki, T., Kang, M.-S. S., Nakai, H., Saburi, W., ... Kimura, A. (2012). Novel Dextranase Catalyzing Cycloisomaltooligosaccharide Formation and Identification of Catalytic Amino Acids and Their Functions Using Chemical Rescue Approach. Journal of Biological Chemistry, 287(24), 19927-19935. https://doi.org/10.1074/jbc.M111.339036

Kothari, D., \& Goyal, A. (2013). Structural characterization of enzymatically synthesized dextran and oligosaccharides from Leuconostoc mesenteroides \{NRRL\} B-1426 dextransucrase., 78(10), 
1164-1170. https://doi.org/10.1134/s0006297913100118

Lee, M. S., Cho, S. K., Eom, H. J., Kim, S. Y., Kim, T. J., \& Han, N. S. (2008). Optimized substrate concentrations for production of long-chain isomaltooligosaccharides using dextransucrase of Leuconostoc mesenteroides B-512F. Journal of Microbiology and Biotechnology, 18(6), 11411145. https://doi.org/7442 [pii]

Leemhuis, H., Dobruchowska, J. M., Ebbelaar, M., Faber, F., Buwalda, P. L., Van Der Maarel, M. J. E. C., ... Dijkhuizen, L. (2014). Isomalto/malto-polysaccharide, a novel soluble dietary fiber made via enzymatic conversion of starch. Journal of Agricultural and Food Chemistry, 62(49), 12034-12044. https://doi.org/10.1021/jf503970a

Leemhuis, H., Pijning, T., Dobruchowska, J. M., van Leeuwen, S. S., Kralj, S., Dijkstra, B. W., \& Dijkhuizen, L. (2013). Glucansucrases: three-dimensional structures, reactions, mechanism, $\alpha$ glucan analysis and their implications in biotechnology and food applications. J. Biotechnol., 163(2), 250-272. https://doi.org/10.1016/j.jbiotec.2012.06.037

Rolland-Sabaté, A., Colonna, P., Potocki-Véronèse, G., Monsan, P., \& Planchot, V. (2004). Elongation and insolubilisation of $\alpha$-glucans by the action of Neisseria polysaccharea amylosucrase. Journal of Cereal Science, 40(1), 17-30. https://doi.org/10.1016/j.jcs.2004.04.001

Sorndech, W., Meier, S., Jansson, A. M., Sagnelli, D., Hindsgaul, O., Tongta, S., \& Blennow, A. (2015). Synergistic amylomaltase and branching enzyme catalysis to suppress cassava starch digestibility. Carbohydrate Polymers, 132, 409-418.

https://doi.org/10.1016/j.carbpol.2015.05.084

Sorndech, W., Sagnelli, D., Meier, S., Jansson, A. M., Lee, B., Hamaker, B. R., ... Blennow, A. (2016). Structure of branching enzyme- and amylomaltase modified starch produced from welldefined amylose to amylopectin substrates. Carbohydrate Polymers, 152, 51-61. https://doi.org/10.1016/j.carbpol.2016.06.097

Suzuki, R., Koide, K., Hayashi, M., Suzuki, T., Sawada, T., Ohdan, T., ... Suzuki, E. (2015). Functional characterization of three (GH13) branching enzymes involved in cyanobacterial starch biosynthesis from Cyanobacterium sp. NBRC 102756. Biochimica et Biophysica ActaProteins and Proteomics, 1854(5), 476-484. https://doi.org/10.1016/j.bbapap.2015.02.012

Tonnis, W. F., Mensink, M. A., De Jager, A., Van Der Voort Maarschalk, K., Frijlink, H. W., Hinrichs, W. L. J., ... Hinrichs, W. L. J. (2015). Size and molecular flexibility of sugars determine the storage stability of freeze-dried proteins. Molecular Pharmaceutics, 12(3), 684694. https://doi.org/10.1021/mp500423z

van der Zaal, P. H., Schols, H. A., Bitter, J. H., \& Buwalda, P. L. (2017). Isomalto/maltopolysaccharide structure in relation to the structural properties of starch substrates. Carbohydrate Polymers, 185(November 2017), 179-186. https://doi.org/10.1016/j.carbpol.2017.11.072

van Leeuwen, S. S., Kralj, S., van Geel-Schutten, I. H., Gerwig, G. J., Dijkhuizen, L., \& Kamerling, J. P. (2008). Structural analysis of the $\alpha$-d-glucan (EPS180) produced by the Lactobacillus reuteri strain 180 glucansucrase GTF180 enzyme. Carbohydrate Research, 343(7), 1237-1250. https://doi.org/10.1016/j.carres.2008.01.042

Vettori, M. H. P. B., Franchetti, S. M. M., \& Contiero, J. (2012). Structural characterization of a new dextran with a low degree of branching produced by Leuconostoc mesenteroides FT045B dextransucrase. Carbohydrate Polymers, 88(4), 1440-1444. https://doi.org/10.1016/j.carbpol.2012.02.048

Vongpichayapaiboon, T., Pongsawasdi, P., \& Krusong, K. (2016). Optimization of large-ring cyclodextrin production from starch by amylomaltase from Corynebacterium glutamicum and 
effect of organic solvent on product size., 120(4), 912-920. https://doi.org/10.1111/jam.13087

Watanabe, H., Nishimoto, T., Sonoda, T., Kubota, M., Chaen, H., \& Fukuda, S. (2006). An enzymatically produced novel cyclomaltopentaose cyclized from amylose by an $\alpha-(1 \rightarrow 6)$ linkage, $\{$ cyclo- $\{\rightarrow 6)-\alpha$-d-Glcp- $(1 \rightarrow 4)-\alpha-d-G l c p-(1 \rightarrow 4)-\alpha-d-G l c p-(1 \rightarrow 4)-\alpha-d-G l c p-(1 \rightarrow 4)-\alpha-d-$ Glcp-(1 $\rightarrow\}\}, 341(8), 957-963$. https://doi.org/10.1016/j.carres.2006.02.028

Xu, Y., Zhou, X., Bai, Y., Wang, J., Wu, C., Xu, X., \& Jin, Z. (2014). Cycloamylose production from amylomaize by isoamylase and Thermus aquaticus $4-\alpha$-glucanotransferase. Carbohydrate Polymers, 102, 66-73. https://doi.org/10.1016/j.carbpol.2013.10.065 


\section{Supplementary information}

7.1 The effect of trehalose containing IMMPs on HPAEC elution.

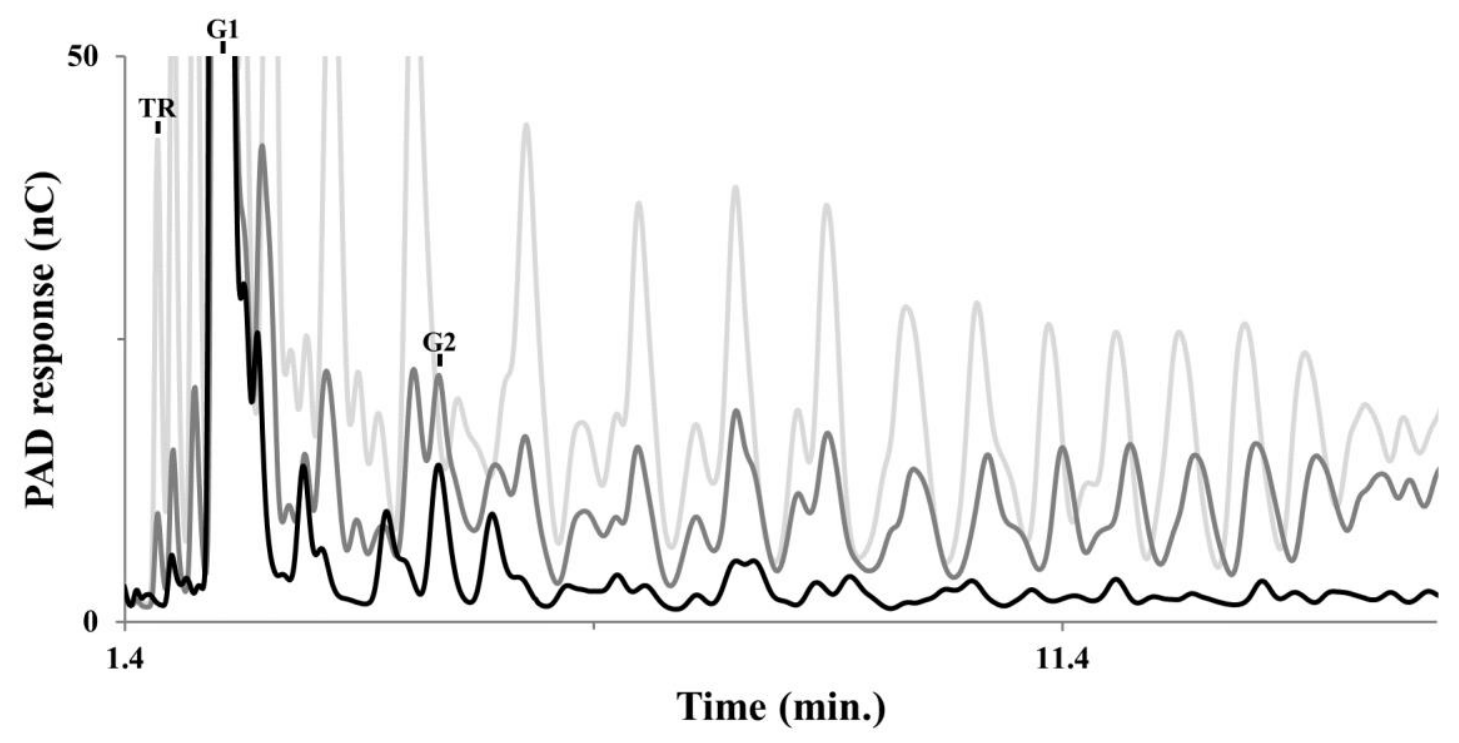

Zoomed in section of the HPAEC chromatogram (Figure 2) of dWPS (black), dWPS 1:1 TR (dark grey) and dWPS 1:2 TR (light grey). The trehalose containing IMMPs (dWPS 1:1 TR, dWPS 1:2 TR) show a notable shift in elution pattern compared to the IMMP without trehalose (dWPS). The shift in specific elution times is caused by the incorporation of trehalose in the IMMP structure and proves that the non-reducing ends of trehalose are susceptible to GTFB- $\Delta$ N transferase activity. 

Chapter 6

General discussion 
In this thesis we investigated the effect of the GTFB enzyme on starch-based substrates and characterized the produced IMMPs. In this chapter we will walk through the findings obtained from this thesis, possible applications for IMMPs and elaborate on the challenges and future perspectives for these novel polysaccharides. The findings were split in two parts, i.e. a) results focussing on the analysis of the structure and composition of analysis of $\alpha$-glucans and b) GTFB substrate interaction although these two parts are interlinked.

\section{Analysis of $\alpha$-glucans}

The analysis of starch and its derivatives has always been challenging due to its insoluble nature. Thus starch granules can be analysed for their crystallinity, size and shape since the techniques needed for that do not need a solution. But revealing the primary structure of starch is more difficult (Pérez \& Bertoft, 2010). In order to fully analyse starch composition it has to be solubilized, however, the solubilisation of starch is not as straight forward as it might seem. The question in starch solubilisation is often: to what extend is the structure of the starch modified by the solubilisation process. Moreover, even after solubilisation, the analysis of starch molecules is still challenging. Therefore it is always important to be transparent on the conditions and methods that are used for the analysis of starch and its derivatives.

Before going into the analysis of structures and enzyme-substrate interaction, in chapter $\mathbf{2}$ we developed and standardized a method for the production IMMPs, in order to ensure that the differences in the produced IMMPs are not a result of poor sample preparation. Autoclavation $\left(121^{\circ} \mathrm{C}, 15 \mathrm{~min}\right.$.) proved to be the best method for simultaneous starch solubilisation and sterilization. The sterilization is an important part of the method since a solubilized starch solution is prone to microbial spoilage at a temperature of $37^{\circ} \mathrm{C}$. The standardized method for IMMP synthesis allows us to equally compare all IMMPs produced in this thesis, creating a solid foundation for the characterization of IMMPs.

Chapter 2 and chapter 3 are both focused on the characterization of IMMPs to facilitate a proper assessment of the impact of GTFB modification on starch substrates. The IMMP characterization in chapter 2 was performed to get a better understanding of the extent of GTFB modification on the different molecular weight fractions of starch substrates. The preparative fractionation of IMMPs into three fractions allowed for some insight in the molecular compositions of the different molecular weight fraction, instead of getting an average analysis over the whole IMMP molecular weight range. This 
method proved to be quite successful, since we could demonstrate that the low and high molecular weight fractions of IMMPs produced directly from starch consist of completely different molecules. The extent of GTFB modification turned out to be much higher for the low molecular weight fraction. This kind of information was used to gain more insight into the preferences of the transferase activity of the GTFB enzyme. The produced IMMPs and their fractions were also analysed with GPC-MALLS (DMSO, $0.05 \mathrm{M} \mathrm{LiBr}$ ), which allowed for better insight in the molecular weight distribution of IMMPs. The characterization of IMMPs in chapter 2 was more extensive than previous research (Yuxiang Bai et al., 2015; Leemhuis et al., 2014), and allowed for a better understanding of the interaction between GTFB and starch substrates.

The enzymatic fingerprinting analysis performed Chapter $\mathbf{3}$ allowed for an even deeper dive in the IMMP structures by revealing and analysing the substructure of IMMPs. While the analysis in chapter 2 allowed for a better insight on the size and linkage composition of IMMPs, it was not able to provide information on specific substructural elements such as; the length of the linear $\alpha-(1 \rightarrow 6)$ linked glycosidic chains introduced by the GTFB enzyme. Since there is no chemical way to specifically hydrolyse IMMPs into their substructural elements, we had to use enzymes that only hydrolyse specific points, such as branching points, in the IMMP molecule. The enzymatic fingerprinting analysis developed for this chapter allowed for a qualitative analysis on the distribution of IMMP substructures. Enzymatic fingerprinting combined with ${ }^{1} \mathrm{H}$ NMR spectroscopy, HPSEC and HPAEC chromatography can replace the more laborious permethylation analysis without losing any structural information. Chapter $\mathbf{3}$ also provided the necessary protocols and structural insight for the 'one-pot' incubations that are performed in chapter 5 .

\section{GTFB substrate interaction}

Chapters $\mathbf{2}$ and $\mathbf{3}$ revealed that the GTFB enzyme shows a general preference for linear substrates. However, the starch substrate used in these chapters often contains multiple fractions and is so complex that it is hard to measure the influence of specific components in the reaction mixture. Therefore we chose to 'simplify' the starch substrate in chapter 4 using isoamylase, in this way, only linear $\alpha-(1 \rightarrow 4)$ linked glycosidic chains were provided as a substrate for the GTFB enzyme. The chain length distribution of the provided substrate proved to have a significant influence on the reaction mechanism of the GTFB enzyme. The presence of small oligosaccharides $(\mathrm{DP}<6)$ turned out to facilitate a transferase reaction, while the absence of these 
oligosaccharides resulted in more hydrolysis. Chapter 4 revealed that the GTFB transferase activity works in a substrate-acceptor model. With the donor substrate being linear $\alpha-(1 \rightarrow 4)$ linked glycosidic chains $(\mathrm{DP} \geq 6)$ and the most preferred acceptors being mono-/di-/oligo-saccharides ( $\mathrm{DP}<6)$. When GTFB is only incubated with the donor substrate of linear $\alpha-(1 \rightarrow 4)$ linked glycosidic chains $(D P \geq 6)$, the GTFB enzyme will show hydrolytic activity creating DP $<6$ acceptors, these DP $<6$ acceptors then, in turn, increase the GTFB transferase activity. This led to the idea that the addition of acceptors could possibly steer the GTFB reaction towards more reliable transferase activity.

Chapter 5 demonstrated that the addition of different types and concentration of mono/di-saccharide acceptors to a 'one-pot' reaction with GTFB, isoamylase and waxy potato starch influences the size of the final product. While the final products still show a relatively high polydispersity index, the average molecular weight is negatively correlated to the concentration of added acceptors. The results in chapter 5 demonstrate that the GTFB enzyme reacts faster with smaller acceptors compared to larger acceptor molecules, resulting in an 'even' distribution of linear $\alpha-(1 \rightarrow 6)$ linkages over the added mono-/di-saccharide acceptors. Chapter 5 also demonstrates that the GTFB enzyme can use trehalose as an acceptor, trehalose shows an increased influence on the size of the final product, since a single trehalose molecule contains two non-reducing ends that function as an acceptor for GTFB transferase activity. The results and methods provided in chapter 5 provide the necessary tools to start using GTFB in a controlled fashion. This will make it easier to direct the modification of IMMPs towards a desired size or functionality. Altogether, this thesis has created a better understanding on IMMP synthesis and provides the necessary tools to analyse and control reactions with the GTFB enzyme.

Now that we have discussed the analysis and the synthesis of IMMPs, we will discuss IMMP properties, the potential applications for IMMPs and the techniques that were developed in this thesis. After that we will elaborate on the future possibilities and challenges that are linked to this research. 


\section{Structural properties of IMMPs}

In order to find suitable applications for IMMPs we first discuss in which way IMMPs are different from their substrate and what their primary structure provides them in terms of structural properties.

The biggest observed difference between the starch substrate and the produced IMMPs is an improved solubility of the IMMPs in all experiments. The improved solubility is even noted in IMMPs that are larger than their substrate (Chapter 4), while larger molecules are usually less soluble. Therefore, we can conclude that the change in structural properties mostly has to do with the linear $\alpha-(1 \rightarrow 6)$ linked glycosidic linkages introduced by the GTFB enzyme. In $\alpha-(1 \rightarrow 4)$ linked glycosidic chains the glucose rings are bound directly to each other, while in $\alpha-(1 \rightarrow 6)$ linked glycosidic chains the glucose rings are separated by an extra carbon (Figure 1).
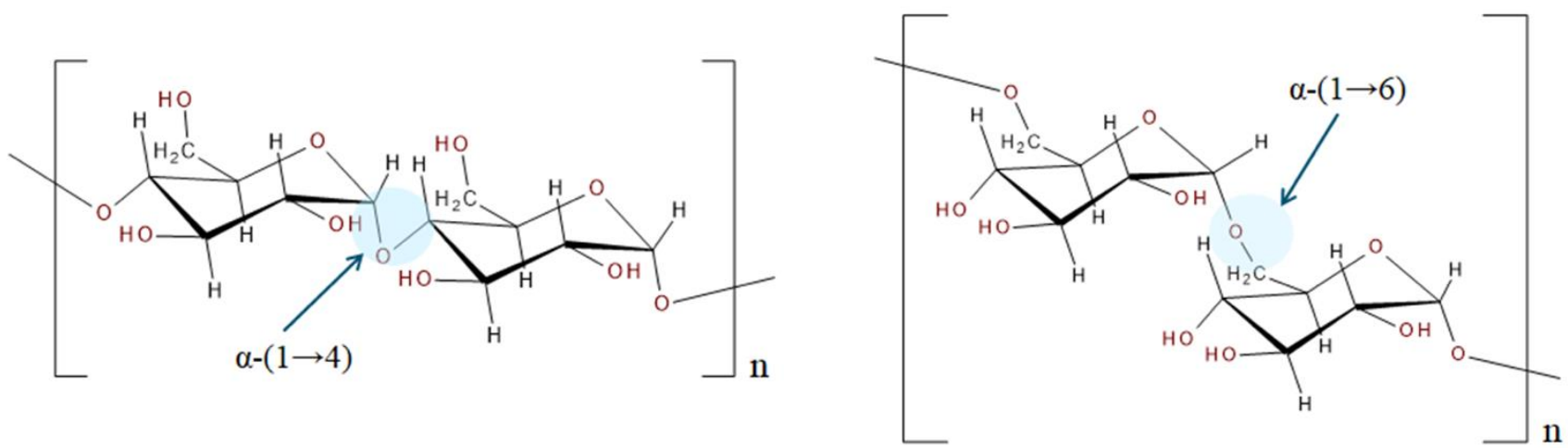

Figure 1. Depiction of a linear $\alpha-(1 \rightarrow 4)$ linked glycosidic linkage (left) and a linear $\alpha-(1 \rightarrow 6)$ linked glycosidic linkage (right).

Since the glucose rings in an $\alpha-(1 \rightarrow 6)$ linked conformation have more space between them there is less intramolecular hydrogen bonding interaction and more hydrogen bonding interaction with the water they are dissolved in, causing better solubility at the cost of their secondary structure. This extra space between the glucose rings also allows for more degrees of freedom giving more flexibility to the $\alpha-(1 \rightarrow 6)$ linked glycosidic chains (Best et al., 2001; Mensink et al., 2015). The proximity of the glucose rings in an $\alpha-(1 \rightarrow 4)$ linked conformation promotes internal hydrogen bonding which causes a secondary helical structure that is fundamental for most of starch's techno-functional properties (Pérez \& Bertoft, 2010). 


\section{Applications of IMMPs}

IMMPs have different potential applications in food and the pharmaceutical industry. First we will cover the potential applications in food, then in the biomedical domain and after that more specifically as a lyoprotectant.

\section{IMMPs in food}

In vitro fermentation with faecal inoculum was performed on several of our IMMPs in a scientific collaboration with the laboratory of Food Chemistry and the laboratory of Microbiology (Wageningen University \& Research) (Gu et al., 2018). The IMMPs produced in this thesis were found to promote several probiotic bacteria indicating a possible prebiotic effect. This research concludes that IMMPs can be classified as slowly digestible food fibres with prebiotic potential and that the IMMPs high in $\alpha-(1 \rightarrow 6)$ linked glycosidic linkages show the most potential to be used as a dietary fibre (Gu et al., 2018; Leemhuis et al., 2014). The Korean dish "kimchi" also contains a majority $\alpha$ $(1 \rightarrow 6)$ linked $\alpha$-glucan (dextran) and this dish is commonly attributed to have positive health effects (Kwak, Cho, Noh, \& Om, 2014; Park, Ahn, Kim, \& Chung, 2013). Although in vitro fermentation with faecal inoculum is not enough to provide any health claims, this research shows that IMMPs have great potential as prebiotic carbohydrates.

In addition to positive health effects, IMMPs also need to show functionality in a food matrix. Although not a lot of research has been done in this area, the majority $\alpha-(1 \rightarrow 6)$ linked dextran has been found to enhance to the sensory properties of acid milk gels (Mende et al., 2013; Tingirikari, Kothari, Shukla, \& Goyal, 2014). Since IMMPs are also high in $\alpha-(1 \rightarrow 6)$ linked glycosidic linkages they might have a similar effect in enhancing the sensory properties of food products.

\section{IMMPs in biomedical applications}

For possible applications of IMMPs rich in $\alpha-(1 \rightarrow 6)$ linked glycosidic linkages we can learn from dextran. Dextran is used successfully in biomedical applications, the most common form contains $95 \% \alpha-(1 \rightarrow 6)$ linked glycosidic linkages and $5 \% \alpha-(1 \rightarrow 3)$ linked glycosidic linkages (Kothari, Das, Patel, \& Goyal, 2015). The amount of $\alpha-(1 \rightarrow 6)$ linked glycosidic linkages is comparable to the IMMPs produced in the presence of isoamylase and GTFB (up to $95 \% \alpha-(1 \rightarrow 6)$ linked glycosidic linkages). Cross-linked dextran, for example, is used as column material for chromatography (Sephadex) and 
clinical grade dextran is used for replacing moderate blood losses by providing colloid osmotic pressure (Heinze, Liebert, Heublein, \& Hornig, 2006). Recent research focusses more on medical hydrogels for accelerated wound healing with dextran as a key component (De Cicco et al., 2014; Ghobril \& Grinstaff, 2015).

The IMMPs that are synthesized with directed modification for controlled molecular weight (Chapter 5), have great potential to be a competitor of dextran in biomedical applications. The main difference between IMMPs and dextran is the substrate from which they are produced. Dextran can either be produced by fermentation or by incubation with a dextransucrase enzyme, both methods however require sucrose as a substrate. Sucrose consists of glucose and fructose, but only the glucose is incorporated in the final dextran molecule, meaning that at best only $50 \%$ of the substrate is utilized for dextran production. The GTFB enzyme, on the other hand, uses a substrate that contains $100 \%$ glucose and therefore has the potential to be much more efficient in the production of $\alpha-(1 \rightarrow 6)$ linked glycosidic chains. The IMMPs produced in this thesis are thus technically more efficient to produce than dextran, making tailor-made IMMPs a suitable competitor for most of dextran's applications.

\section{IMMPs as lyoprotectant}

Lyoprotectants are important for creating the possibility for vaccines to be dried and shipped to remote locations without the need for complete cold chain logistics. Not all proteins are able to withstand drying processes, this is because parts of their structure are supported by the aqueous environment in which they are normally present. When the water is removed from a protein solution, the protein structures that are normally supported by the hydrogen bonds of water can collapse. The function of a lyoprotectant is to protect and stabilize protein by replacing the protein structure supporting hydrogen bonds that water provides with a compound such as a carbohydrate (Tonnis, Mensink, Jager, et al., 2015). In order to properly execute this function, the provided carbohydrate should preferably be flexible in order to reach narrow gaps where the water is supporting the protein structure before drying, but not too small to give additional support (Figure 2, Tonnis et al., 2015). 


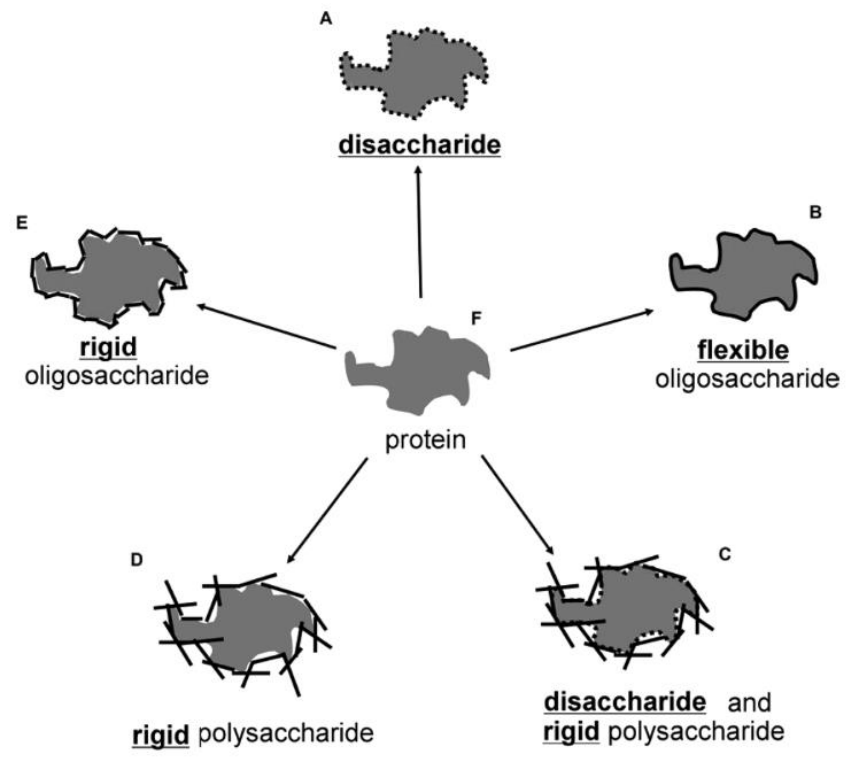

Figure 2. The effectiveness of carbohydrate coating of proteins for lyoprotection depends on the flexibility and size of the used carbohydrate, adapted from Tonnis et al. (2015).

Inulin is an example of an oligo-/poly-saccharide that functions well as a lyoprotectant, since the fructose monomers are not bound ring-to-ring and each ring is separated by a carbon molecule, adding flexibility to its structure (Mensink et al., 2015). The $\alpha-(1 \rightarrow 6)$ glycosidic linkage is doing the same for glucose, separating each consecutive ring with a carbon molecule (Figure 1). However, the presence of reducing ends in a lyoprotectant could result in unwanted destabilization of the protein through the Maillard reaction, since the stabilizing oligosaccharides or polysaccharides are in close contact with the protein. As shown in chapter 5, we are able to produce non-reducing linear oligo-/polysaccharides by synthesizing IMMPs in the presence of trehalose. A flexible nonreducing linear oligo-/poly-saccharide could prove to be a very interesting candidate for delicate medical applications such as the stabilization of proteins during freeze-drying. We recommend that these compounds are checked for their lyoprotective abilities, since the lack of reducing ends enables these carbohydrates to be used in more reactive environments.

\section{Enzymatic fingerprinting on $\alpha$-glucans}

The enzymatic fingerprinting method developed in chapter $\mathbf{3}$ was developed for the analysis of isomalto/malto-polysaccharides, however, the method is also applicable to other $\alpha$-glucans. The enzymatic fingerprinting method uses the activity of several enzymes separately, simultaneously or in successive order to structurally hydrolyse 
IMMPs. Since enzymatic fingerprinting can detect and quantify polysaccharide substructures that are not identifiable with other methods, this method will surely be useful in the polysaccharide field. Especially with the continued discovery of new GTFB-like glucanotransferases that modify $\alpha$-glucans in new unexpected ways, such as the 4,3- $\alpha$-glucanotransferase (GTFC) enzyme that cleaves $\alpha-(1 \rightarrow 4)$ linked glycosidic linkages and introduces $\alpha-(1 \rightarrow 3)$ linked glycosidic linkages (Gangoiti et al., 2017). Will these new glucanotransferases also work in a disproportionating fashion, can the modification with these enzymes also be directed with the addition of mono-/disaccharide acceptors? With all the new carbohydrate active enzymes being discovered yearly, the enzymatic modification of $\alpha$-glucans is just starting (Gangoiti, Pijning, \& Dijkhuizen, 2018), and enzymatic fingerprinting will be a very useful tool to understand these new reaction mechanisms.

\section{GTFB activity on closely related glycosidic acceptors}

As for GTFB, noticing that GTFB is able to glycosylate an $\alpha-(1 \rightarrow 1)$ linked trehalose molecule (Chapter 5), GTFB activity is probably possible on other alternatively linked glucans, such as the $\beta-(1 \rightarrow 4)$ linked cellobiose or the $\beta-(1 \rightarrow 3) / \beta-(1 \rightarrow 6)$ linked $\beta$ glucans. GTFB might also be able to glycosylate aldohexose sugars that are structurally very closely related to glucose, such as mannose, allose and altrose (Figure 3).

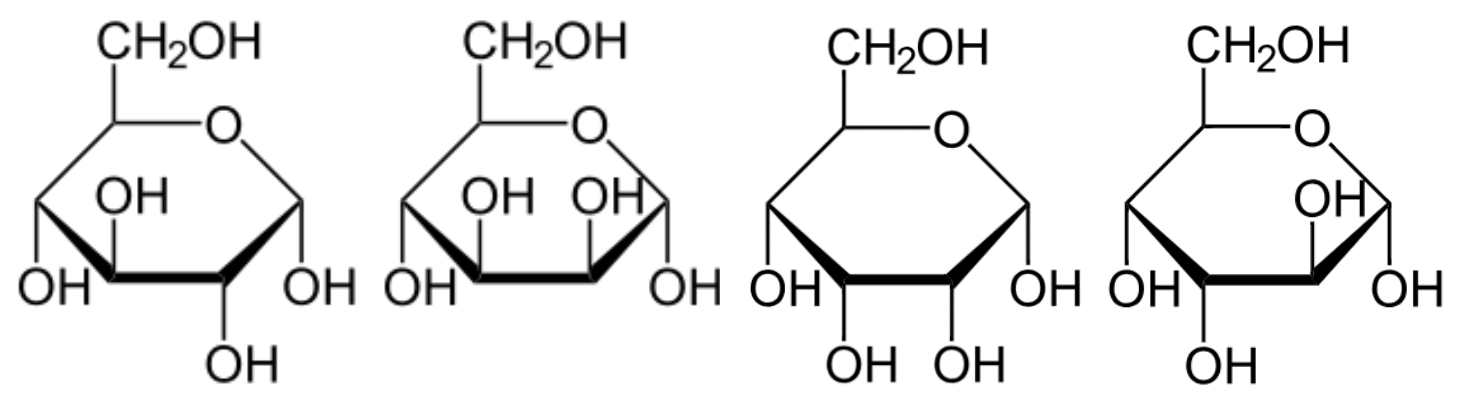

Figure 3. From left to right the chemical structures of glucose, mannose, allose and altrose.

All of the aldohexose sugars shown in Figure 3 have their $\mathrm{OH}$-group on the $\mathrm{C} 4$ position configured in the same direction, this might be enough similarity for GTFB to be able to attach an $\alpha-(1 \rightarrow 6)$ linkage on the closely situated C6 position. Although allose and altrose are rare sugars, mannose is actually frequently occurring in nature in a variety of galactomannans (Srivastava \& Kapoor, 2005). Galactomannans are frequently used in food products (Barak \& Mudgil, 2014), and GTFB might be able to elongate the 
mannose backbone with $\alpha-(1 \rightarrow 6)$ linked glycosidic chains, altering physicochemical properties of these galactomannans. If possible, GTFB might thus be able to functionalize other polysaccharides that either contain glucose or monosaccharides that are structurally closely related to glucose.

\section{GTFB stability}

Some weak points of the GTFB enzyme were also identified during the different experiments in this thesis. Currently there are two main challenges for the GTFB enzyme in order to be applied in an industrial setting. The GTFB enzyme used in this research is very sensitive to shear (Chapter 2), and its optimal temperature at $37{ }^{\circ} \mathrm{C}$ is too low for industrial applications. Experiments in the lab have shown that the GTFB$\Delta \mathrm{N}$ enzyme is particularly sensitive to shear in Erlenmeyer flasks in a standard microbiological incubator. The enzyme did not show any activity on starch when subjected to rotational shear in an Erlenmeyer flask, even at very low rotations per minute. This poses a significant challenge for mass production of IMMPs since the agitation in the process will have to be optimized to minimize starch retrogradation but also prevent inactivation of the GTFB- $\Delta \mathrm{N}$ enzyme. The weakness against shear could possibly originate when the enzyme is interacting with the substrate, the viscosity of the starch solution might also increase the shear forces projected on the enzyme. The enzyme substrate complex might also be particularly sensitive towards shear, since the active centre of the GTFB- $\Delta$ N enzyme has a tunnel were the substrates docks to the enzyme (Bai, Gangoiti, Dijkstra, Dijkhuizen, \& Pijning, 2016). If the substrate is moved harshly while 'docked' in the GTFB- $\Delta$ N enzyme it might be particularly sensitive to shear forces. The other challenge for the GTFB- $\Delta N$ enzyme is thermo-stability, currently the GTFB- $\Delta \mathrm{N}$ enzyme's optimal working temperature is $37^{\circ} \mathrm{C}$. Temperatures above this value will start to denature and inactivate the enzyme. This is not a problem in the laboratory, where we can work in sterile conditions, but working at $37^{\circ} \mathrm{C}$ would be almost impossible to replicate in an industrial environment. The GTFB- $\Delta \mathrm{N}$ enzyme should at least be able to resist temperatures up to $60-70^{\circ} \mathrm{C}$, to avoid microbial spoilage of the highly nutritious starch substrate during the enzymatic modification. The challenge is to create or find a more shear and heat resistant version of the GTFB enzyme, in order to start the industrial production of IMMPs.

When these two major challenges are overcome, the GTFB enzyme would be able to revolutionize the starch industry by creating high value products from a relatively cheap substrate. In biomedical applications, GTFB might be a very suitable competitor for 
dextran and in the food industry the application of IMMPs is focused on health. The addition of a highly soluble dietary fibre in a food product might help the consumer bridge the "fibre gap" without necessarily noticing the presence of the dietary fibre. Isomalto/malto-polysaccharides will hopefully enable the consumer to make healthy food choices without loss in hedonic properties.

Altogether, we can conclude that IMMPs have great potential to disrupt the food and pharma industry. The approach shown in this thesis also fits in the bigger scope of biobased chemistry, which is to use widely available renewable compounds and activate their highest possible purpose. 


\section{References}

Bai, Y., Gangoiti, J., Dijkstra, B. W., Dijkhuizen, L., \& Pijning, T. (2016). Crystal Structure of 4,6- $\alpha-$ Glucanotransferase Supports Diet-Driven Evolution of GH70 Enzymes from $\alpha$-Amylases in Oral Bacteria. Structure, 1-12. https://doi.org/10.1016/j.str.2016.11.023

Bai, Y., van der Kaaij, R. M., Leemhuis, H., Pijning, T., Leeuwen, S. S. van, Jin, Z., \& Dijkhuizen, L. (2015). Biochemical characterization of the Lactobacillus reuteri glycoside hydrolase family 70 GTFB type of 4,6- $\alpha$-glucanotransferase enzymes that synthesize soluble dietary starch fibers. Applied and Environmental Microbiology, 81(20), 7223-7232. https://doi.org/10.1128/AEM.01860-15

Barak, S., \& Mudgil, D. (2014). Locust bean gum: Processing, properties and food applications-A review. International Journal of Biological Macromolecules, 66, 74-80. https://doi.org/10.1016/j.ijbiomac.2014.02.017

Best, R. B., Jackson, G. E., \& Naidoo, K. J. (2001). Molecular Dynamics and NMR Study of the $\alpha-$ $(1 \rightarrow 4)$ and $\alpha-(1 \rightarrow 6)$ Glycosidic Linkages: Maltose and Isomaltose. The Journal of Physical Chemistry B, 105, 4742-4751. https://doi.org/10.1021/jp0040704

De Cicco, F., Reverchon, E., Adami, R., Auriemma, G., Russo, P., Calabrese, E. C., ... Del Gaudio, P. (2014). In situ forming antibacterial dextran blend hydrogel for wound dressing: SAA technology vs. spray drying. Carbohydrate Polymers, 101(1), 1216-1224. https://doi.org/10.1016/j.carbpol.2013.10.067

Gangoiti, J., Pijning, T., \& Dijkhuizen, L. (2018). Biotechnological potential of novel glycoside hydrolase family 70 enzymes synthesizing $\alpha$-glucans from starch and sucrose. Biotechnology Advances, 36(1), 196-207. https://doi.org/10.1016/j.biotechadv.2017.11.001

Gangoiti, J., van Leeuwen, S. S., Gerwig, G. J., Duboux, S., Vafiadi, C., Pijning, T., ... Iacomini, M. (2017). 4,3- $\alpha$-Glucanotransferase, a novel reaction specificity in glycoside hydrolase family 70 and clan GH-H. Scientific Reports, 7(August 2016), 39761. https://doi.org/10.1038/srep39761

Ghobril, C., \& Grinstaff, M. W. (2015). The chemistry and engineering of polymeric hydrogel adhesives for wound closure: a tutorial. Chem. Soc. Rev., 44(7), 1820-1835. https://doi.org/10.1039/C4CS00332B

Gu, F., Borewicz, K., Richter, B., van der Zaal, P. H., Smidt, H., Buwalda, P. L., \& Schols, H. A. (2018). In vitro fermentation behaviour of isomalto/malto-polysaccharides using human faecal inoculum indicates prebiotic potential. Molecular Nutrition \& Food Research. https://doi.org/10.1002/mnfr.201601015

Heinze, T., Liebert, T., Heublein, B., \& Hornig, S. (2006). Functional polymers based on dextran. Advances in Polymer Science, 205(1), 199-291. https://doi.org/10.1007/12_100

Kothari, D., Das, D., Patel, S., \& Goyal, A. (2015). Dextran and food application. In Polysaccharides: Bioactivity and Biotechnology (pp. 735-752). https://doi.org/10.1007/978-3-319-16298-0_66

Kwak, S., Cho, Y., Noh, G., \& Om, A. (2014). Cancer Preventive Potential of Kimchi Lactic Acid Bacteria ( Weissella cibaria, Lactobacillus plantarum ), 19(4), 253-258.

Leemhuis, H., Dobruchowska, J. M., Ebbelaar, M., Faber, F., Buwalda, P. L., Van Der Maarel, M. J. E. C., ... Dijkhuizen, L. (2014). Isomalto/malto-polysaccharide, a novel soluble dietary fiber made via enzymatic conversion of starch. Journal of Agricultural and Food Chemistry, 62(49), 12034-12044. https://doi.org/10.1021/jf503970a

Mende, S., Peter, M., Bartels, K., Dong, T., Rohm, H., \& Jaros, D. (2013). Concentration dependent 
effects of dextran on the physical properties of acid milk gels, 98(2), 1389-1396.

https://doi.org/10.1016/j.carbpol.2013.07.072

Mensink, M. A., Frijlink, H. W., Van Der Voort Maarschalk, K., \& Hinrichs, W. L. J. (2015). Inulin, a flexible oligosaccharide I: Review of its physicochemical characteristics. Carbohydrate Polymers, 130, 405-419. https://doi.org/10.1016/j.carbpol.2015.05.026

Park, J., Ahn, H., Kim, S., \& Chung, C. (2013). Dextran-like Exopolysaccharide-producing Leuconostoc and Weissella from Kimchi and Its Ingredients, 22(4), 1047-1053. https://doi.org/10.1007/s10068-013-0182-x

Pérez, S., \& Bertoft, E. (2010). The molecular structures of starch components and their contribution to the architecture of starch granules: A comprehensive review. Starch - Stärke, 62.

https://doi.org/10.1002/star.201000013

Srivastava, M., \& Kapoor, V. P. (2005). Seed galactomannans: An overview. Chemistry and Biodiversity, 2(3), 295-317. https://doi.org/10.1002/cbdv.200590013

Tingirikari, J. M. R., Kothari, D., Shukla, R., \& Goyal, A. (2014). Structural and biocompatibility properties of dextran from Weissella cibaria JAG8 as food additive. International Journal of Food Sciences and Nutrition, 65(6), 686-691. https://doi.org/10.3109/09637486.2014.917147

Tonnis, W. F., Mensink, M. A., Jager, A. De, Maarschalk, K. V. D. V., Frijlink, H. W., \& Hinrichs, W. L. J. (2015). Size and Molecular Flexibility of Sugars Determine the Storage Stability of Freeze-Dried Proteins. https://doi.org/10.1021/mp500423z 

Summary 
The 4,6- $\alpha$-glucanotransferase (GTFB) enzyme is able to convert starch into isomalto/malto-polysaccharides (IMMPs) and IMMPs have shown potential as soluble food fibres. Soluble food fibres are currently in high demand since the average consumer does not consume enough food fibre in their diets, as discussed in Chapter 1. Although the proof of principle has been demonstrated that IMMPs can be produced from several starches, the influence of the structural properties of the starch substrate and the role of additionally present sugars during IMMP synthesis has remained unknown. Until now, since this has been the core topic of the research presented in this thesis.

In chapter 2, IMMPs were produced from a variety of amylose-rich and amylose-free starches, in order to provide a deeper understanding of IMMP structure in relation to its respective starch substrate. The produced IMMPs were separated into three molecular weight fractions by preparative fractionation with size exclusion chromatography. The different fractions were analysed on their linkage composition and molecular weight profile, using permethylation analysis, ${ }^{1} \mathrm{H}$ NMR spectroscopy and GPC-MALLS. Permethylation analysis was used to distinguish between linear $\alpha-(1 \rightarrow 6)$ linkages introduced by GTFB and starch's native $\alpha-(1 \rightarrow 4,6)$ branching points in all collected fractions. It emerged that the amount of linear $\alpha-(1 \rightarrow 6)$ linkages was consistently higher in IMMP low Mw-fractions and that GTFB activity was limited by native $\alpha-(1 \rightarrow 4,6)$ linkages. The presence of amylose in the starch substrate turned out to be a prerequisite for the incorporation of linear $\alpha-(1 \rightarrow 6)$ linkages in amylopectin.

In order to find out where and to what extend the amylopectin fraction has been modified, and to take a deeper dive in the IMMP structure, we had to develop an enzymatic fingerprinting method, which is presented in chapter 3. Enzymes were used separately, simultaneously or in successive order to specifically degrade and/or reveal IMMP substructures. The enzymatic digests were subsequently analysed with HPSEC and HPAEC to reveal the chain length distribution (CLD) of different IMMP substructures. The enzymatic fingerprinting method revealed that the presence of amylose in the substrate resulted in the formation of linear $\alpha-(1 \rightarrow 6)$ linked glycosidic chains $(13.5 \mathrm{kDa})$ in the former amylopectin fraction. The length of these chains indicates that GTFB transferase activity on amylopectin is more likely to elongate single amylopectin chains than to provide an even spread. Enzymatic fingerprinting also revealed that the GTFB enzyme is capable of introducing large $(20 \mathrm{kDa})$ linear $\alpha-(1 \rightarrow 6)$ linked glycosidic chains in the $\alpha$-glucan substrate.

While the complex starch substrates used in chapter $\mathbf{2}$ and chapter $\mathbf{3}$ revealed some preferences of the GTFB enzyme, the substrates were too complex to reveal the more subtle preferences. In order to get better insight in the reaction dynamics of the GTFB enzyme in chapter 4, it was necessary to 'simplify' its substrate. Two amylose-free starches were incubated with isoamylase in order to create a completely linear substrate. 
GTFB modification of these linear substrates was investigated over time and analysed with ${ }^{1} \mathrm{H}$ NMR, HPSEC, HPAEC combined with GOPOD and PAHBAH assays. The results show that the GTFB modification of linear substrates follows a substrate/acceptor model, in which $\alpha-(1 \rightarrow 4)$ linked glycosidic chains $D P \geq 6$ function as donor substrate, and $\alpha-(1 \rightarrow 4)$ linked glycosidic chains $\mathrm{DP}<6$ function as acceptor. The presence of $\alpha-$ $(1 \rightarrow 4)$ linked $\mathrm{DP}<6$ glycosidic material also resulted in higher GTFB transferase activity, while the absence resulted in higher GTFB hydrolytic activity. The information obtained in chapter 4 provides a better insight into GTFB reaction dynamics and will be useful for substrate selection for the directed modification of IMMPs.

In chapter 5 we demonstrate how the presence of mono- and di-saccharides changes the outcome of GTFB modification. Waxy potato starch was incubated in a one-pot reaction with GTFB and isoamylase including either glucose, maltose or trehalose at two concentrations. The produced IMMPs were analysed with HPSEC, HPAEC, ${ }^{1} \mathrm{H}$ NMR and reducing ends were determined with PAHBAH reagents. It was found that the average molecular weight of the produced IMMPs is negatively correlated to the concentration of added mono- and di-saccharides. The addition of trehalose resulted in a new type of linear non-reducing oligo-/poly-saccharide, in which both non-reducing ends of trehalose were found to function as acceptors for GTFB transferase activity. Chapter 5 shows that it is possible to control the molecular weight of IMMPs by adjusting the concentration of added mono-/di-saccharides, and that these added saccharides are also incorporated in the final IMMP structure.

Chapter 6 discusses the future prospects and potential applications of IMMPs and the methodologies that were developed in this thesis. The enzymatic fingerprinting method and the other characterization strategies developed in this thesis will be of great help for future analysis and modification of $\alpha$-glucans. Altogether, we can conclude that IMMPs have great potential to disrupt the food and pharma industry and since there is no lack in the discovery of new glucanotransferases, the enzymatic modification of $\alpha$-glucans will continue to be a popular field of research. 

Acknowledgements 
I would like to thank my colleagues, family and friends for supporting me during my research and the writing of my thesis. It has been a great undertaking and I could not have done it without you. 
About the author 


\section{Curriculum Vitae}

Pieter Hendrik van der Zaal was born on the $23^{\text {rd }}$ of April 1989 in Leiderdorp, the Netherlands. He grew up in Leiden and finished his secondary education in 2007 (VWO Natuur \& Gezondheid, Da Vinci college). He moved to Wageningen to study Food Technology (Wageningen University \& Research, 2007-2010), with a bachelor thesis in Food Microbiology. Upon completion, he spend one year working and travelling and continued his studies with a Master in Food Ingredient Functionality (Wageningen University \& Research, 2011-2013), a master thesis at the Laboratory of Food Chemistry and an internship at AVEBE (Veendam, the Netherlands). He started his PhD research in October 2013 under the supervision of Dr P.L. Buwalda and Prof. Dr J.H. Bitter at the Biobased Chemistry \& Technology group (Wageningen University \& Research). The results of his $\mathrm{PhD}$ research are presented in this thesis.

Currently, Pieter is working as Global Bio Process Specialist at the Global Innovation and Technology Centre (GITEC) of Anheuser-Busch InBev in Leuven.

Contact:phvanderzaal@gmail.com 


\section{List of publications}

van der Zaal, P.H., Schols, H. A., Bitter J. H. \& Buwalda, P. L. (2017). Isomalto/maltopolysaccharide structure in relation to the structural properties of starch substrates. Carbohydrate Polymers. https://doi.org/10.1016/j.carbpol.2017.11.072

Gu, F., Borewicz, K., Richter, B., van der Zaal, P. H., Smidt, H., Buwalda, P. L., \& Schols, H. A. (2018). In vitro fermentation behaviour of isomalto/malto-polysaccharides using human faecal inoculum indicates prebiotic potential. Molecular Nutrition \& Food Research. https://doi.org/10.1002/mnfr.201601015

Borewicz, K., Hornung, B., Gu, F., van der Zaal, P.H., Schols, H.A., Schaap, P.J. \& Smidt, H. (2018). Isomalto/malto-polysaccharides maintain normal gut functioning while promoting growth and activity of beneficial bacteria. (submitted)

van der Zaal, P.H., Klostermann, C.E., Schols, H. A., Bitter J.H. \& Buwalda, P.L. (2018). Enzymatic fingerprinting of isomalto/malto-polysaccharides. Carbohydrate Polymers. https://doi.org/10.1016/j.carbpol.2018.09.049

van der Zaal, P.H., Klostermann, C.E., Schols, H. A., Bitter J.H. \& Buwalda, P.L. (2018). The influence of linear substrates on 4,6- $\alpha$-glucanotransferase reaction dynamics during isomalto/malto-polysaccharide synthesis. Carbohydrate Polymers (to be submitted).

van der Zaal, P.H., Bitter J.H. \& Buwalda, P.L. (2018). Directed modification of isomalto/malto-polysaccharides. Carbohydrate Polymers (to be submitted). 


\section{Overview of completed training activities}

\section{Discipline specific courses and activities}

Glycosciences (VLAG), Wageningen, the Netherlands, 2014.

Applied biocatalysis (VLAG), Wageningen, the Netherlands, 2014.

Reaction kinetics in food science (VLAG/PDQ), Wageningen, the Netherlands, 2014.

Advanced chemistry (VLAG), Wageningen, the Netherlands, 2016.

CCC annual congress (CCC), Groningen, the Netherlands (2013-2017).

Starch round table EU (SRT), Wageningen, the Netherlands, 2014.

EPNOE congress (EPNOE), Warsaw, Poland, 2015.

Starch round table EU (SRT), Lille, France, 2016.

$68^{\text {th }}$ Starch convention (AGF), Detmold, Germany, 2017.

\section{$\underline{\text { General courses }}$}

Project and time management (WGS), Wageningen, the Netherlands, 2014.

Effective behaviour (WGS), Wageningen, the Netherlands, 2014.

Scientific publishing (WGS), Wageningen, the Netherlands, 2014.

Scientific writing (WGS), Wageningen, the Netherlands, 2015.

Brain training (WGS), Wageningen, the Netherlands, 2015.

Teaching and supervising thesis students (ESD), Wageningen, the Netherlands, 2016.

Career day (WUR), Wageningen, the Netherlands, 2017.

Career assessment (WGS), Wageningen, the Netherlands, 2017.

\section{$\underline{\text { Additional activities }}$}

Preparation of the PhD research proposal.

$\mathrm{PhD}$ trip BCT to China.

Weekly group meetings (2013-2017).

CCC meetings (2013-2017). 

The research described in this thesis (TKI-2013-B) was jointly funded by AVEBE and TKI as coordinated by the Carbohydrate Competence Center (CCC).

Printed by: Digiforce $\|$ ProefschriftMaken

Cover design by Django de Bakker 


\section{Propositions}

1. Enzymes are the future of polysaccharide synthesis and analysis.

(this thesis)

2. The fact that starch is widely available does not mean it is widely understood. (this thesis)

3. Natural variety in substrates should be utilized instead of standardized.

4. Processes based on efficiency are by definition competitive, sustainable processes will therefore claim their place in a profit driven environment.

5. Analysis is never complete, each analysis comes with its own imperfection.

6. Without gut-microbiota our diets would need to be extremely complex.

7. Thinking stands in the way of new ideas.

8. Letting go is the same as starting something new.

Propositions belonging to the thesis, entitled:

"Isomalto/malto-polysaccharides from starch - Reaction pathways and new characterization strategies"

Pieter H. van der Zaal

Wageningen, 14 December 2018 


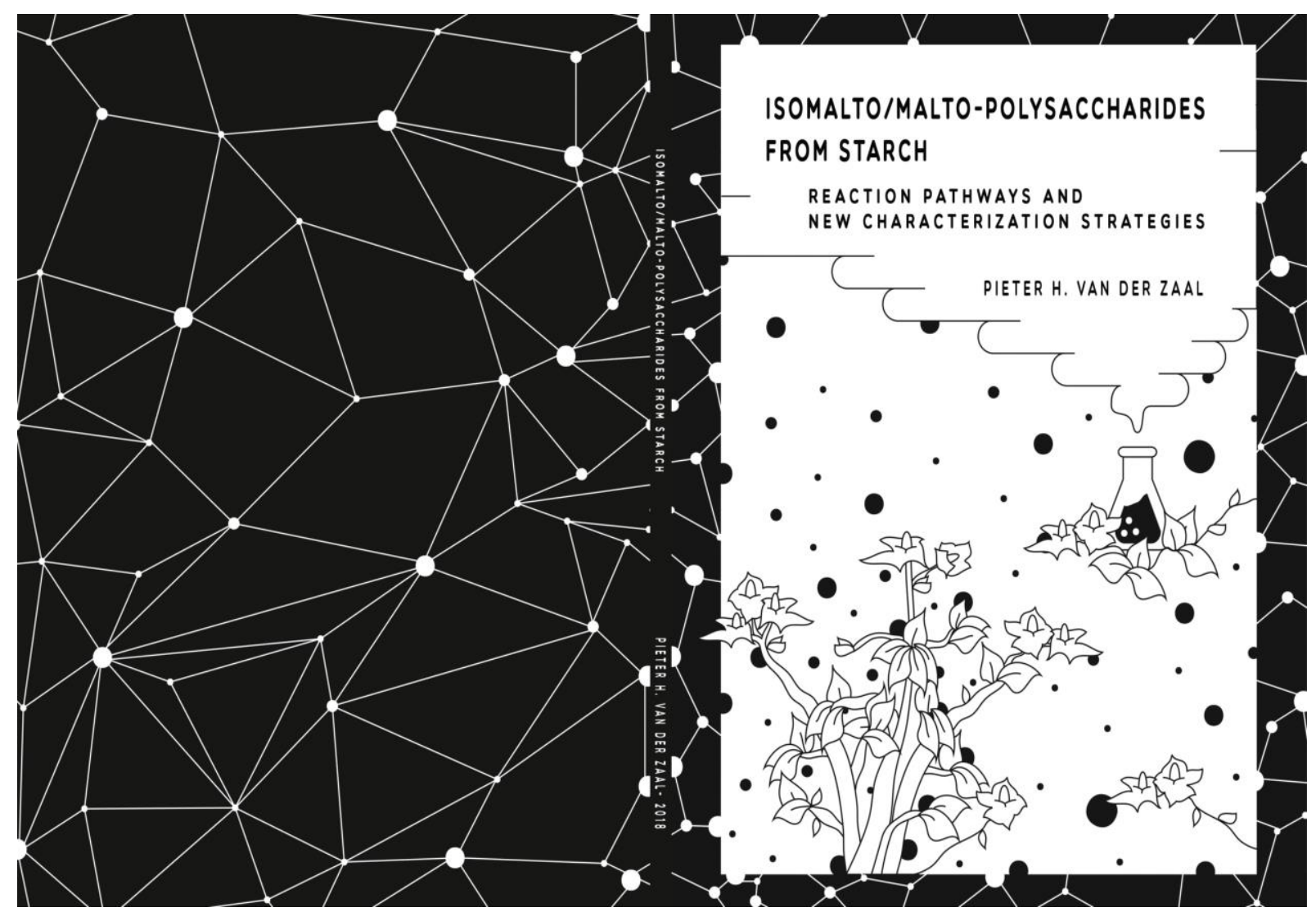

